\title{
Rate Assignment in Wireless Networks: Stability Analysis and Controller Design
}

Seyed Kian Jalaleddini

\author{
A Thesis \\ in \\ The Department \\ of \\ Electrical and Computer Engineering \\ Presented in Partial Fulfillment of the Requirements \\ for the Degree of Master of Applied Science at \\ Concordia University \\ Montréal, Québec, Canada
}

July 2009

(c) Seyed Kian Jalaleddini, 2009 
Library and Archives

Canada

Published Heritage Branch

395 Wellington Street Ottawa ON K1A ON4 Canada
Bibliothèque et

Archives Canada

Direction du

Patrimoine de l'édition

395, rue Wellington

Ottawa ON K1A ON4

Canada
Your file Votre reférence ISBN: 978-0-494-63101-0

Our file Notre référence

ISBN: 978-0-494-63101-0
NOTICE:

The author has granted a nonexclusive license allowing Library and Archives Canada to reproduce, publish, archive, preserve, conserve, communicate to the public by telecommunication or on the Internet, loan, distribute and sell theses worldwide, for commercial or noncommercial purposes, in microform, paper, electronic and/or any other formats.

The author retains copyright ownership and moral rights in this thesis. Neither the thesis nor substantial extracts from it may be printed or otherwise reproduced without the author's permission.
AVIS:

L'auteur a accordé une licence non exclusive permettant à la Bibliothèque et Archives Canada de reproduire, publier, archiver, sauvegarder, conserver, transmettre au public par télécommunication ou par l'Internet, prêter, distribuer et vendre des thèses partout dans le monde, à des fins commerciales ou autres, sur support microforme, papier, électronique et/ou autres formats.

L'auteur conserve la propriété du droit d'auteur et des droits moraux qui protège cette thèse. Ni la thèse ni des extraits substantiels de celle-ci ne doivent être imprimés ou autrement reproduits sans son autorisation.
In compliance with the Canadian Privacy Act some supporting forms may have been removed from this thesis.

While these forms may be included in the document page count, their removal does not represent any loss of content from the thesis.
Conformément à la loi canadienne sur la protection de la vie privée, quelques formulaires secondaires ont été enlevés de cette thèse.

Bien que ces formulaires aient inclus dans la pagination, il n'y aura aucun contenu manquant. 


\section{ABSTRACT \\ Rate Assignment in Wireless Networks: Stability Analysis and Controller \\ Design}

Seyed Kian Jalaleddini

In this thesis, the problem of resource allocation in IS-856 is studied. The problem is first formulated in an interference model framework on reverse channel (uplink). A simple controller is then designed for the system and the closed-loop stability is analyzed using the Lyapunov technique. The possible oscillation in the network output caused by the limit cycles associated with the nonlinear elements in the control loop is analyzed in the framework of describing functions. A dynamic control strategy is developed subsequently for efficient rate assignment in the network. This is carried out in two steps: in the first step, the controller is designed for a simple case when the number of users in the network is fixed and known, and all users are full-buffered. The asymptotic stability property of the proposed controller is verified. Then, the designed controller is further developed for a dynamic network, where the number of active users is subject to change but is known to the users by means of the communication link between the base station and users. In this step, the activation/deactivation of users at different time instants is formulated as a switched system, and sufficient conditions on the speed of activation and deactivation of users are obtained in the control theoretic framework to achieve stability and the desired performance. In the next step, the obtained controller is adjusted properly for the case when the information about the number of active users is not communicated to the users (in order to allocate more bandwidth for data transmission). A controller is also designed to guarantee network stability and performance in the presence of time-delay in the feedback loop. Finally, the long-term fairness in rate allocation is studied. Simulation results are also provided throughout the thesis to elucidate the effectiveness of the proposed approach. 
To my parents

for their love and support 


\section{ACKNOWLEDGEMENTS}

This thesis would not have been possible without the supervision and encouragement of Dr. Amir Aghdam whose guidance and support from the initial to the final level enabled me to develop an understanding of the subject. I would like to thank him for creating a motivating academic environment for his students. I am also grateful to benefit from the constructive comments of Dr. Vahid Tarokh and Dr. Mehdi Alasti on my project throughout my studies at Concordia University.

I would also like to show my gratitude to my friends in Concordia University. I would especially like to thank my friend Kaveh Moezzi, with whom I co-authored two papers throughout my studies. Many thanks also go to Ms. Ranjbaran and Dr. Ahmadreza Momeni for their sincere support and valuable remarks throughout this work. 


\section{TABLE OF CONTENTS}

List of Figures . . . . . . . . . . . . . . . . . . . . viii

List of Abbreviations . . . . . . . . . . . . . . . . . xi

1 Introduction $\quad 1$

1.1 Motivation and Literature Survey . . . . . . . . . . . . . . . 1

1.2 Thesis Outline and Contribution . . . . . . . . . . . 6

2 Background $\quad 9$

2.1 Introduction . . . . . . . . . . . . . . . . . . . 9

2.2 Nonlinear Systems Analysis . . . . . . . . . . . . . . . . . . . 10

2.3 Lyapunov Theory . . . . . . . . . . . . . . . . . . . . . . . . . 12

2.4 Switched Systems . . . . . . . . . . . . . . . . 15

2.5 Describing Function Technique . . . . . . . . . . . . . . . . 16

2.5.1 Prediction of Existence of Limit Cycles . . . . . . . . . . . . . 18

2.6 Quasi-Newton Methods in Optimization . . . . . . . . . . . . . . 19

3 Interference Model for IS-856 Uplink 21

3.1 Introduction . . . . . . . . . . . . . . . . . 21

3.2 Access Terminal . . . . . . . . . . . . . . . . . . 21

3.3 Access Network . . . . . . . . . . . . . . . . . 22

4 Limit Cycle Analysis in Rate Assignment Control Loop 27

4.1 Introduction . . . . . . . . . . . . . . . 27

4.2 Oscillation in Network Output: Limit Cycle Analysis . . . . . . . . . 28

4.3 Simulation Results . . . . . . . . . . . . . . . . . 36

5 Controller Design Using Adaptive Strategy: Stability Analysis 47

5.1 Introduction . . . . . . . . . . . . . . . 47 
5.2 Controller Design for Fixed Number of Users . . . . . . . . . . . . . 48

5.2.1 First-Order Approximation for the Mapping $\tilde{F}(\cdot)$. . . . . . . 52

5.2.2 Second-Order Approximation for the Mapping $\tilde{F}(\cdot) \ldots \ldots$. . . . 54

5.2 .3 Dynamic Rate Selection . . . . . . . . . . . . . 56

5.2.4 Probabilistic Rate Selection . . . . . . . . . . . . 58

5.3 Controller Design for Unknown-fixed Number of Users . . . . . . . . 58

5.4 Controller Design in Presence of Time-Delay . . . . . . . . . . . . . . 61

5.5 Stability Analysis for Varying Number of Users . . . . . . . . . . 66

5.6 Equilibrium Point Adjustment: Long-Term Fairness Study . . . . . . 69

5.7 Simulation Results . . . . . . . . . . . . . . . . 71

6 Conclusions and Future Work $\quad 78$

$\begin{array}{lr}\text { References } & 81\end{array}$ 


\section{List of Figures}

2.1 A nonlinear control system. . . . . . . . . . . . . . . 17

3.1 The mapping $F(\cdot)$ from the feasible rate set $\Gamma$ to the feasible $T 2 P$ set

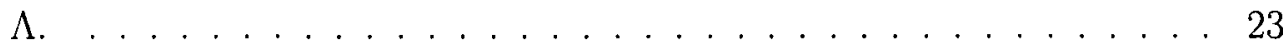

3.2 Rate-control loop in IS-856 uplink, with $n$ active users available in the sector. . . . . . . . . . . . . . . . . 25

3.3 The nonuniform quantizer $\tilde{F}(\cdot)$ as the pragmatic counterpart of the mapping $F(\cdot)$ (solid circles indicate the corresponding feasible set in the mapping $F$ ). . . . . . . . . . . . . 26

4.1 The uplink rate control loop of IS-856. . . . . . . . . . . . . . . . 28

4.2 The root locus trajectories of the IS-856 uplink controller using the discrete-time LTI control law (4.3) . . . . . . . . . . . . . . . 30

4.3 The Nyquist diagram of the uplink rate controller along with the describing function of the nonlinear element of AN . . . . . . . . . 31

4.4 A three-level nonlinear A/D block at the AT . . . . . . . . . . . 33

4.5 Typical input and output signal of a three-level quantizer in the AN. 34

4.6 The describing function of the three-level quantizer with $D=0.1$. . . 36

4.7 The Nyquist diagram of $G(j \omega)$ and $-\frac{1}{N(A, \omega)}$ drawn to identify possible limit cycles in the IS-856 uplink with the control law (4.3) and a threelevel error comparison. . . . . . . . . . . . . . . . . . . . 37

4.8 The step response of the IS-856 uplink using the control law (4.3) with $K=1.23$ and no nonlinear element in the model. . . . . . . . 38

4.9 The step response of the IS-856 uplink using the control law (4.3) when $K=0.1$, and a two-level error comparison. . . . . . . . . . . . . 39 
4.10 The step response of the IS-856 uplink using the linear control law (4.3) with $K=0.01$, and a two-level error comparison. . . . . . . . 39

4.11 The step response of the IS-856 uplink using the optimal values obtained by applying the BFGS method with the initial parameters $z_{0}=0, p_{0}=0$ and $K=0$.

4.12 The step response of the IS-856 uplink, using the optimal values obtained by applying the BFGS method with the initial parameters $z_{0}=-0.1, p_{0}=-0.7$ and $K=0.1 \ldots \ldots \ldots \ldots \ldots \ldots$

4.13 The step response of the IS-856 uplink using the linear control law (4.3) with $K=0.1$, and a three-level error comparison.

4.14 The step response of the IS-856 uplink, which includes a limit cycle as the control gain is greater than $K_{\text {critical }} \ldots \ldots \ldots \ldots \ldots$

4.15 The step response of the IS-856 uplink, using the optimal values obtained by applying the optimal controller given by $z_{0}=-0.0998$, $p_{0}=-0.7001$ and $K=0.111 \ldots \ldots \ldots \ldots \ldots \ldots$

4.16 The step response of the IS-856 uplink, using the optimal values obtained by applying the BFGS method with the initial parameters $z_{0}=0.4781, p_{0}=-0.7990$ and $K=0.2027 \ldots \ldots \ldots \ldots$

5.1 IS-856 uplink rate controller block diagram with the control law (5.1), for the case when the number of users is fixed. . . . . . . . . . . 48

5.2 Linear approximation of the piecewise constant function $\tilde{F}(\cdot)$ as well as two boundary lines containing the function. . . . . . . . . . 53

5.3 A second-order curve as the continuous approximation for the piecewise constant mapping $\tilde{F}(\cdot)$, and second-order curves for its upper and lower-bounds. . . . . . . . . . . . . . . . . 55 
5.4 Magnitude of the error produced when the first-order approximation, second-order approximation, and dynamic allocation method are used for $T 2 P$ in a cell versus number of available active users. . . . . . . . 57

5.5 IS-856 uplink rate controller block diagram with the control low (5.40). 62

5.6 The network output $y(t)$ resulted from using first-order approximation (a) $a=10$; (b) $a=100$. (c) The number of available ATs considered in the simulations at different times. . . . . . . . . . . 73

5.7 The network output $y(t)$ resulted from using second-order approximation (a) $a=10$; (b) $a=100$. (c) The number of available ATs considered in the simulations at different times. . . . . . . . . . . . 74

5.8 The network output $y(t)$ resulted from using dynamic probability rate allocation method (a) $a=10$; (b) $a=100$. (c) The number of available ATs considered in the simulations at different times. . . . . 75

5.9 The output $y_{i}(t)$ resulted from using dynamic probability rate allocation method; (a) the ATs with the control parameters given by (5.70); (b) the ATs with the control parameters given by (5.69). (c) The number of available ATs considered in the simulations at different times. .......................... 77 


\section{List of Abbreviations}

\begin{tabular}{|c|c|}
\hline $\mathrm{A} / \mathrm{D}$ & Analog to Digital \\
\hline $\mathrm{AN}$ & Access Network \\
\hline AT & Access Terminal \\
\hline BFGS & Broyden Fletcher Goldfarb Shanno \\
\hline CDMA & Code Division Multiple Access \\
\hline DF & Describing Function \\
\hline DFP & Davidon Fletcher Powell formula \\
\hline $\mathrm{DRC}$ & Data Rate Channel \\
\hline EV-DO & Evolution Data Optimized \\
\hline HDR & High Data Rate \\
\hline LTI & Linear Time Invariant \\
\hline PDF & Probability Density Function \\
\hline QAM & Quadrature Amplitude Modulation \\
\hline QoS & Quality of Service \\
\hline QPSK & Quadrature Phase Shift Keying \\
\hline $\mathrm{RAB}$ & Reverese Activity Bit \\
\hline RHP & Right Half Plane \\
\hline ROT & Raise Over Thermal \\
\hline SNIR & Signal to Noise plus Interference Ratio \\
\hline SNR & Signal to Noise Ratio \\
\hline TCP & Transmitted Control Proocol \\
\hline TDMA & Time Division Multiple Access \\
\hline $\mathrm{T} 2 \mathrm{P}$ & Traffic to Pilot ratio \\
\hline VT & Video Telephony \\
\hline
\end{tabular}




\section{Chapter 1}

\section{Introduction}

\subsection{Motivation and Literature Survey}

Evolution data optimized (EV-DO, also referred to as EV) is a telecommunication standard for wireless data transmission, typically for broadband Internet access. This standard uses code division multiple access (CDMA) as well as time division multiple access (TDMA) to maximize not only individual user's throughput, but also the total system throughput. In 2000, EV-DO working group was first established in Third Generation Partnership Project 2 (3GPP2), which is a collaborative effort between many standard development organizations (SDO). It was adopted by 3GPP2 as C.S0024, and by Telecommunications Industry Association / Electronic Industries Alliance (TIA/EIA) as IS-856. This technology is compatible with CDMA networks and is optimized for packet data services. It is also used by many mobile phone service providers around the world, particularly the ones that previously employed CDMA networks [1], [7], [2].

EV-DO was initially developed to meet IMT-2000 requirements, i.e., having minimum rate of $2 \mathrm{Mbit} / \mathrm{s}$ in downlink for both the stationary and walking users or $348 \mathrm{kbit} / \mathrm{s}$ for the users in a moving vehicle. Second generation systems, on the 
other hand, provide (only) rates ranging from $9.6 \mathrm{kbit} / \mathrm{s}$ to $28.8 \mathrm{kbit} / \mathrm{s}$. Originally, the standard was called high data rate (HDR), but was later renamed to EV-DO after being ratified by the International Telecommunication Union (ITU) and was given the numerical designation IS-856 [3], [4]. The forward link and reverse link in IS-856 consume $1.25 \mathrm{MHz}$ of spectrum. However, due to multi-user diversity, its forward link supports higher bit rates compared to reverse link [28]. In EV-DO, the channel on forward link is time-multiplexed, which means that a single mobile has full access of the forward traffic channel during a particular time slot, i.e, it receives the full power of the cell transmitter. In particular, at any point in time, each mobile measures signal to noise plus interference ratio (SNIR). Then, the mobile estimates its air-link capacity accordingly [4], as well as the rate that the estimated SNIR can support with a sufficiently error rate. Moreover, the mobiles report their air-link capacity (often called data rate channel (DRC)) to the base station at any time to request the computed rate [28], [4], [29]. By employing this technique, EV-DO is able to modulate each user's time slot independently. Therefore, on one hand, mobiles in favorable $\mathrm{RF}$ condition can benefit from complex modulation techniques, and on the other hand, mobiles in poor RF condition can use simpler and more redundant signals.

In EV-DO Rev.0, the peak data rate that a user can have in the network is achieved by employing 16-QAM modulation which is equal to $2458 \mathrm{kbit} / \mathrm{s}$ in good channel condition with high signal to noise ratio (SNR). Scheduler is the other important component of the EV-DO on downlink channel. The scheduler is designed to maximize the sector throughput while guaranteeing each user a certain quality of service. EV-DO also benefits from employing parallel codes and turbo decoding techniques [1], [7], [4].

On the uplink of EV-DO Rev.0, a pilot signal is included along with the data, which is useful for decoding purposes. The reverse link has both open and closed 
loop power control. In the open loop structures the transmission power is set based upon the received power on the forward link. In the closed loop structure, on the other hand, the uplink power is adjusted up or down 800 times a second, as specified by the serving sector. All the mobiles use code division and transmit signals to the base station using QPSK modulation. The maximum available rate for user data is $153.2 \mathrm{kbit} / \mathrm{s}$. In reality, however, this maximum can rarely be achieved, and the typical rates are between $20-50 \mathrm{kbit} / \mathrm{s}$ [1], [7], [5].

In EV-DO Rev.A, some new features are added to the protocol while keeping it compatible with the EV-DO Rev.0 which allows the system for low-latency communication. In particular, on the downlink several new data rates are added, increasing the maximum data rate to $3072 \mathrm{kbit} / \mathrm{s}$. Also, the ability for sharing the same time slot (multi-user packets) by more than one mobile is incorporated into the system, and the quality of service (QoS) flags are introduced. Moreover, similar to the downlink, some changes are made on uplink to support more complex modulation and high bit rates. A Secondary pilot was added, which is activated by the users in order to achieve enhanced data rates. As a result, the maximum data rate on the uplink was increased to about $1.8 \mathrm{Mbit} / \mathrm{s} \mathrm{[7],} \mathrm{[36].}$

By comparing EV-DO Rev.0 and EV-DO Rev.A, one can observe that most developments were made on the reverse link. In other words, EV-DO Rev.0 was designed particularly for data applications where the volume of data received by the user is greater than the volume transmitted on the uplink. This means that EV-DO Rev.0 provides asymmetric data transfer on the uplink and downlink. While this type of data transfer is utilized in the majority of existing data applications, the new multimedia applications such as interactive wireless gaming, video telephony (VT) and camera phones require a wireless network that is able to provide symmetry on uplink and downlink [36].

The presence of interference in IS-856 uplink is an important issue which is 
caused by the spectral overlap, and needs to be taken into consideration in performance analysis. Practically, in CDMA systems any user that transmits data to the base station is, in fact, a source of interference. In addition, there are other interferences caused by those users that are not in soft hand-off with the base station. This issue is of great importance because in such networks users transmit data at the same time over a prescribed spectrum in a common medium [8], [9], [37]. A reference value for the interference magnitude can be defined based on the ideal expectations. There will be performance implications as the interference increases above this reference level [9]. On the other hand, as the interference level decreases below this level, the problem of under-utilization of the network resources is encountered. The effect of interference in this type of networks is often compensated by transmitting minimal control signaling from the base station to the users (and vice versa), and applying a proper control strategy. In other words, the base station broadcasts a common signal to all users based on its measurement of network interference, and users adjust their rates accordingly. This is a single bit signal which is called the reverse activity bit (RAB). This bit is set to +1 by the base station if the existing interference in the network is below the reference value, and is set to 0 if the interference level is above this threshold [7]. In the context of control, this binary comparison introduces a nonlinearity (on-off element) in the closed-loop system through the control operation.

A simple state transition mechanism is implemented in IS-856 Revision 0 standard, to properly adjust user rates [7], [1], [19]. In this algorithm, each user sets its transmission rate locally by monitoring a common single bit and acting upon it, accordingly (note that it is normally desirable to assign as many bits as possible for data, and as few bits as possible for control signal). Due to its performance deficiency, this mechanism is replaced by a token bucket mechanism in IS-856 Revision A [10], [7]. In [10], [11], [13], [38], [39], using a framework analogous to the one 
presented in [17], [18], the rate control problem is formulated as a utility maximization problem and the fairness and stability issues are studied. In the above works, distributed resource allocation is formulated as a utility maximization problem, analogously to transmitted control protocol (TCP) congestion-control algorithms for the Internet. In other words, the rate allocation problem in the above-mentioned papers is studied in the context of auctioning algorithms subject to existing constraints in the physical system in order to allocate the available resources to all users in a fair manner. In particular, a resource allocation algorithm is proposed in [11], and its long-term and short-term fairness is verified with respect to interference power constraint. Short-term fairness which has been previously studied in the context of contention-based wireless multiple access protocols such as [32] cannot be applied in CDMA where multiple mobiles transmit data simultaneously. In [11], the long-term fairness is investigated based on the results in [30], [31] and [28] which is, in fact, the average-rate guarantee for a particular mobile. Then, the admission control mechanism of the network is then ensures that maintaining this average rate guarantees for all mobiles does not cause the total received interference to exceed a certain threshold.

In [14], a novel framework is proposed to tackle the underlying problem using a control theoretic view. Transmission of the RAB from the base station to the users is incorporated in the forward path of the closed-loop model, and a simple compensator is designed for the network to achieve output regulation. The stability of the resultant closed-loop system is then verified using describing function technique, as the overall system is nonlinear due to the existence of the two-level comparator in the control loop. While this type of stability analysis is quite reliable in general, the result in [14] may not be accurate enough in practice, due to the simplifying assumptions in the network model. 
In this thesis, control techniques are employed to investigate important properties of the network such as stability and output oscillation. A dynamic adaptive control strategy is introduced, and the Stability of the network is analyzed using the Lyapunov technique. The proposed algorithm suffers mainly from two shortcomings: First, the signaling between the base station and users is not minimal in the underlying algorithm. Furthermore, the effect of delay in the control loop is not taken into account in the stability analysis. These shortcomings are addressed later by using minimal control signaling and a more accurate model for the network, in which time-delay is modeled in the forward path of the control loop. A fairness strategy is also provided for a more efficient rate assignment.

\subsection{Thesis Outline and Contribution}

This thesis consists of the results in [22] and [26], as well as some new results concerning oscillation in the network output, and a fairness strategy for a more efficient rate assignment. The papers [22] and [26] are:

[22] K. Jalaleddini, K. Moezzi, A. G. Aghdam, M. Alasti, and V. Tarokh, "Controller design for rate assignment in wireless networks," in Proceedings of IEEE International Conference on Communications, Dresden, Germany, June 2009.

[26] K. Moezzi, K. Jalaleddini, A. G. Aghdam, M. Alasti, and V. Tarokh, "An adaptive rate assignment strategy for CDMA2000 IS-856 subject to RAB delay," in Proceedings of IEEE Global Communications Conference, Honolulu, USA, December 2009.

Furthermore, a complete version of the above papers with some additional results will be submitted for journal publication in August 2009.

As the main contribution of this thesis, powerful control techniques are employed to solve some of the existing problems in wireless communication networks. 
These techniques are used to investigate the presence of oscillation in the network output, design adaptive rate assignment strategies, and derive conditions for the stability of the network. A model is first developed using certain approximations, and rate assignment is formulated as a regulation problem in the control framework. The control analysis is then performed to tackle a number of problems concerning the network. The contributions of this work are as follows:

- Using describing function approach to identify limit cycles and hence possible oscillation in the network output.

- Designing an adaptive rate assignment scheme for the case of zero delay in the control loop and a fixed number of active users in the network.

- Obtaining sufficient conditions for network stability under the proposed adaptive rate assignment scheme in the presence of delay.

- Formulating activation and deactivation of the users as a switched system in the context of control, and deriving sufficient conditions for the stability of the network in terms of the speed of activation and deactivation.

- Developing a model for the network with a dynamic rate allocation method.

- Designing a fairness strategy which prioritizes the users according to the subscription fees.

The rest of the thesis is organized as follows. In Chapter 2, some relevant control techniques are studied, which will be used later in the thesis. In Chapter 3, a model is developed for IS-856 uplink, and the control configuration is introduced subsequently. Using a technique similar to the one presented in [29], the oscillation in the network output is analyzed using describing function approach in Chapter 4 , and the effect of allocating two bits to the control signal (instead of just one bit) is discussed in detail. In Chapter 5, an adaptive control scheme is presented for rate 
assignment, and the conditions for the stability of the resulting closed-loop network are derived. The controller is first designed for the case of zero delay and a fixed number of active users in the network. The results obtained are then extended to the most general case. Finally, concluding remarks are drawn in Section 6, followed by some suggested topics for future work. 


\section{Chapter 2}

\section{Background}

\subsection{Introduction}

In this chapter, some important concepts and fundamental techniques from classical control theory are presented, which will be employed later in the thesis. First, a brief description of nonlinear systems is provided and the important notion of limit cycle is introduced. This notion will be used later in the thesis to identify possible oscillations in the network output and to analyze the stability of such oscillations. Then, the stability analysis using Lyapunov theory is studied. This method will be used later in controller design, to derive stability conditions for the closed-loop network. The problem of switched systems will be introduced briefly, followed by some important issues regarding the stability of this type of systems. The concept of switched systems will be used later to model the activation and deactivation of users in a wireless network. Finally, a basic optimization technique which is often used in control systems will be presented. 


\subsection{Nonlinear Systems Analysis}

It is known that all physical systems are nonlinear, in the sense that the relationship between the input and output of the system cannot be characterized by a linear static or dynamic equation. It is often desirable to approximate a nonlinear system with a linear model, because of the powerful analysis and synthesis techniques available for linear systems (due mainly to the superposition principle). Depending on the degree of nonlinearity, however, such an approximation may not be accurate enough in describing the system behavior. Hence, efficient methods are required to study various aspects of nonlinear systems.

There are fundamental differences between linear and nonlinear systems from the control theoretic view. For instance, unlike linear systems, there is no necessary and sufficient condition for the stability of nonlinear systems, in general. More precisely, all stability analysis techniques for general nonlinear systems provide sufficient conditions only. Furthermore, the stability of a nonlinear system may also depend on the initial condition and the equilibrium state, not just the nonlinear dynamics itself. Moreover, for certain types of nonlinearities (which also exist in the wireless networks considered in this thesis), the response of the system can demonstrate permanent oscillations. In order to investigate the behavior of a nonlinear system, it is necessary first to express the dynamics of the system analytically as a set of equations that govern the evolution of the state variables. Such a model can be derived either mathematically from the relationship between different variables of the physical system, or experimentally by using a proper system identification technique. Assume for now that the state-space model of the system is given in 
the form of a finite set of first-order nonlinear (and time-varying) differential equations [23], [6]

$$
\left\{\begin{aligned}
\dot{x}_{1} & =f_{1}\left(t, x_{1}, \ldots, x_{n}, u_{1}, \ldots, u_{p}\right) \\
\dot{x}_{2} & =f_{2}\left(t, x_{1}, \ldots, x_{n}, u_{1}, \ldots, u_{p}\right) \\
& \vdots \\
\dot{x}_{n} & =f_{n}\left(t, x_{1}, \ldots, x_{n}, u_{1}, \ldots, u_{p}\right)
\end{aligned}\right.
$$

where $x_{i}$ and $u_{j}$ are the $i$-th state variable and $j$-th control input, respectively $(i=1, \ldots, n$, and $j=1, \ldots, p)$. Furthermore, $f_{i}$ is a given nonlinear function which characterizes the system dynamics analytically. Define the following vectors

$$
x=\left[\begin{array}{c}
x_{1} \\
x_{2} \\
\vdots \\
x_{n}
\end{array}\right], u=\left[\begin{array}{c}
u_{1} \\
u_{2} \\
\vdots \\
u_{p}
\end{array}\right], f(t, x, u)=\left[\begin{array}{c}
f_{1}(t, x, u) \\
f_{2}(t, x, u) \\
\vdots \\
f_{n}(t, x, u)
\end{array}\right]
$$

(note that $x_{i}$ and $u_{i}$ are functions of time, but the time argument is omitted in the above equations for brevity). Using the above vectors, the system equations given by $(2.1)$ can be rewritten in the following form

$$
\dot{x}=f(t, x, u)
$$

For simplicity and without loss of generality, assume that the system is timeinvariant (or autonomous [6], [23], and that $x$ is the only independent variable in the system function $f(\cdot)$ (e.g., $u$ is either a function of $x$, or a prescribed function of time). In this case, the state equation can be expressed as

$$
\dot{x}=f(x)
$$

Definition 1. [23] The state $x^{*}$ is called an equilibrium point of the system, if $x(t)=x^{*}, \forall t>t_{0}$, provided $x\left(t_{0}\right)=x^{*}$. It is to be noted that a nonlinear system can have multiple equilibrium points, in general; in other words, $x^{*}$ is not necessarily 
unique. For the autonomous system given by (2.4), the equilibrium points are the real roots of the following equation

$$
f(x)=0
$$

The notion of a limit cycle is introduced to characterize the possible oscillation in a nonlinear system.

Definition 2. [23] An oscillation of fixed amplitude and frequency in a nonlinear system is called a limit cycle. Limit cycle is a unique feature of a nonlinear system, and is classified as stable, unstable and semi-stable.

i) A stable limit cycle: All trajectories in the vicinity of the limit cycle will converge to it.

ii) An unstable limit cycle: All trajectories in the vicinity of the limit cycle will diverge from it.

iii) A semi-stable limit cycle: Some of the trajectories in the vicinity of it converge to it, while others diverge from it.

\subsection{Lyapunov Theory}

The first and the most important question about a control system is whether it is stable, since unstable systems are useless and potentially dangerous. One of the most popular and effective approached for studying the stability of a nonlinear system is the Lyapunov theory. In this method the stability of a nonlinear system is checked by constructing a scalar function for the system and monitoring the function's time variation. Some important definitions which will be used later in the thesis are presented in the sequel. 
Definition 3. The equilibrium state $x=0$ is called stable, if for any $R>0$ there exists $r>0$ such that if $\|x(0)\|<r$, then $\|x(t)\|<R$ for all $t \geq 0$, where $\|$. denotes the norm operator. Otherwise, the equilibrium point is unstable.

Definition 4. The equilibrium point $x=0$ is asymptotically stable if it is stable, and there exists $r>0$ such that $\|x(0)\|<r$ implies that $x(t) \rightarrow 0$ as $t \rightarrow \infty$. The ball $\|x(0)\|<r$ is often called the domain of attraction for a specific equilibrium point. In other words, the domain of attraction represents the set of all points such that if selected as the initial point, the trajectories will finally converge to the origin. If the domain of attraction is the entire state space, then the system is referred to as globally asymptotically stable; otherwise, it is called locally asymptotically stable [6].

Definition 5. The equilibrium point $x=0$ is exponentially stable if there exist two strictly positive numbers $\alpha$ and $\lambda$ such that

$$
\|x(t)\| \leq \alpha\|x(0)\| e^{-\lambda t}, \forall t>0
$$

where the number $\lambda$ is called the rate of exponential convergence [6].

Definition 6. A function $f(x, t)$, where $f: D \times[a, b] \rightarrow \mathbb{R}^{n}$ for a region $D \subseteq \mathbb{R}^{n}$, is said to be continuously differentiable over $D \times[a, b]$ if both $f(x ; t)$ and $\frac{\partial f}{\partial x}$ are continuous over $D \times[a, b]$.

Definition 7. A continuously differentiable function $V: \mathbb{R}^{n} \rightarrow \mathbb{R}$ is said to be positive definite in a region $D$ of $\mathbb{R}^{n}$ that contains the origin if

- $V(x)>0, \forall x \in D, x \neq 0$

- $V(x)=0$ if and only if $x=0$, and

- all subsets of $V$ are bounded.

Moreover, $V$ is positive semi-definite if $V(x) \geq 0, \forall x \in D, x \neq 0 . \quad V$ is negative definite, if the first condition is replaced by $V(x)<0$ and negative semi-definite if 
it is replaced by $V(x) \leq 0$. Similarly, a symmetric matrix $A_{n \times n}$ is positive definite if for any non-zero vector $x \in C^{n}$

$$
\operatorname{Re}\left\{x^{*} A x\right\}>0
$$

where $x^{*}$ is the conjugate transpose of the vector $x$. For a real symmetric matrix $A$, the condition (2.7) can be simplified as follows

$$
x^{T} A x>0
$$

It can be shown that all eigenvalues of a positive definite matrix $A$ are in the open right-half plane (RHP).

Definition 8. A real-valued function $f$ defined on a subset of real numbers $D \subseteq \mathbb{R}$, $f: D \rightarrow \mathbb{R}$ is called Lipschitz continuous if a constant $B>0$ can be found such that for any $x_{1}, x_{2}$ in $D$ the following inequality is valid

$$
\left|f\left(x_{1}\right)-f\left(x_{2}\right)\right| \leq B|| x_{1}-x_{2}||
$$

The function $f$ is called locally Lipschitz continuous if for any $x$ in $D$ a neighborhood of $x$ can be found such that $f$ restricted to this neighborhood is Lipschitz continuous.

Consider the system

$$
\dot{x}=f(x), x \in \mathbb{R}
$$

where $f: D \rightarrow \mathbb{R}^{n}$ is locally Lipschitz, and $D \subseteq \mathbb{R}^{n}$ is a domain that contains the origin. Moreover, it is assumed that the origin is the equilibrium point of the system (2.10). Let $V: D \rightarrow \mathbb{R}$ be a continuously differentiable function, positive definite in $D$. The Lyapunov theory states that if $\dot{V}(x)=\frac{\partial V}{\partial x}$ is negative semi-definite, then $x=0$ is a stable equilibrium point and if $\dot{V}(x)=\frac{\partial V}{\partial x}$ is negative definite, then $x=0$ is an asymptotically stable equilibrium point. Here $V$ is called the Lyapunov function [23], [6]. Note that the Lyapunov theory provides sufficient conditions only for stability or asymptotic stability of an equilibrium point. 


\subsection{Switched Systems}

Switched systems are continuous-time systems with discrete switching events. In other words, switched systems consist of multiple modes of operation, orchestrated by a switching rule that determines the active mode of operation (subsystem) at each instant of time. Switching events in switched systems can be classified as state-dependent and time-dependent, or autonomous (uncontrolled) and controlled switching. In the formulation of wireless networks, time-dependent switching will be used in this thesis [15].

The stability of a switched system has been extensively investigated in the literature [24]. As a simple approach, one can seek a common Lyapunov function whose derivative along the states of all systems satisfies the desired inequalities. Considering $\left\{t_{1}, t_{2}, t_{3}, \cdots\right\}$ as a sequence of strictly increasing impulse time, the general impulsive system can be modeled as follows

$$
\begin{cases}\dot{x}(t)=f(x(t)), & t \neq t_{k}, \forall k \in \mathbb{N} \\ x(t)=g\left(x\left(t^{-}\right)\right), & t=t_{k}, \quad \forall k \in \mathbb{N}\end{cases}
$$

where $f$ is locally Lipschitz and the function $g$ represents the jump in the states $x(t)$ as a function of $x\left(t^{-}\right)$[16]. It is well known that the switched system is stable if all individual subsystems are stable and the switching is sufficiently slow such that it allows the transient effects to dissipate after each switching. The simplest way to specify slow switching is to restrict the switching signals in a way that the switching times $t_{1}, t_{2}, \cdots$ satisfy the inequality $t_{i+1}-t_{i} \geq \tau, i=1,2,3, \ldots$. The interval $\tau$ is referred to as the dwell time. It can be shown that if all subsystems are stable and the dwell time is sufficiently large, then the switched system is stable. The dwell time can be explicitly calculated from the exponential decay bounds on the transition matrices of each individual subsystems. Note that one can also achieve stability by allowing fast but limited switchings then letting the Lyapunov function 
of the last subsystem to decay until it reaches to a desired region [15].

\subsection{Describing Function Technique}

It is known that frequency domain analysis is very useful for LTI systems. In particular, the Nyquist method is very effective in determining the stability of an LTI system. Describing function analysis uses the Nyquist stability criterion by considering the nonlinear element as a simple gain (which is a function of the magnitude and frequency of the input signal). Using this technique, one can identify any possible oscillations in the system, which are called limit cycles. Limit cycles is of great importance in analysis and synthesis of nonlinear control systems. Limit cycles are often used in the design of electronic oscillators. However, in most control systems limit cycles are undesirable [23], [6].

One of the useful tools in the analysis of nonlinear control systems is the describing function technique. Any closed-loop control system containing an element with hard nonlinearity such as frictions, hysteresis, saturation actuator, dead zone, etc. can be analyzed using the describing function technique [6]. This technique can be effectively used in identifying limit cycles and their types in a nonlinear control system. The idea behind this technique is that the oscillation in a nonlinear control system described above can be approximated by a sinusoidal signal with a particular frequency and amplitude. In fact, the input to the linear element in Fig. 2.1 can be expanded as the sum of infinitely many harmonics. Since typical control loops have a low-pass filtering property, the high frequency components are filtered out, and the output $y(t)$ is composed mostly of the lower harmonics.

In order to present the describing function method, the following assumptions are required.

Assumption 1. There exists only one nonlinear element in the overall system. 


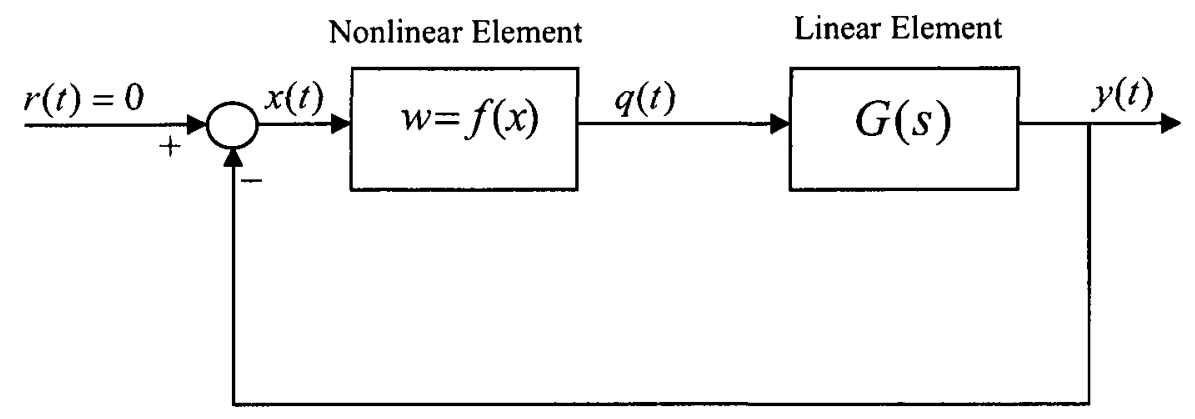

Figure 2.1: A nonlinear control system.

Assumption 2. The nonlinear system is time-invariant.

Assumption 3. Given a sinusoidal input $x(t)=\sin (\omega t)$, only the fundamental component $q_{1}(t)$ is dominant in the output $q(t)$. In other words, the control loop acts as a low-pass filter, attenuating the higher-order harmonics.

Consider a sinusoidal input of amplitude $A$ and frequency $\omega$, which is applied to the nonlinear element in Fig. 2.1. Suppose that the output of the nonlinear element is periodic. Using the Fourier series expansion, the periodic signal $q(t)$ can be written as

$$
q(t)=\frac{a_{0}}{2}+\sum_{n=1}^{\infty}\left(a_{n} \cos (n \omega t)+b_{n} \sin (n \omega t)\right)
$$

The Fourier coefficients $a_{i}$ and $b_{i}$ can be obtained from the following integrals

$$
\begin{aligned}
& a_{0}=\frac{1}{\pi} \int_{-\pi}^{\pi} q(t) d(\omega t) \\
& a_{n}=\frac{1}{\pi} \int_{-\pi}^{\pi} q(t) \cos (n \omega t) d(\omega t) \\
& b_{n}=\frac{1}{\pi} \int_{-\pi}^{\pi} q(t) \sin (n \omega t) d(\omega t)
\end{aligned}
$$

From Assumption 3, only the fundamental component $q_{1}(t)$ is supposed to be dominant, i.e.,

$$
q(t) \simeq q_{1}(t)=a_{1} \cos (\omega t)+b_{1} \sin (\omega t)=M \sin (\omega t+\phi)
$$


where

$$
\begin{aligned}
& M(A, \omega)=\sqrt{a_{1}^{2}+b_{1}^{2}} \\
& \phi(A, \omega)=\arctan \left(\frac{a_{1}}{b_{1}}\right)
\end{aligned}
$$

Now, the describing function of the nonlinear element is defined as the complex ratio of the fundamental component of the nonlinear element output to the input sinusoid. In other words

$$
N(A, \omega)=\frac{M e^{j(\omega t+\phi)}}{A e^{j \omega t}}=\frac{M}{A} e^{j \phi}=\frac{1}{A}\left(b_{1}+j a_{1}\right)
$$

It is to be noted that the describing function is real because $a_{1}$ is zero for odd nonlinearities.

\subsubsection{Prediction of Existence of Limit Cycles}

Assume that there exists an oscillation of amplitude $A$ and frequency $\omega$ in the system of Fig. 2.1. Therefore, the variables in the loop should satisfy the following:

$$
\begin{aligned}
& x=-y \\
& q=N(A, \omega) x \\
& y=G(j \omega) q
\end{aligned}
$$

Therefore, $y=G(j \omega) N(A, \omega)(-y)$, which can be written as

$$
G(j \omega)=-\frac{1}{N(A, \omega)}
$$

As a result, the amplitude $A$ and frequency $\omega$ of the limit cycle in the system must satisfy (2.18). The idea is to plot both sides of (2.18) in the complex plane and find the intersection points of the two curves which correspond to the possible limit cycles. One can also check the stability of the limit cycles using the extended Nyquist criterion [23]. 


\subsection{Quasi-Newton Methods in Optimization}

In order to find local maximum or minimum of a given function quasi-Newton methods are developed in the literature, which use Newton's approach to find the stationary point of a function. In quasi-Newton methods, the Hessian matrix of second derivatives of the function to be minimized does not need to be computed since the full Hessian matrix is usually difficult to calculate in practice. Instead, these methods use an approximation of the Hessian matrix. The well-known quasi-Newton algorithms are the rank-one correction Davidon Fletcher Powell (DFP) and Broyden Fletcher Goldfarb Shanno (BFGS) [25].

Definition 9. The gradient (or gradient vector field) of a scalar function $f\left(x_{1}, x_{2}, x_{3}\right.$, $\left.\cdots, x_{n}\right)$ is denoted by $\nabla f$ or $\vec{\nabla} f$, where $\nabla$ represents the vector differential operator. The gradient of $f$ is defined as a the vector field whose components are the partial derivatives of $f$, i.e.:

$$
\nabla f=\left(\frac{\partial f}{\partial x_{1}}, \cdots, \frac{\partial f}{\partial x_{n}}\right)
$$

Definition 10. The Hessian matrix is a square matrix whose elements are the second partial derivatives of a given function, and it describes the local curvature of a function of many variables. For a real-valued function $f\left(x_{1}, x_{2}, \cdots, x_{n}\right)$, the Hessian matrix can be written as follows:

$$
H(f)=\left[\begin{array}{cccc}
\frac{\partial^{2} f}{\partial x_{1}^{2}} & \frac{\partial^{2} f}{\partial x_{1} \partial x_{2}} & \cdots & \frac{\partial^{2} f}{\partial x_{1} \partial x_{n}} \\
\frac{\partial^{2} f}{\partial x_{2} \partial x_{1}} & \frac{\partial^{2} f}{\partial x_{2}^{2}} & \cdots & \frac{\partial^{2} f}{\partial x_{2} \partial x_{n}} \\
\vdots & \vdots & \vdots & \vdots \\
\frac{\partial^{2} f}{\partial x_{n} \partial x_{1}} & \frac{\partial^{2} f}{\partial x_{n} \partial x_{2}} & \cdots & \frac{\partial^{2} f}{\partial x_{n}^{2}}
\end{array}\right]
$$

Consider the problem of minimizing the function $f(\underline{x})$, and let following iterative algorithm be used:

$$
\underline{x}_{k+1}=\underline{x}_{k}-\alpha_{k} S_{k} \nabla f^{T}\left(\underline{x}_{k}\right)
$$


where $\alpha_{k}$ is chosen to minimize $f\left(\underline{x}_{k}-\alpha_{k} S_{k} \nabla f^{T}\left(\underline{x}_{k}\right)\right)$ and $S_{k}$ is a symmetric $n \times n$ matrix. It is desired at each iteration to choose $S_{k}$ as close as possible to $F^{-1}\left(\underline{x}_{k}\right)$, i.e., the inverse of the Hessian matrix.

\section{Algorithm 1.}

Initialization: Start with any symmetric positive definite matrix $H_{0}$ and vector $\underline{x}_{0}$ and set $k=0$. Then:

i) Calculate

$$
\underline{d}_{k}=-H_{k} \cdot \underline{g}_{k}
$$

where $\underline{g}_{k}$ is the transpose of the gradient of the function to be minimized. Find the parameter $\alpha$ of the following optimization problem using any line search:

$$
\min f\left(\underline{x}_{k}+\alpha \underline{d}_{k}\right), \alpha>0
$$

ii) Set $\underline{x}_{k+1}=\underline{x}_{k}+\alpha \underline{d}_{k}, \underline{p}_{k}=\alpha_{k} \cdot \underline{d}_{k}$, and $\underline{g}_{k+1}=\underline{g}\left(\underline{x}_{k+1}\right)$

iii) Set $\underline{q}_{k}=\underline{g}_{k+1}-\underline{g}_{k}$ and update the approximation of Hessian matrix as follows:

$$
H_{k+1}=H_{k}+\left(1+\frac{\underline{q}_{k}^{T} H_{k} \underline{q} \underline{q}_{k}}{\underline{q}_{k}^{T} \cdot \underline{p}_{k}}\right)\left(\frac{\underline{p}_{k} \cdot \underline{p}_{k}^{T}}{\underline{p}_{k}^{T} \underline{q}_{k}}\right)-\frac{\underline{p}_{k} \cdot \underline{q}_{k}^{T} \cdot H_{k}+H_{k} \underline{q}_{k} \cdot \underline{p}_{k}^{T}}{\underline{q}_{k}^{T} \cdot \underline{p}_{k}}
$$

Replace $k$ with $k+1$ and go to step (i). 


\section{Chapter 3}

\section{Interference Model for IS-856}

\section{Uplink}

\subsection{Introduction}

In this chapter, a model is developed for IS-856 uplink, and the rate assignment in the network is formulated as a regulation problem in the context of feedback control system. In this model, the one-bit control signal transmitted from the base station in represented by an on-off block (which is a nonlinear element), and the mapping from the feasible rate set to the feasible $T 2 P_{i}(t)$ set is expressed in a modified form for practical purposes. Important definitions and assumptions are introduced, and the rate assignment objectives are presented subsequently.

\subsection{Access Terminal}

Access terminals (AT) are the mobile components of the network that provide data to users. Access network (AN), on the other hand, is the fixed element of the network, i.e., a base station which provides data for ATs. A CDMA channel in an AN along with the corresponding covered area is called a sector [7], [10]. 
Let the number of ATs available in a sector of an IS-856 network be denoted by $n$, and assume that all of these ATs are full-buffered. Assume also that the base station receives the same pilot power from all ATs [10], [11], [14]; this strategy is often called perfect power control and is studied in a number of papers such as [21], [34], [35].

The ATs transmit data with a rate $R_{i}(t) \in \Gamma, \forall t>0$, where $\Gamma$ is the set of all feasible rates (which is a known standard set) [19]. Let $T 2 P_{i}(t)$ (traffic to pilot ratio) denote the ratio of the total data power to the pilot power for the $i$-th AT; this ratio belongs to a given feasible set $\Lambda$. Define a one-to-one mapping $F(\cdot)$ from the feasible rate set $\Gamma$ to the feasible $T 2 P$ set $\Lambda$ as shown in Fig. 3.1, and denote it as [7]

$$
T 2 P_{i}(t)=F\left(R_{i}(t)\right)
$$

The total traffic power of the $i$-th $\mathrm{AT}$ is then given by

$$
P_{i}(t)=T 2 P_{i}(t) \pi_{i}(t)
$$

where $\pi_{i}(t)$ denotes the pilot power of the $i$-th AT.

\subsection{Access Network}

Let $N_{0} W$ represent the total background noise, which is assumed to be Gaussian. The raise over thermal (ROT) in the base station at any time instant $t$ is defined as $[10],[20]$

$$
Z(t)=10 \log _{10}\left(\frac{I(t)+J(t)+N_{0} W}{N_{0} W}\right)
$$

where $J(t)$ is the out-sector interference coming from the ATs that are not in soft hand-off with the base station. Furthermore, $I(t)$ is the total in-sector interference which is given by

$$
I(t)=\sum_{i=1}^{n} T 2 P_{i}(t) P_{\text {pilot }}
$$




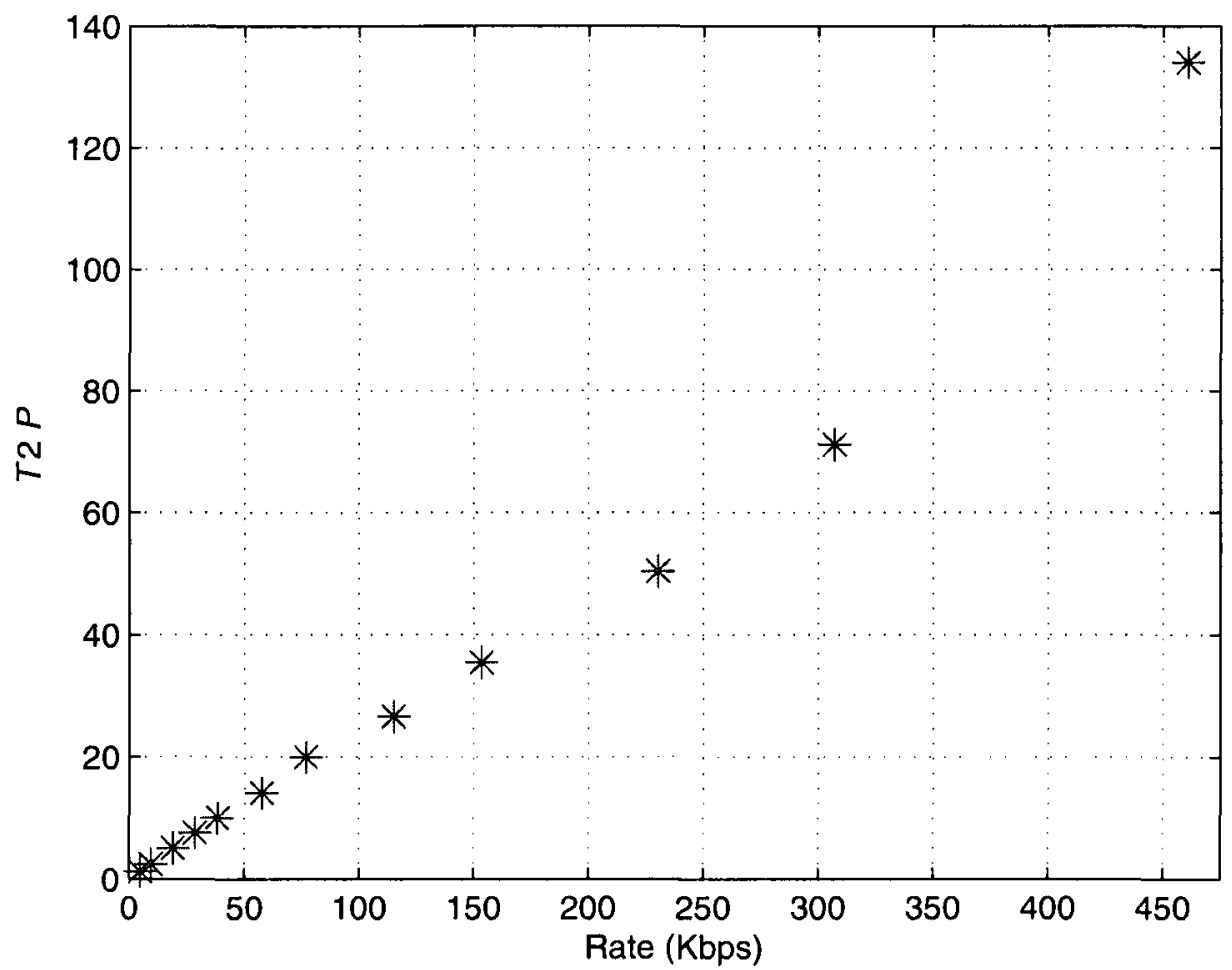

Figure 3.1: The mapping $F(\cdot)$ from the feasible rate set $\Gamma$ to the feasible $T 2 P$ set $\Lambda$. 
where $P_{\text {pilot }}$ is the received pilot power from each AT at the AN, in the case of perfect power control.

On one hand, the existence of strong interference in the network results in high ROT, which has performance implications. On the other hand, weak interference in the network leads to low ROT, which can be interpreted as under-utilization of the network resources. Therefore, it is desired to keep the ROT close to a prescribed threshold denoted by $Z_{t h}[33]$.

By substituting $T 2 P_{i}(t)$ in (3.4) with (3.1) and assuming that the out-sector interference $J(t)$ in (3.3) is negligible compared to $I(t)$ for all $t$, one can conclude that the term $\sum_{i=1}^{n} F\left(R_{i}(t)\right)$ (which is the feedback signal from the ATs to the AN) should be regulated to a reference value $Z_{r}$ given below [11], [40]

$$
Z_{r}=\frac{N_{0} W}{P_{\text {pilot }}}\left(10^{Z_{t h} / 10}-1\right)
$$

The feedback signal received by in the AN is compared to the reference $Z_{r}$ provided above, and the error signal

$$
e(t)=Z_{r}-\sum_{i=1}^{n} F\left(R_{i}(t)\right)
$$

is produced. This error signal then passes through a two-level comparator, generating a signal called reverse activity bit (RAB) [7]; this process is formulated below

$$
U(t)=\operatorname{sgn}(e(t))
$$

This means that if the overall rate of the ATs is less than a desired value, then $U(t)=1$; otherwise, $U(t)=-1$. The objective is to design a set of controllers to regulate the rate of the ATs such that $I(t)$ reaches the desired level in a reasonably short period of time. The closed-loop network configuration is shown in Fig. 3.2 as a feedback control system.

Remark 1. Throughout this thesis, the signal $U(t)$ is referred to as control signal. This notion should not be confused with the output of the controllers in Fig. 3.2, as this term is often used to describe the controller output in the control literature. 


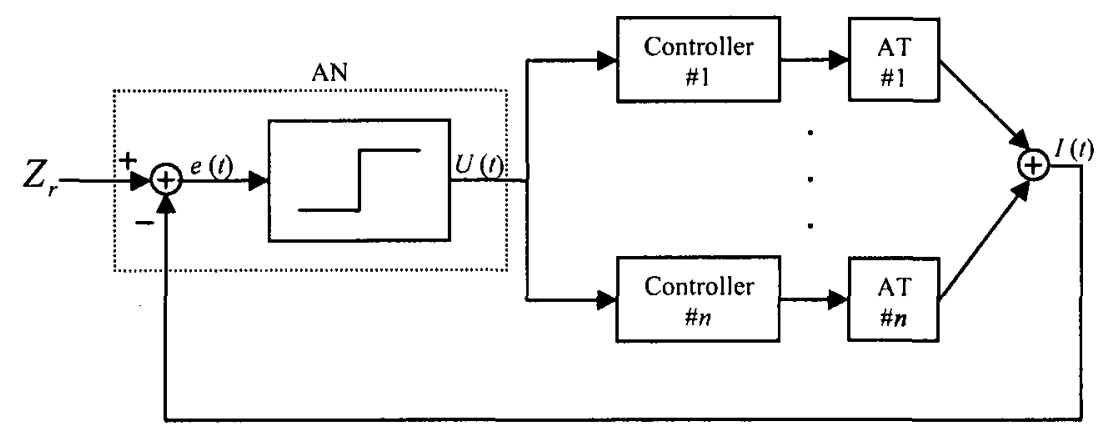

Figure 3.2: Rate-control loop in IS-856 uplink, with $n$ active users available in the sector.

Remark 2. The assumption of $J(t)$ being negligible compared to $I(t)$ in (3.3) is only made to simplify the analysis (i.e. as in the case of a single sector network) [10], [11], [13], [14]. However, in some practical settings this may not be a realistic assumption.

Remark 3. Note that the output of the controller can take any real value, but according to the mapping $F(\cdot)$ shown in Fig. 3.1, the domain of this mapping (i.e., the allowable values of its input) is limited to the finite set $\Gamma$. In order to overcome this difficulty, a new mapping $\tilde{F}(\cdot)$ is introduced whose domain is all positive real values between 0 and 500 Kbps. This mapping is obtained by considering a piecewise constant T2P, where each discontinuity is centered at a point between two feasible rates in the set $\Gamma$. This leads to a mapping $\tilde{F}(\cdot)$ shown in Fig. 3, which is, in fact, a nonuniform quantizer. 


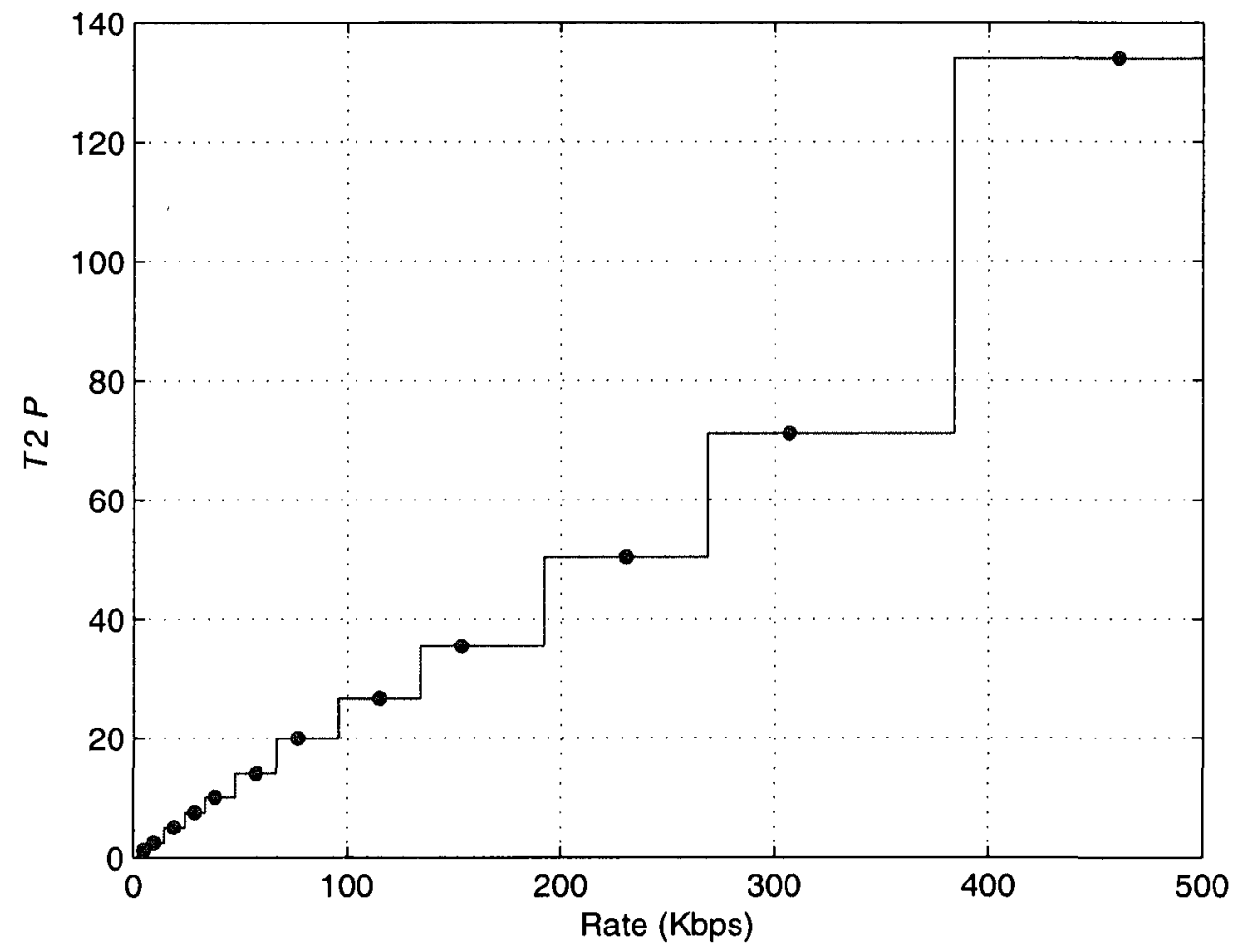

Figure 3.3: The nonuniform quantizer $\tilde{F}(\cdot)$ as the pragmatic counterpart of the mapping $F(\cdot)$ (solid circles indicate the corresponding feasible set in the mapping $F)$. 


\section{Chapter 4}

\section{Limit Cycle Analysis in Rate Assignment Control Loop}

\subsection{Introduction}

In this chapter, the describing function technique is introduced for the rate assignment in IS-856, using the results in [14]. The possible oscillation of the system due to the existence of a nonlinear element in the AN is identified in the framework of limit cycle analysis. The stability of the oscillation is verified using Nyquist criterion, and the effect of controller parameters on the magnitude of oscillation and the transient performance are discussed. Furthermore, it is shown that using a three-level quantization for the comparison error instead of a simple on-off element (corresponding to a single-bit control signal transmitted from the base station to the ATs) would significantly improve the rate assignment performance. 


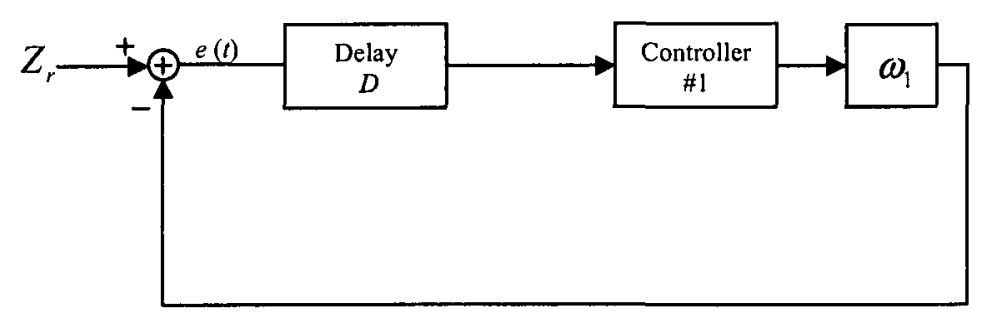

Figure 4.1: The uplink rate control loop of IS-856.

\subsection{Oscillation in Network Output: Limit Cycle Analysis}

As the first step in limit cycle analysis, assume that there is no limitation in broadcasting the control signal from the base station (AN) to the ATs, i.e., the nonlinear relay in AT in Fig. 3.2 is removed. Thus,

$$
A N(s)=1
$$

Assume also that the mapping $F(\cdot)$ is approximated by a first-order curve, and hence it can be replaced by a simple gain $\omega_{1}$. In other words, the mapping $F(\cdot)$ is to be approximated by the closest line of the form

$$
\tilde{F}_{1}=\omega_{1} x_{i 2}(t)+\omega_{0}
$$

With these assumptions, the system to be controlled is, in fact, modeled by a simple delay $z^{-D}$ followed by a constant gain $\omega_{1}$, as shown in Fig. 4.1 for one AT.

One can design a discrete-time controller $G(z)$ to place the poles of the resultant closed-loop system in the desired locations in the complex plane. It follows from the internal model principle [41], [42] that in order to achieve zero steady-state error for a step input, an accumulator (the discrete-time equivalent of an integrator) is required in the forward path. Similarly, if the input $Z_{r}$ has a ramp component then it is required that $G(z)$ to have two poles at $z=1$. However, one should 
ensure that adding integrator(s) to the forward path would not lead to closed-loop instability.

Given the single AT with the structure shown in Fig. 4.1, it is desired to design an LTI controller using classical control methods, for the case when the delay includes two frames. Let a controller of the following form which consists of an accumulator (for steady-state performance) and a lead compensator (for transient performance) be used:

$$
G(z)=K \frac{1-z_{0} z^{-1}}{\left(1-z^{-1}\right)\left(1-p_{0} z^{-1}\right)}
$$

For a $5 \%$ overshoot, the closed-loop poles can be placed at the following locations: $z_{1}=0.248+j 0.752, z_{2}=0.248-j 0.752, z_{3}=-0.196$. This can be achieved by choosing $p_{0}=-0.7, z_{0}=-0.1$, and $K=1.23$. To check stability, the root locus trajectories of the closed-loop system is depicted in Fig. 4.2. It can be verified that the system with the controller (4.3) is stable for $0<K<1.625$.

Consider now the on-off block in the AN in Fig. 3.2 (which reflects minimal communication between ATs and AN), where the base station broadcasts the sign of the comparison error (3.7), instead of the error signal itself. In this case, the control block of AN is, in fact, a relay, and has applications in many practical systems.

In order to study the stability of this control strategy, one can use describing function analysis to investigate the existence of possible limit cycles in the closedloop system and their characteristics (in terms of stability). Assume that if the error signal right before the relay block is the periodic signal $e(t)=A \sin (\omega t)$, then the output of the relay will also be a periodic signal that can be expressed using the Fourier series expansion as follows

$$
U(t)=n(\omega t)=\frac{A_{0}}{2}+\sum_{k=1}^{\infty} A_{k} \cos (k \omega t)+\sum_{k=1}^{\infty} B_{k} \sin (k \omega t)
$$

Since $U(t)$ is an odd function (as the sign function of the odd function $e(t)$ ), (4.4) 


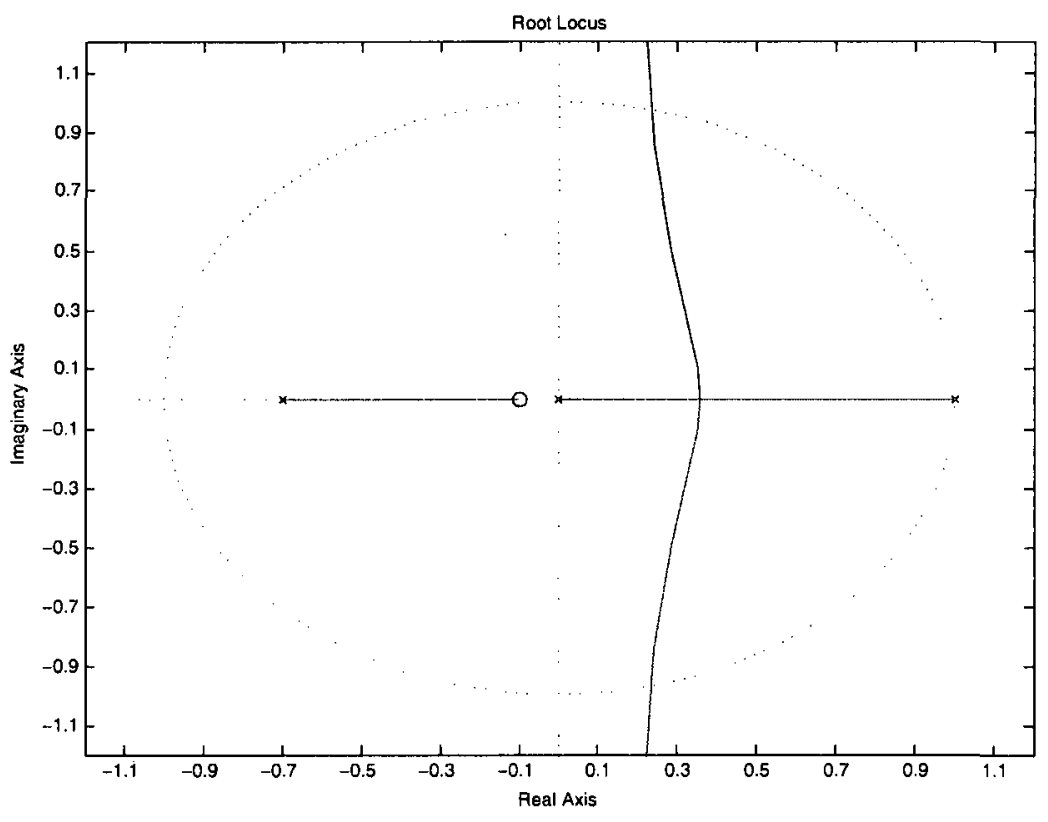

Figure 4.2: The root locus trajectories of the IS-856 uplink controller using the discrete-time LTI control law (4.3).

can be simplified as

$$
n(\omega t)=\sum_{k=1}^{\infty} B_{k} \sin (k \omega t)
$$

Assume that the loop transfer function acts as a low-pass filter that eliminates the high-frequency harmonics of (4.4), and hence only the main harmonic of (4.4) passes through the loop (this is a realistic assumption in typical control systems). Therefore, the describing function of the relay element can be written as

$$
N(j \omega, A)=\frac{B_{1}}{A} \angle 0
$$

where $B_{1}$ can be calculated as

$$
B_{1}=\frac{1}{\pi}\left(\int_{-\pi}^{0}-\sin (\alpha) d \alpha+\int_{0}^{\pi} \sin (\alpha) d \alpha\right)
$$

Thus, the describing function of the relay can be simplified to

$$
N(A)=\frac{4}{\pi A}
$$




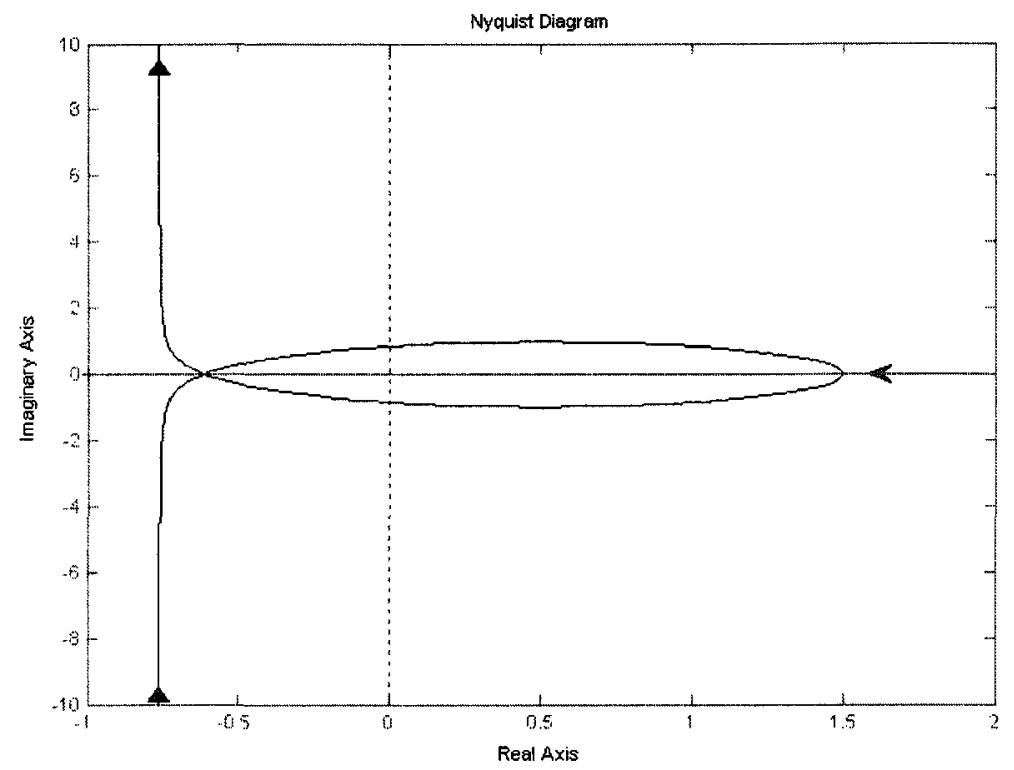

Figure 4.3: The Nyquist diagram of the uplink rate controller along with the describing function of the nonlinear element of AN.

The magnitude and frequency of the limit cycle satisfy the following condition

$$
e^{-j \omega_{0} D_{1}} G\left(j \omega_{0}\right)=-\frac{1}{N(A)}
$$

Now, let the controller (4.3) with the same parameters given earlier be used here. The intersection of the Nyquist diagram of $e^{-j \omega_{0} D} G\left(j \omega_{0}\right)$ and $-\frac{1}{N(A)}$ determines the amplitude and frequency of the limit cycle which is depicted in Fig. 4.3 with blue and red curves, respectively. The Nyquist diagram hits the real axis at -0.61 and 1.5 which correspond to $\omega_{01}=1.34 \mathrm{rad} / \mathrm{sec}$ and $\omega_{02}=\pi \mathrm{rad} / \mathrm{sec}$, respectively.

It is desired now to check the stability of each limit cycle. To this end, consider first the intersection at -0.61 , which corresponds to a limit cycle with amplitude $A_{1}$ and frequency $\omega_{01}$. Let the amplitude of the input to the nonlinear element be slightly disturbed. On one hand, if the amplitude is increased, then the corresponding point on the real axis is no longer encircled by the Nyquist plot of $G(j \omega)$. According to the extended Nyquist criterion [23], since the system is stable in this 
case, the amplitude decreases and as a result the corresponding point on the real axis returns to the original position. On the other hand, if the amplitude of the oscillation decreases, then the corresponding point on the real axis is encircled by the Nyquist of $G(j \omega)$, and hence the system becomes unstable. As a result, the amplitude of the oscillation increases and the point returns to the original position. Therefore, the left intersection corresponds to a stable limit cycle of the frequency $\omega_{0}=1.34$. Using a similar argument, it can be shown that the limit cycle corresponding to the right intersection with the real axis is unstable. It is to be noted that since the trajectory of $-\frac{1}{N(A)}$ covers the whole real axis, a limit cycle is inevitable with this type of controller.

On the other hand, the amplitude of the limit cycle can be obtained from equation (4.9) as follows

$$
e^{-j 2 \times 1.34} G(j 1.34)=-0.616 K=-\frac{\pi A}{4} \Rightarrow A=0.784 K
$$

This means that a greater $K$ leads to a limit cycle with greater amplitude.

To improve the overall control performance, it is desired now to increase the number of bits assigned for the communication between the base station and ATs (i.e., $U(t))$ to two. In the control formulation, this implies that the on-off relay in the $\mathrm{AN}$ is replaced by a four-level nonlinear quantizer. For the simplicity of analysis, however, a three-level quantizer will be used here, which will show that adding one quantization level to the error comparison would significantly improve the control performance.

Using a similar approach, the stability of the limit cycles in the case of a three-level error comparison can be verified using the describing function analysis. Suppose the error signal before the nonlinear element is purely sinusoidal as depicted 


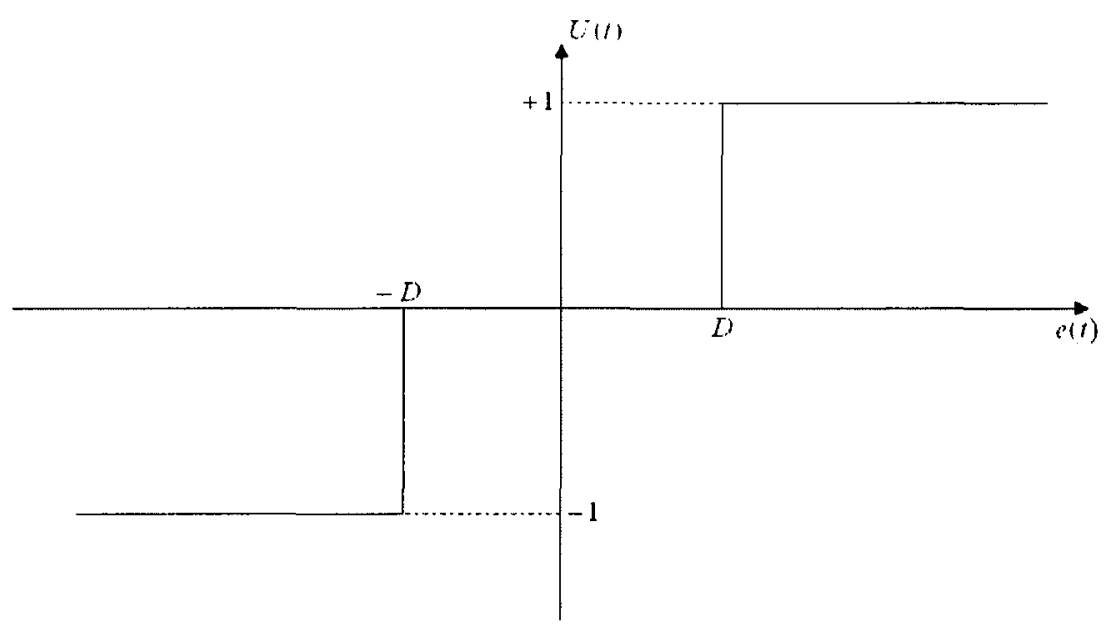

Figure 4.4: A three-level nonlinear A/D block at the AT.

in Fig. 4.5. Therefore, the output signal $U(t)$ can be written as

$$
U(t)= \begin{cases}0, & 0 \leq \omega t \leq m \\ 1, & m \leq \omega t \leq \pi-m \\ 0, & \pi-m \leq \omega t \leq \pi+m \\ -1, & \pi+m \leq \omega t \leq 2 \pi-m \\ 0, & 2 \pi-m \leq \omega t \leq 2 \pi\end{cases}
$$

where $m$ is the angle (in radians) at which the amplitude of the sinusoidal error signal $e(t)$ reaches the level $D$ (i.e., $m=\arcsin \left(\frac{D}{A}\right)$ ). This signal can be expressed using Fourier series as follows:

$$
U(t)=n(\omega t)=\frac{A_{0}}{2}+\sum_{k=1}^{\infty} A_{k} \cos (k \omega t)+\sum_{k=1}^{\infty} B_{k} \sin (k \omega t)
$$

Since $U(t)$ is an odd signal, thus $A_{i}=0, i=0,1,2, \ldots$ Therefore, (4.12) can be rewritten as

$$
n(\omega t)=\sum_{k=1}^{\infty} B_{k} \sin (k \omega t)
$$




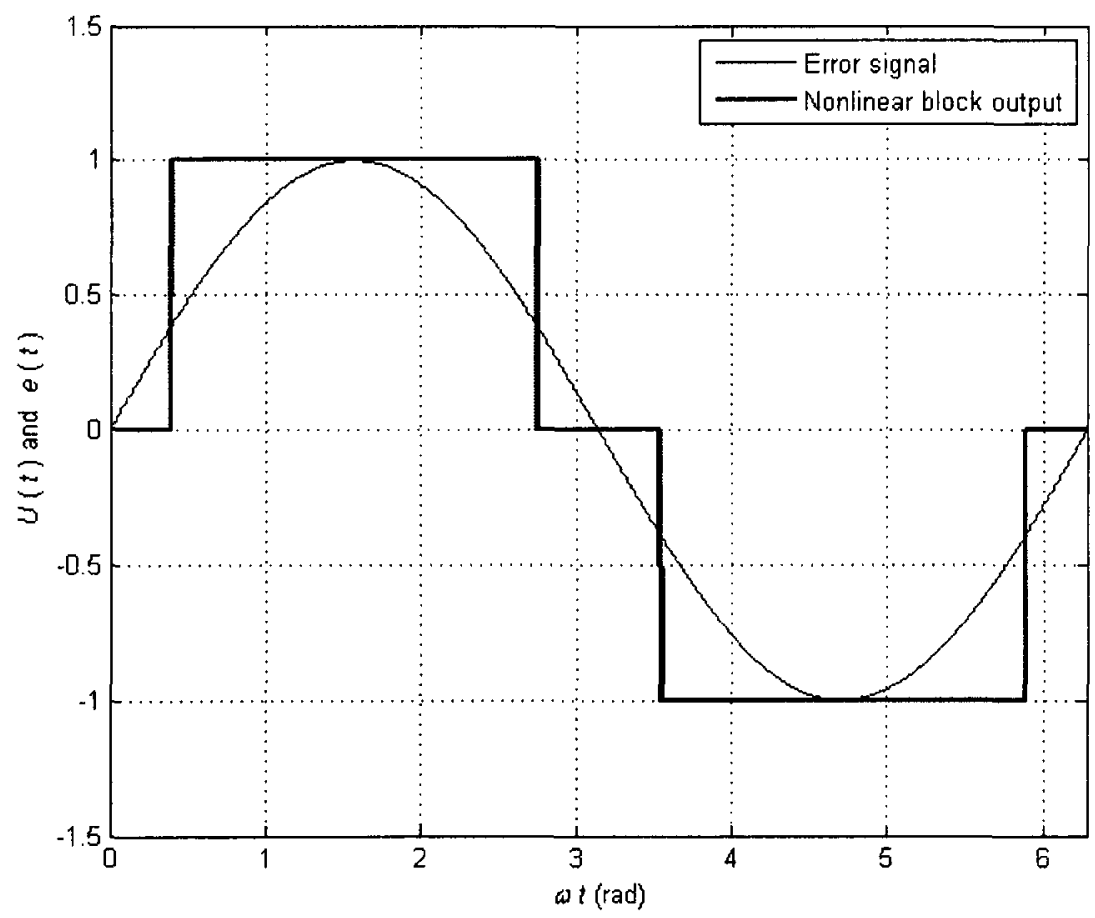

Figure 4.5: Typical input and output signal of a three-level quantizer in the AN. 
Following a discussion similar to that provided in the previous case, the describing function of the three-level quantizer is obtained as

$$
N(j \omega, A)=\frac{B_{1}}{A} \angle 0
$$

The symmetric property of $U(t)$ over the four quarters of a period yields

$$
B_{1}=\frac{4}{\pi} \int_{0}^{\frac{\pi}{2}} U(t) \sin (\omega t) d t=\frac{4}{\pi}\left(\int_{m}^{\frac{\pi}{2}} \sin (\omega t) d t\right)
$$

which can be simplified as

$$
\left.B_{1}=\frac{4}{\pi} \sqrt{(1}-\frac{D^{2}}{A^{2}}\right)
$$

Therefore, the describing function of the three-level quantizer can be written as

$$
N(A)= \begin{cases}0, & A<D \\ \frac{4}{\pi A} \sqrt{\left(1-\frac{D^{2}}{A^{2}}\right)}, & A \geq D\end{cases}
$$

which is depicted in Fig. 4.6 for $D=0.1$.

Again, let the amplitude and frequency of the limit cycle be denoted by $A$ and $\omega$, respectively. As in the case of a two-level quantizer, the parameters $A$ and $\omega$ are related by the following equation

$$
G(j \omega)=-\frac{1}{N(A, \omega)}
$$

Consider the compensator (4.3) with the parameters $z_{0}=-0.1, p_{0}=-0.7$ and $k=0.1$. The corresponding Nyquist diagram as well as the function $-\frac{1}{N(A, \omega)}$ are depicted in Fig. 4.7 in blue and red, respectively.

As it can be seen from Fig. 4.7, the two curves have no intersection. It is to be noted that the Nyquist diagram of $G(j \omega)$ is scaled by $K$; as a result, for a sufficiently large $K$, there is the possibility of having a stable limit cycle. In particular, the Nyquist diagram of $G(j \omega)$ and the real axis intersect at $-0.616 K$. Moreover, the function $-\frac{1}{N(A)}$ covers the portion of real axis from $\infty$ to -0.3 . This 


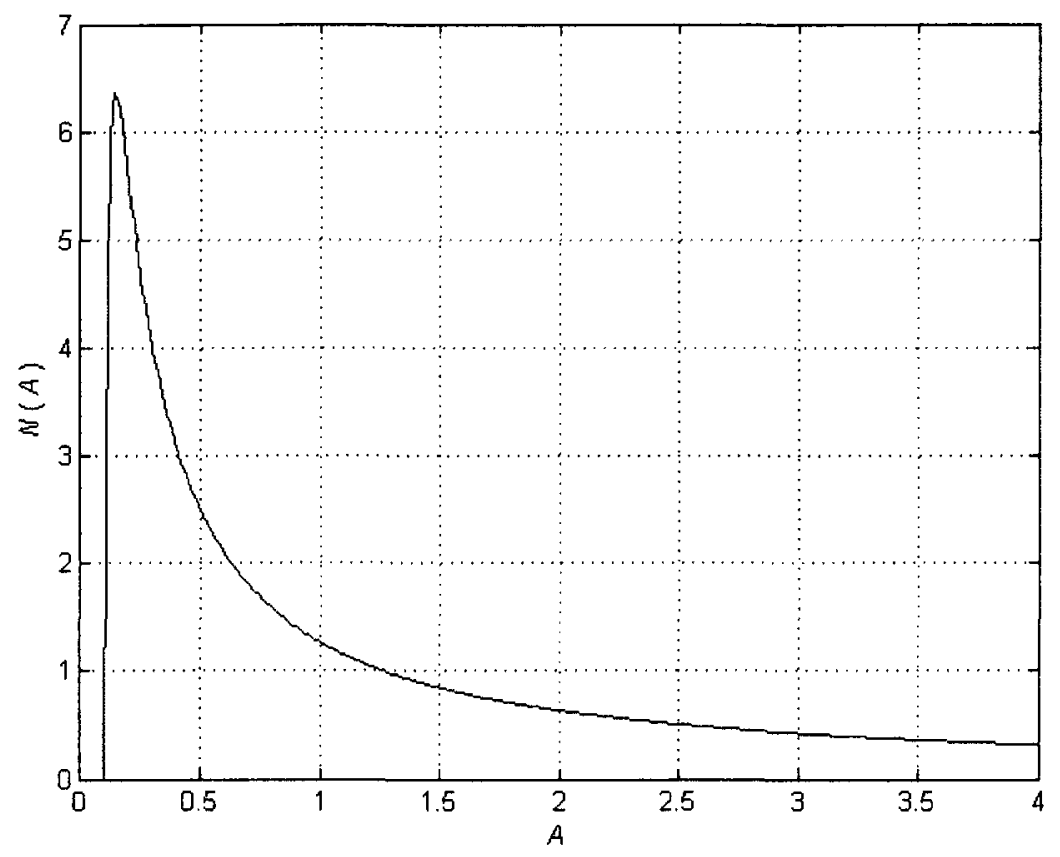

Figure 4.6: The describing function of the three-level quantizer with $D=0.1$.

implies that for any controller gain $K$ smaller than $K_{\text {critical }}$, there will be no limit cycle, where

$$
K_{\text {critical }}=\frac{0.3}{0.616}=0.4870
$$

\subsection{Simulation Results}

Consider first the simple case of no nonlinear element in the network. In this case, the step response of the uplink rate control system is depicted in Fig. 4.8 using the LTI controller (4.3) with the parameters $p_{0}=-0.7, z_{0}=-0.1$, and $K=1.23$.

Now, let the two-level quantizer be used in the AN, along with the controller (4.3) with the parameters $p_{0}=-0.7, z_{0}=-0.1$. The step response of the network is depicted in Figs. 4.9 and 4.10 for two different values of the controller gain $K=0.1$ and $K=0.01$, respectively. It can be seen from these figures that the amplitude of the limit cycle increases by increasing the control gain $K$, as expected from (4.10). 


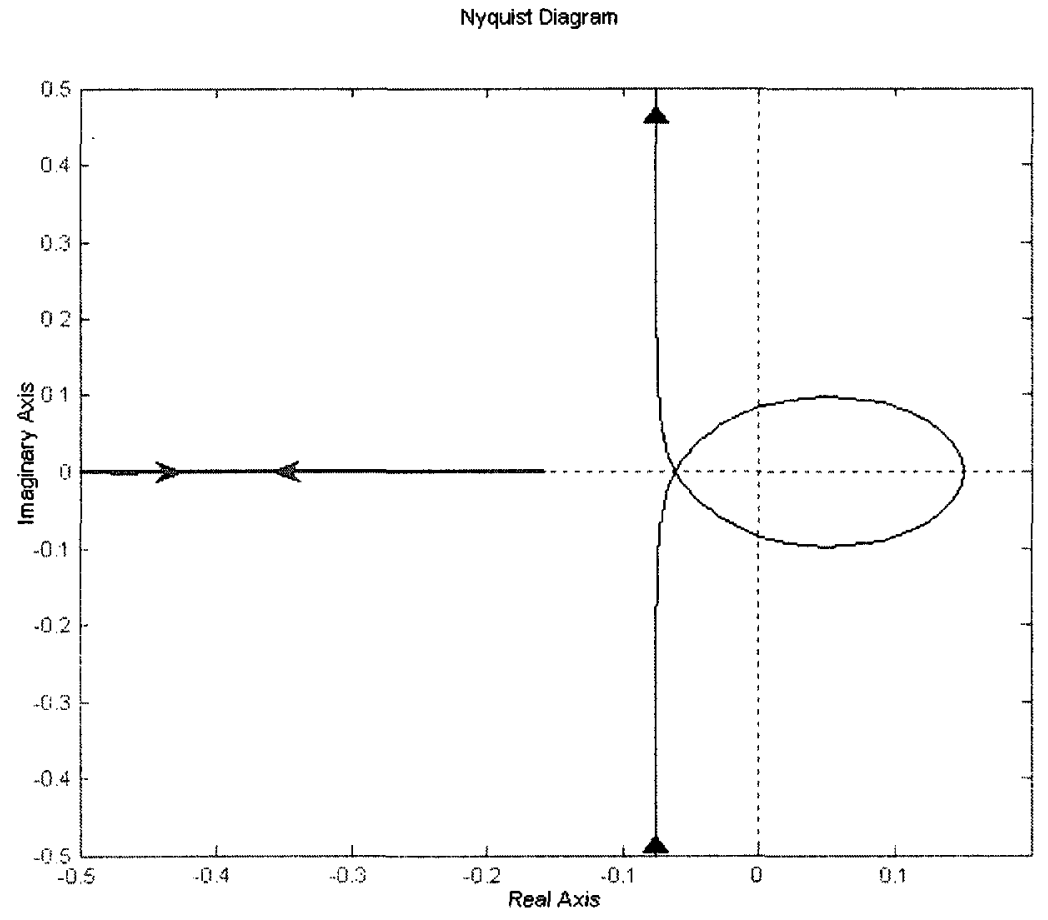

Figure 4.7: The Nyquist diagram of $G(j \omega)$ and $-\frac{1}{N(A, \omega)}$ drawn to identify possible limit cycles in the IS-856 uplink with the control law (4.3) and a three-level error comparison. 


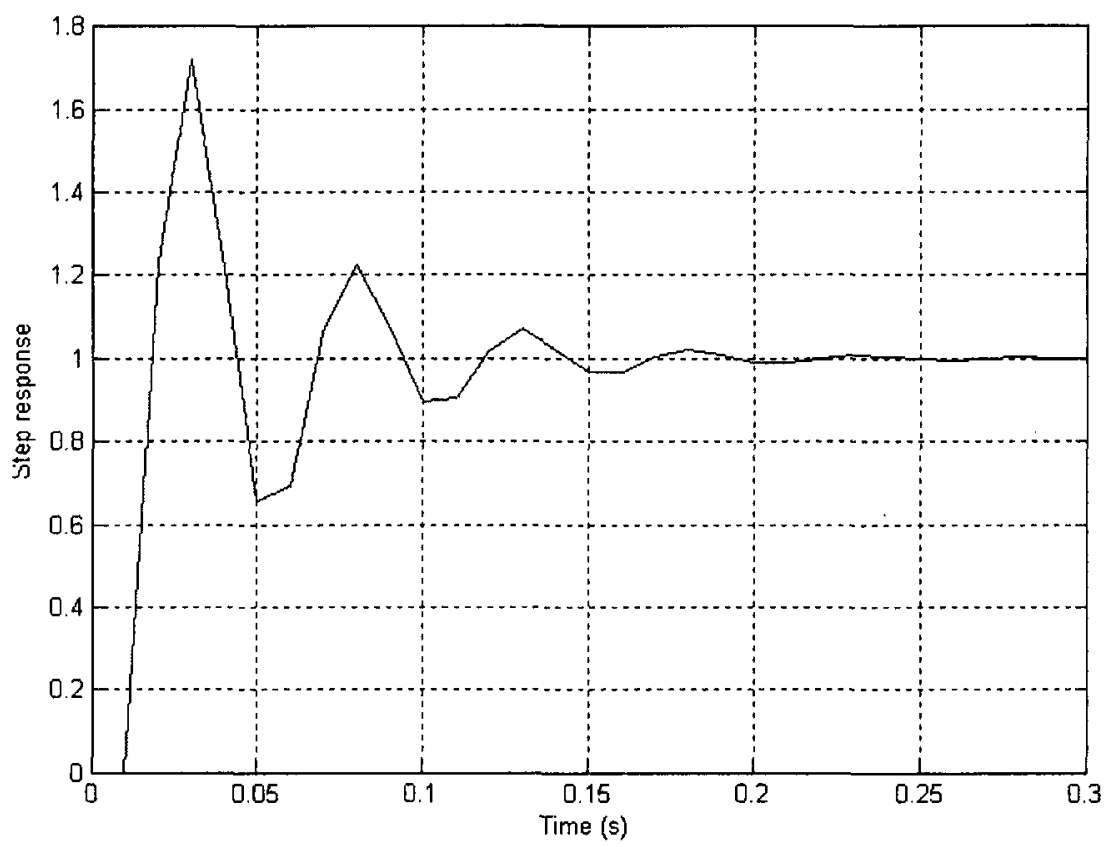

Figure 4.8: The step response of the IS-856 uplink using the control law (4.3) with $K=1.23$ and no nonlinear element in the model.

However, the transient response of the system is faster for a larger value of $K$, which is desirable. This introduces a trade-off in choosing the controller gain $K$.

One can also use a proper optimization technique to find a controller which minimizes a prescribed performance index. It is first required to choose a cost function which reflects the design objectives. Then, an appropriate numerical or analytical technique needs to be adopted to minimize the cost function. For instance, consider the following cost function:

$$
J=\int_{0}^{T} t e^{2}(t) d t
$$

This cost function reflects the energy of error, with a weighting which increases by time. In other words, the objective is to reduce the error as quickly as possible. One can use the Broyden Fletcher Goldfarb Shanno (BFGS) optimization method to minimize $J$ [25]. This method is known to be superior to other existing techniques in terms of accuracy and the convergence rate. Like any other numerical method, it 


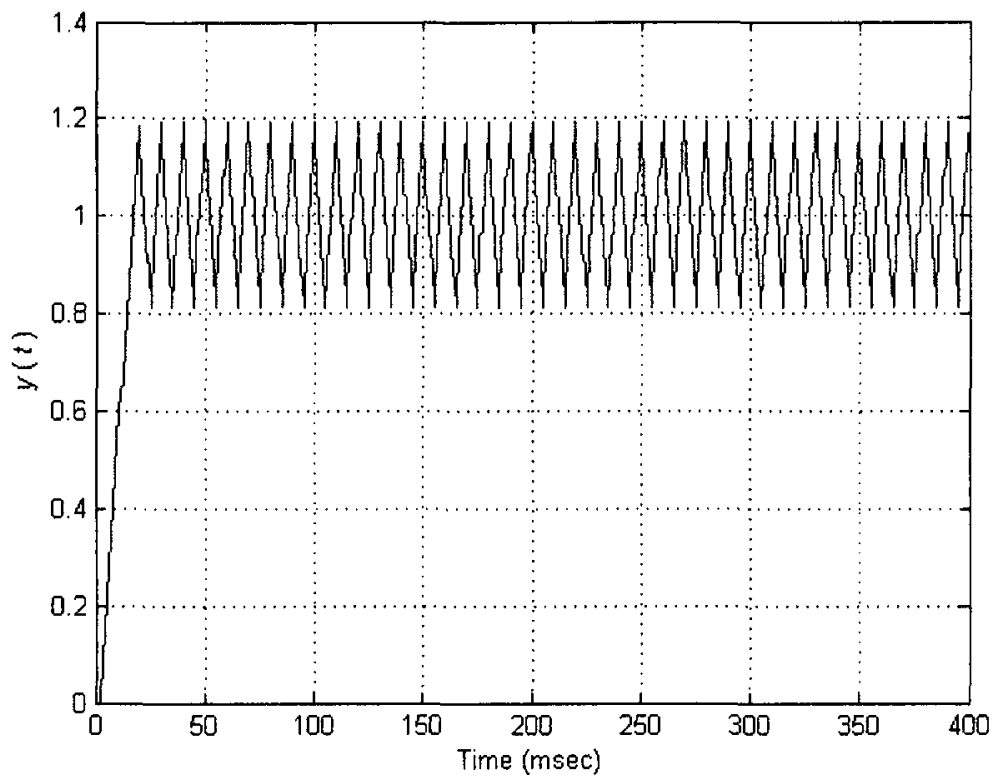

Figure 4.9: The step response of the IS-856 uplink using the control law (4.3) when $K=0.1$, and a two-level error comparison.

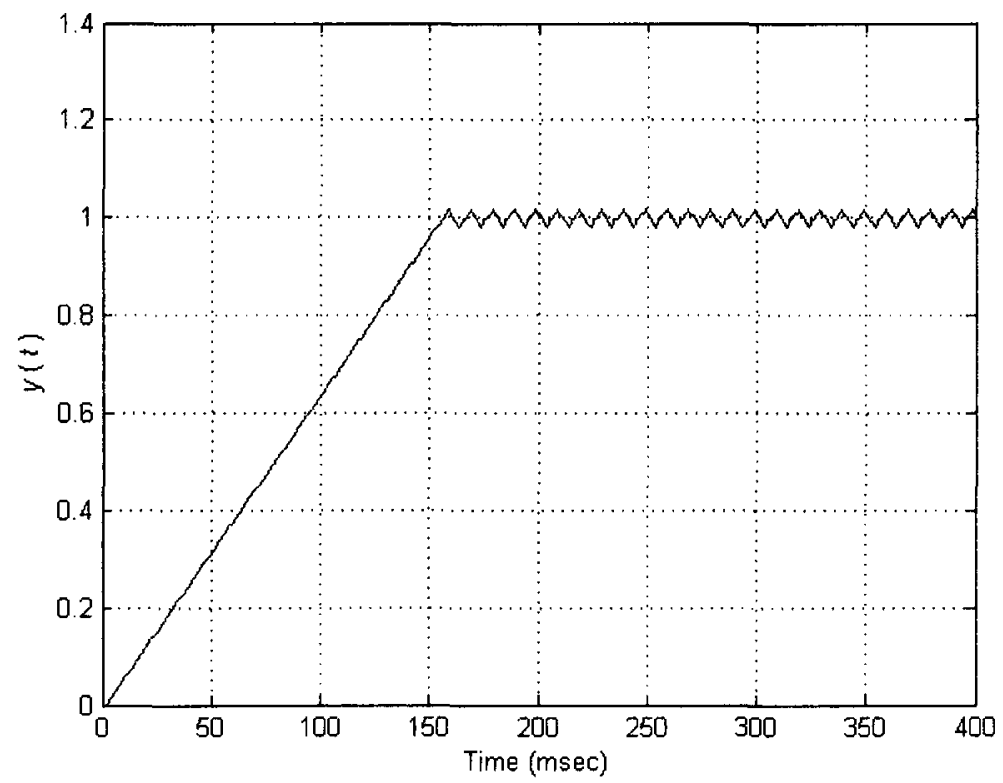

Figure 4.10: The step response of the IS-856 uplink using the linear control law (4.3) with $K=0.01$, and a two-level error comparison. 
Table 4.1: The intermediate points obtained by applying the BFGS optimization method to the underlying problem with the initial control parameters $z_{0}=0, p_{0}=0$ and $k=0$.

\begin{tabular}{|c|c|c|c|}
\hline Iteration & Func-count & $f(x)$ & Step-size \\
\hline 0 & 4 & 80200 & \\
\hline 1 & 16 & 863.585 & $1.55948-009$ \\
\hline 2 & 24 & 793.029 & 10 \\
\hline 3 & 28 & 780.323 & 1 \\
\hline 4 & 36 & 780.32 & 10 \\
\hline 5 & 40 & 780.312 & 1 \\
\hline 6 & 44 & 780.272 & 1 \\
\hline 7 & 48 & 780.186 & 1 \\
\hline 8 & 52 & 779.943 & 1 \\
\hline 9 & 56 & 779.335 & 1 \\
\hline 10 & 60 & 777.81 & 1 \\
\hline 11 & 64 & 774.451 & 1 \\
\hline 12 & 68 & 768.187 & 1 \\
\hline 13 & 72 & 755.884 & 1 \\
\hline 14 & 88 & 746.343 & 0.271 \\
\hline 15 & 100 & 733.435 & 0.19 \\
\hline 16 & 112 & 721.413 & 0.19 \\
\hline 17 & 124 & 708.72 & 0.19 \\
\hline
\end{tabular}

is required to choose initial parameters (which are often required to be stabilizing parameters). Choosing $p_{0}=0$ and $z_{0}=0$, the underlying iterative optimization method arrives at $z_{0}=-0.1276, p_{0}=0.1275$ and $K=0.0492$. The system output under this controller is depicted in Fig. 4.11, and the intermediate points in the optimization procedure are given in Table 4.1.

By setting the initial point to $z_{0}=-0.1, p_{0}=-0.7$ and $K=0.1$, the results in Table 4.2 are obtained. The iterative BFGS optimization procedure arrives at the optimal parameters $z_{0}=-0.1028, p_{0}=-0.7004$ and $K=0.0496$. The step response of the system under resultant controller is depicted in Fig. 4.12.

Using a three-level quantization in the AN, the output of the system regulated with this controller is depicted in Figs. 4.13 and 4.14 for $K=0.1$ and $K=0.5$, 


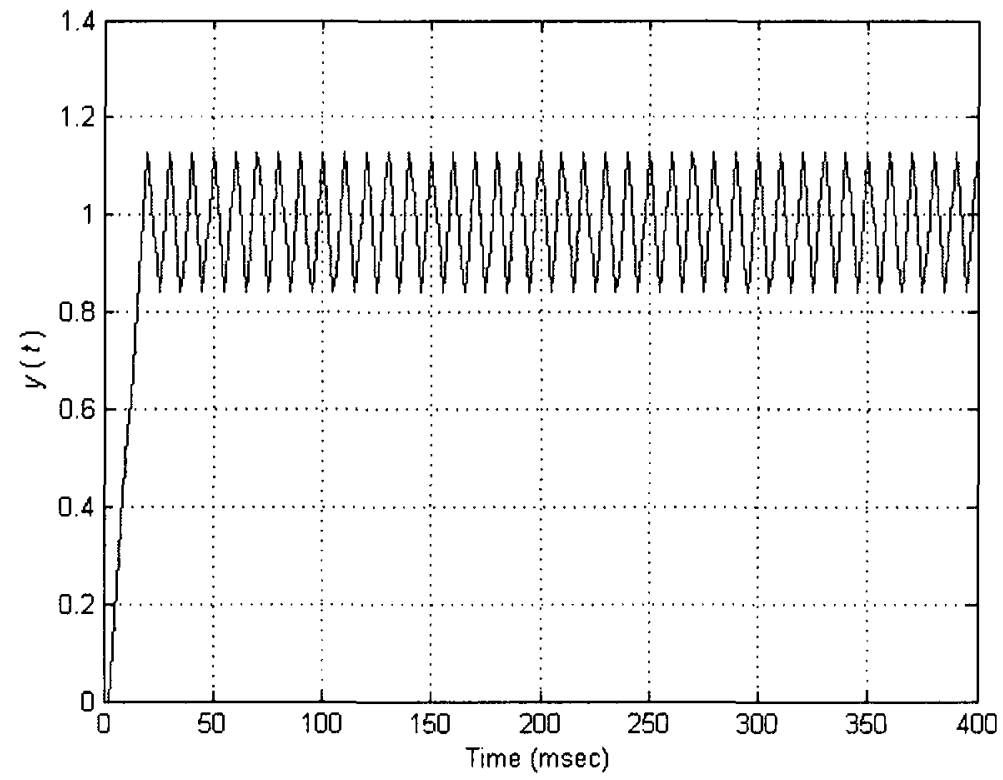

Figure 4.11: The step response of the IS-856 uplink using the optimal values obtained by applying the BFGS method with the initial parameters $z_{0}=0, p_{0}=0$ and $K=0$.

Table 4.2: The intermediate points obtained by applying the BFGS optimization method to the underlying problem with the initial control parameters $z_{0}=-0.1$, $p_{0}=-0.7$ and $K=0.1$.

\begin{tabular}{|c|c|c|c|}
\hline Iteration & Func-count & $f(x)$ & Step-size \\
\hline 0 & 4 & 923.911 & \\
\hline 1 & 16 & 261.139 & $2.31264-006$ \\
\hline 2 & 24 & 261.123 & 0.01 \\
\hline 3 & 28 & 261.1 & 1 \\
\hline 4 & 36 & 260.97 & 1 \\
\hline 5 & 40 & 260.716 & 1 \\
\hline
\end{tabular}




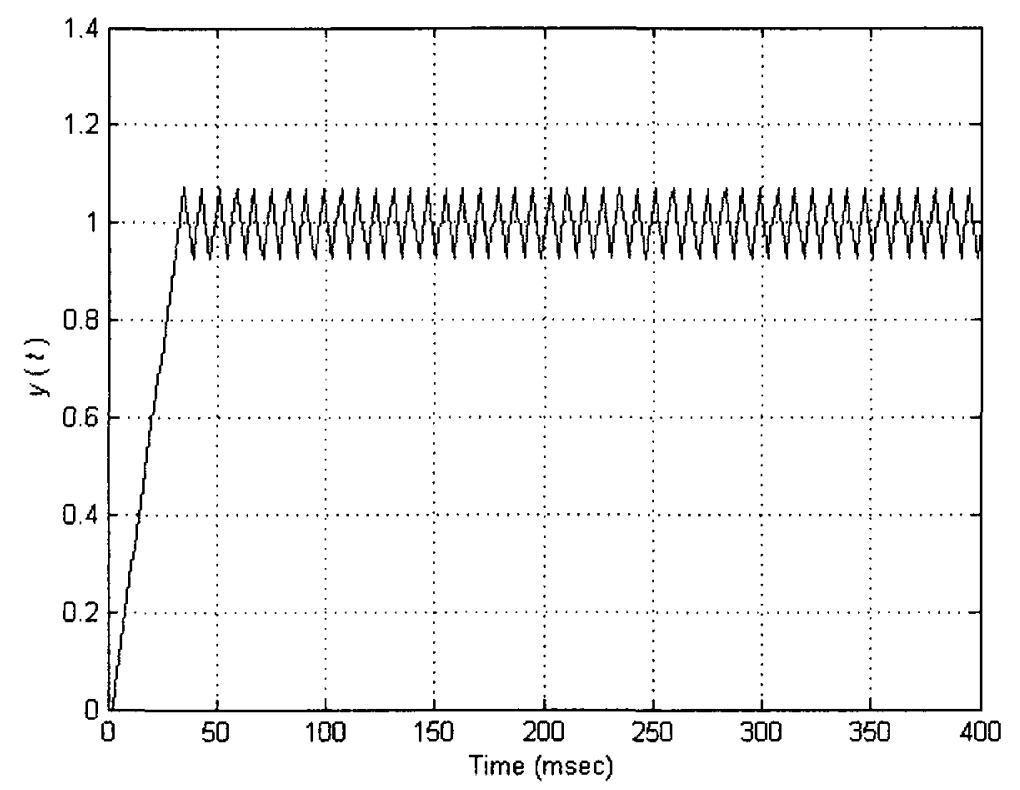

Figure 4.12: The step response of the IS-856 uplink, using the optimal values obtained by applying the BFGS method with the initial parameters $z_{0}=-0.1$, $p_{0}=-0.7$ and $K=0.1$.

respectively. It can be observed from these figures that using the control parameter $K>K_{\text {critical }}$ results in a stable limit cycle.

It can be verified that in the case of a three-level quantization after seven steps of optimization iteration, the local minimum for the performance index is found. The intermediate results are provided in Table 4.3, and the optimization procedure converges to the optimal control parameters $z_{0}=-0.0998, p_{0}=-0.7001$ and $K=0.1110$. The resultant network output under this controller is depicted in Fig. 4.15.

In the next step, the initial points for control parameters are set to certain values that result in high-amplitude limit cycle, e.g. $z_{0}=-0.1, p_{0}=-0.7$ and $K=1$. The intermediate results in Table 4.4 , and the procedure approaches a local minimum with control parameters $z_{0}=0.4781, p_{0}=-0.7990$ and $K=0.2027$ that is depicted in Fig. 4.16. It can be seen from this figure that under this optimal 


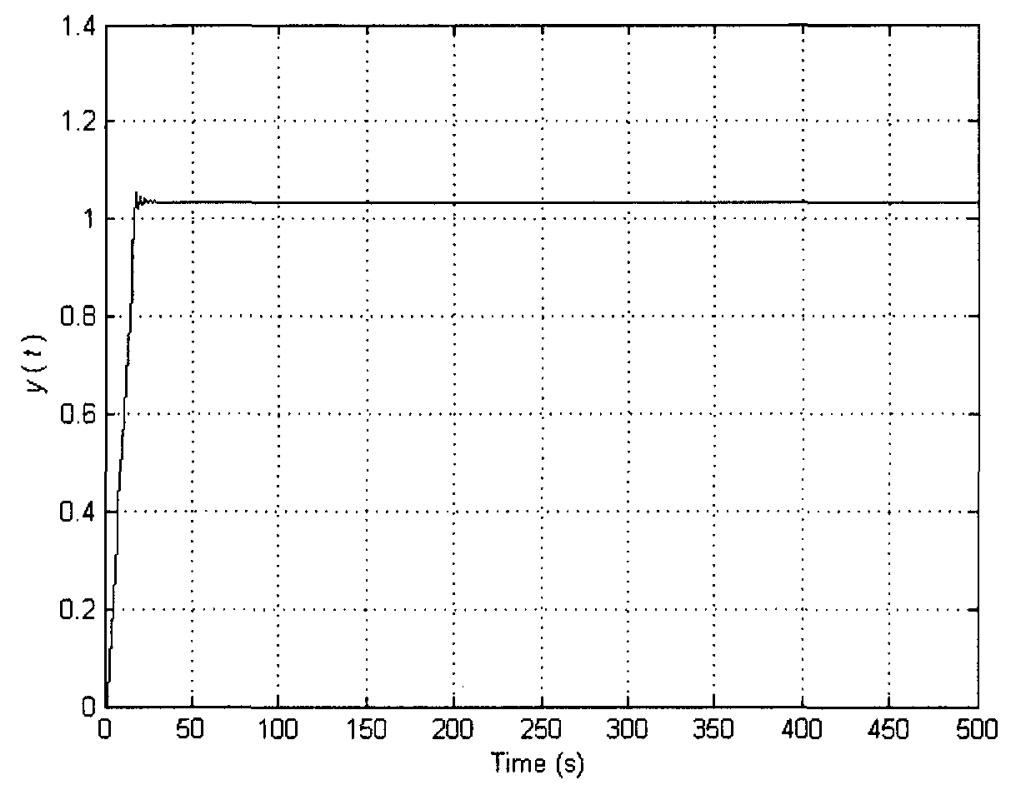

Figure 4.13: The step response of the IS-856 uplink using the linear control law (4.3) with $K=0.1$, and a three-level error comparison.

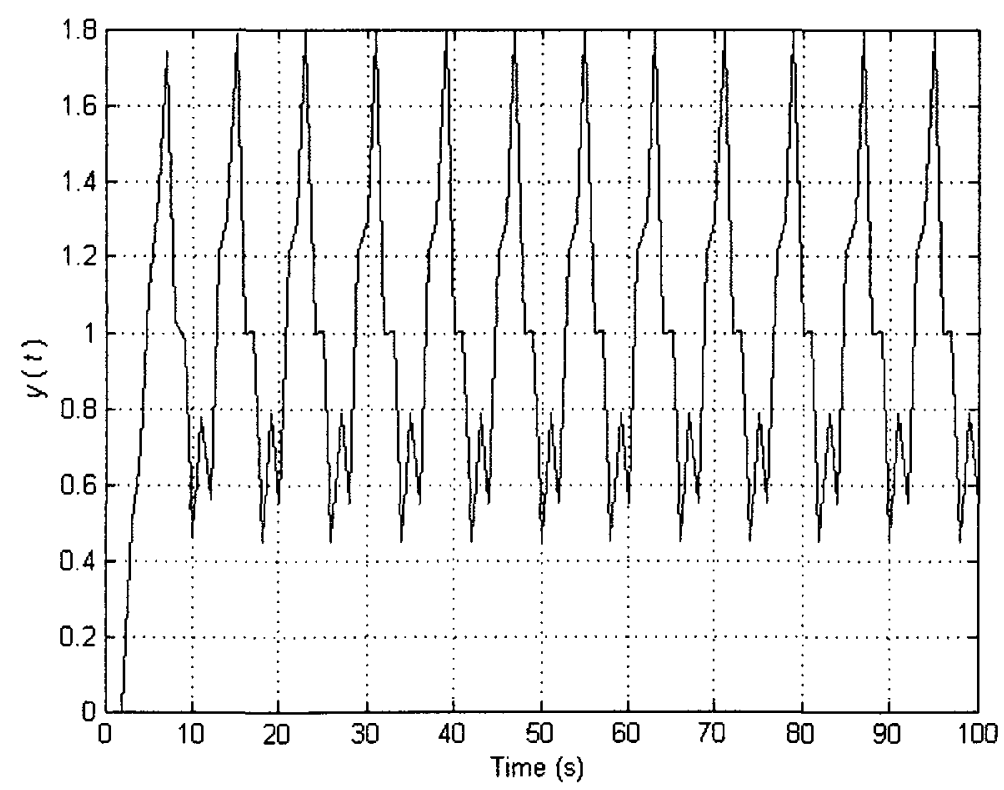

Figure 4.14: The step response of the IS-856 uplink, which includes a limit cycle as the control gain is greater than $K_{\text {critical }}$. 
Table 4.3: The intermediate points in the optimization used in Fig. 4.15.

\begin{tabular}{|c|c|c|c|}
\hline Iteration & Func-count & $f(x)$ & Step-size \\
\hline 0 & 4 & 125250 & \\
\hline 1 & 16 & 94.1572 & $2.1032 \mathrm{e}-009$ \\
\hline 2 & 24 & 52.0568 & 10 \\
\hline 3 & 32 & 48.4 & 0.1 \\
\hline 4 & 36 & 260.97 & 1 \\
\hline 5 & 56 & 39.5542 & 0.19 \\
\hline 6 & 68 & 37.2541 & 0.19 \\
\hline 7 & 84 & 36.3494 & 0.109 \\
\hline
\end{tabular}

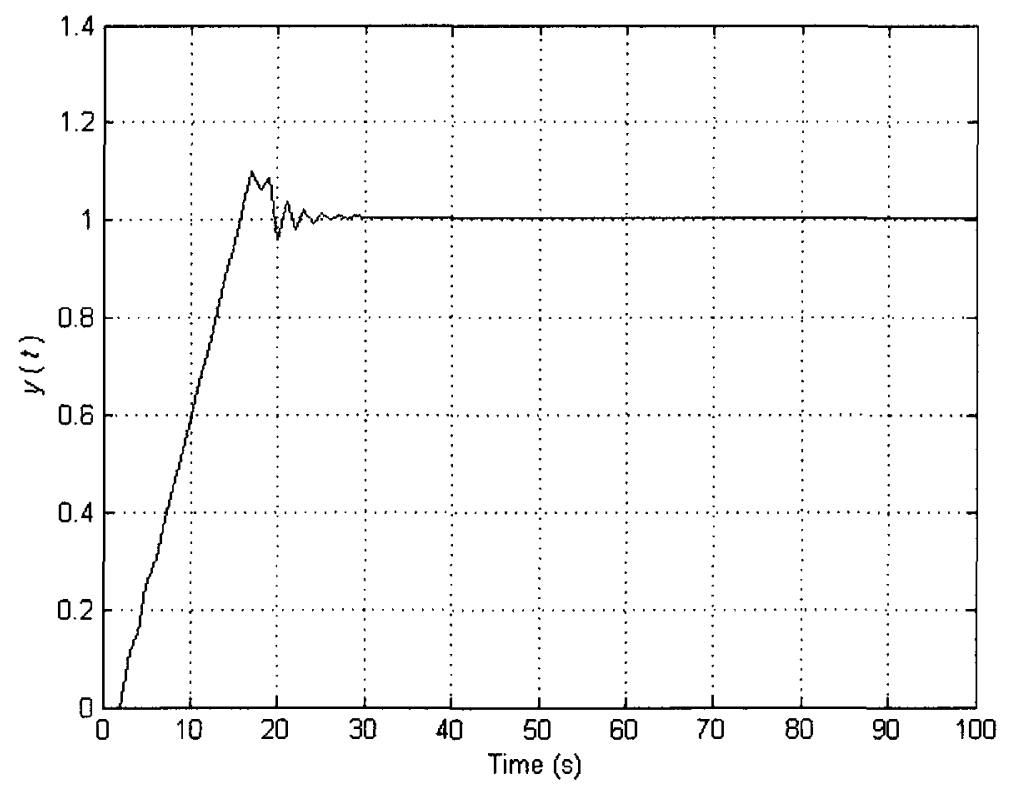

Figure 4.15: The step response of the IS- 856 uplink, using the optimal values obtained by applying the optimal controller given by $z_{0}=-0.0998, p_{0}=-0.7001$ and $K=0.111$. 
Table 4.4: The intermediate points in the optimization used in Fig. 4.16.

\begin{tabular}{|c|c|c|c|}
\hline Iteration & Func-count & $f(x)$ & Step-size \\
\hline 0 & 4 & 140162 & \\
\hline 1 & 16 & 637.123 & $3.28324 \mathrm{e}-006$ \\
\hline 2 & 24 & 336.14 & 0.1 \\
\hline 3 & 28 & 38.6611 & 1 \\
\hline 4 & 36 & 37.8516 & 0.190739 \\
\hline 5 & 52 & 37.4599 & 820 \\
\hline 6 & 56 & 37.22495 & 1 \\
\hline 7 & 64 & 37.0647 & 0.446584 \\
\hline 8 & 68 & 36.5922 & 1 \\
\hline 9 & 76 & 36.4555 & 0.465285 \\
\hline 10 & 80 & 36.1081 & 1 \\
\hline 11 & 84 & 35.8111 & 1 \\
\hline 12 & 100 & 35.4664 & 0.271 \\
\hline 13 & 120 & 35.4389 & 0.0271 \\
\hline
\end{tabular}

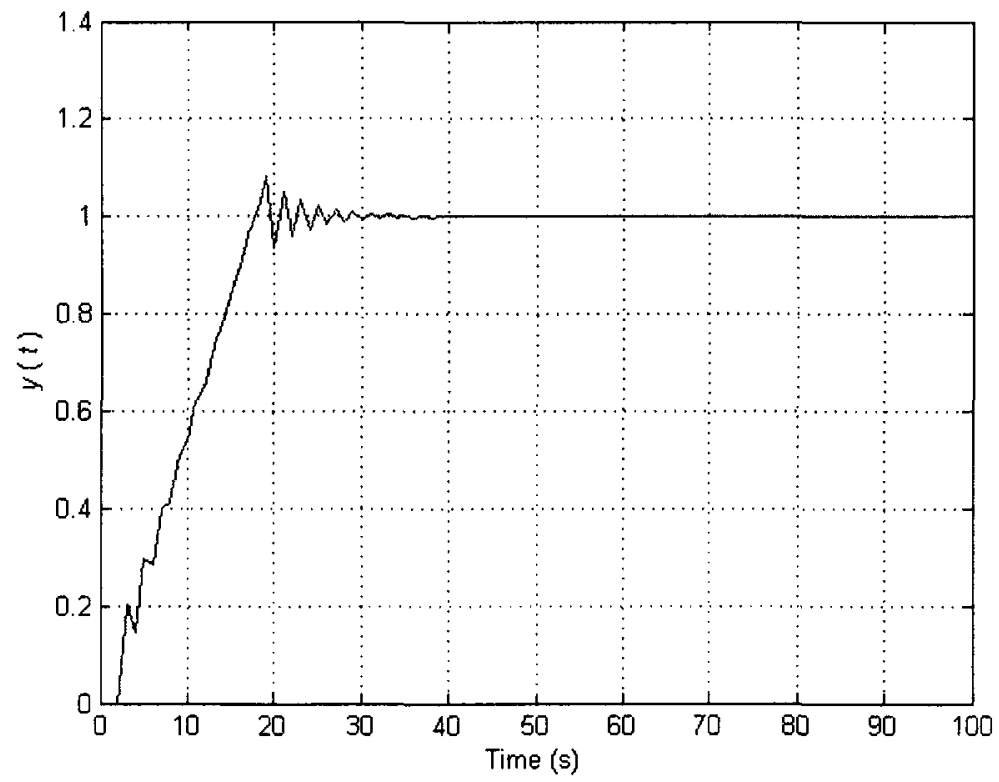

Figure 4.16: The step response of the IS-856 uplink, using the optimal values obtained by applying the BFGS method with the initial parameters $z_{0}=0.4781$, $p_{0}=-0.7990$ and $K=0.2027$. 
controller, the steady state error is negligible compared to the previous cases. 


\section{Chapter 5}

\section{Controller Design Using Adaptive Strategy: Stability Analysis}

\section{$5.1 \quad$ Introduction}

In this chapter, a nonlinear control framework is proposed for IS-856 uplink. The controller design technique is then introduced and the stability analysis for the resultant closed-loop system is presented. First, it will be assumed that the number of ATs in the network is constant $(n=N)$, and the controller is designed accordingly. Then, the controllers will be revisited to use a single-bit feedback (RAB) as defined in IS-856 standard. The stability analysis will be provided for the case when the number of ATs is subject to change $(n=N(t))$, using the theory of switched systems [15]. Finally, the controllers obtained will be modified properly to retain network stability and performance in the presence of time-delay and an upper-bound for the tracking error is obtained in the presence of time-delay in the control loop. The simulation results are presented to elucidate the effectiveness of the proposed approach. 


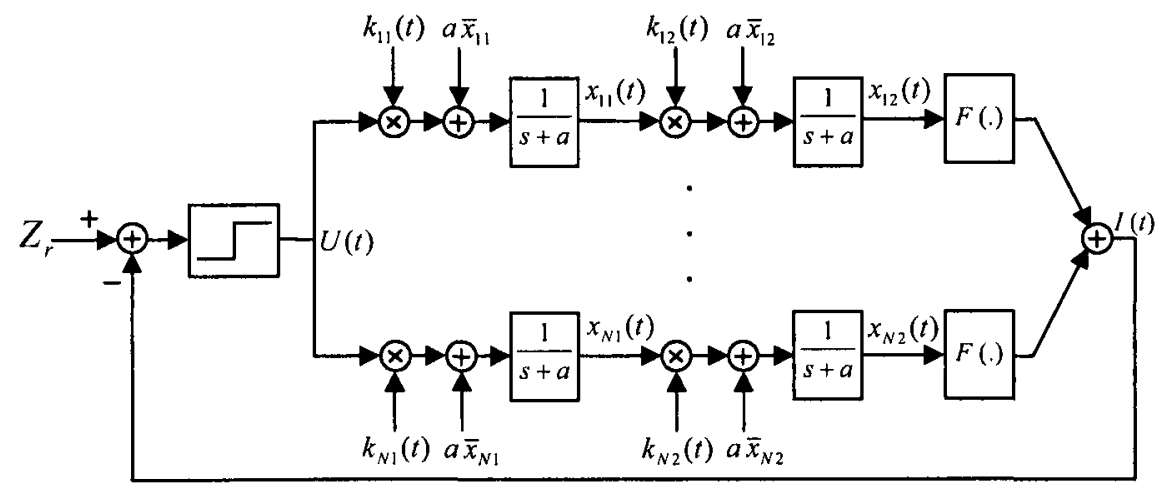

Figure 5.1: IS-856 uplink rate controller block diagram with the control law (5.1), for the case when the number of users is fixed.

\subsection{Controller Design for Fixed Number of Users}

Assume that $n$ (the number of ATs in the network) is constant; denote this constant number with $N$. Consider a control structure consisting of two simple first-order controllers with two time-varying coefficients $k_{i 1}(t)$ and $k_{i 2}(t)$ for the $i$-th AT, $i \in$ $\mathbf{N}:=\{1, \ldots, \mathbf{N}\}$. The closed-loop system model can be written as follows

$$
\begin{aligned}
& {\left[\begin{array}{c}
\dot{x}_{11}(t) \\
\dot{x}_{12}(t) \\
\vdots \\
\dot{x}_{N 1}(t) \\
\dot{x}_{N 2}(t)
\end{array}\right]=\left[\begin{array}{ccccc}
-a & 0 & \cdots & 0 & 0 \\
k_{12}(t) & -a & \cdots & 0 & 0 \\
\vdots & & \ddots & & \vdots \\
0 & 0 & \cdots & -a & 0 \\
0 & 0 & \cdots & k_{N 2}(t) & -a
\end{array}\right]\left[\begin{array}{c}
x_{11}(t) \\
x_{12}(t) \\
\vdots \\
x_{N 1}(t) \\
x_{N 2}(t)
\end{array}\right]} \\
& +\left[\begin{array}{c}
a \bar{x}_{11}+k_{11}(t) U(t) \\
a \bar{x}_{12} \\
\vdots \\
a \bar{x}_{N 1}+k_{N 1}(t) U(t) \\
a \bar{x}_{N 2}
\end{array}\right] \\
& U(t)=\operatorname{sgn}\left(Z_{r}-y(t)\right) \\
& y(t)=\frac{I(t)}{P_{\text {pilot }}}
\end{aligned}
$$


where $a$ is a strictly positive scalar. The structure of the controller introduced above is depicted in Fig. 5.1.

It is desired that the network output $y(t)$ in the closed-loop model (5.1c) reaches a sufficiently close neighborhood of $Z_{r}$. In other words, the objective here is to ensure the convergence of the state vector $\left[x_{11}(t) x_{12}(t) \cdots x_{N 1}(t) x_{N 2}(t)\right]^{T}$ to a nominal state $\left[\begin{array}{lllll}\bar{x}_{11} & \bar{x}_{12} & \cdots & \bar{x}_{N 1} & \bar{x}_{N 2}\end{array}\right]^{T}$ which is, in fact, an equilibrium point for the network, and is assumed to lie in a desired region. This nominal state is chosen based on the reference ROT and the number of active users. The dynamic equations of the time-varying control coefficients $k_{i 1}(t)$ and $k_{i 2}(t)$ are designed as follows

$$
\begin{aligned}
& \dot{k}_{i 1}(t)=-a k_{i 1}(t)-U(t) x_{i 1}(t)+U(t) \bar{x}_{i 1} \\
& \dot{k}_{i 2}(t)=-a k_{i 2}(t)-x_{i 1}(t) x_{i 2}(t)+x_{i 1}(t) \bar{x}_{i 2}
\end{aligned}
$$

for any $i \in \mathbf{N}$, to guarantee the stability of the network as will be seen in Theorem 1 . Define the new state vector $Y(t)$ as follows

$$
Y(t)=\left[\begin{array}{c}
y_{11}(t) \\
y_{12}(t) \\
\vdots \\
y_{N 1}(t) \\
y_{N 2}(t)
\end{array}\right]:=\left[\begin{array}{c}
x_{11}(t)-\bar{x}_{11} \\
x_{12}(t)-\bar{x}_{12} \\
\vdots \\
x_{N 1}(t)-\bar{x}_{N 1} \\
x_{N 2}(t)-\bar{x}_{N 2}
\end{array}\right]
$$

Define also the matrices $A(t)$ and $B(t)$ as

$$
A(t)=\left[\begin{array}{ccccc}
-a & 0 & \cdots & 0 & 0 \\
k_{12}(t) & -a & \cdots & 0 & 0 \\
\vdots & & \ddots & & \vdots \\
0 & 0 & \cdots & -a & 0 \\
0 & 0 & \cdots & k_{N 2}(t) & -a
\end{array}\right]
$$




$$
B(t)=\left[\begin{array}{c}
k_{11}(t) U(t) \\
\bar{x}_{11} k_{12}(t) \\
\vdots \\
k_{N 1}(t) U(t) \\
\bar{x}_{N 1} k_{N 2}(t)
\end{array}\right]
$$

The state-space model (5.1) can now be expressed in the new coordinates as

$$
\dot{Y}(t)=A(t) Y(t)+B(t)
$$

and the dynamic equations of the time-varying coefficients (5.2) can then be simplified to

$$
\begin{aligned}
& \dot{k}_{i 1}(t)=-a k_{i 1}(t)-U(t) y_{i 1}(t) \\
& \dot{k}_{i 2}(t)=-a k_{i 2}(t)-y_{i 1}(t) y_{i 2}(t)-\bar{x}_{i 1} y_{i 2}(t)
\end{aligned}
$$

for all $i \in \mathbf{N}$.

The following theorem provides a sufficient condition for the stability of the system (5.6).

Theorem 1. The state trajectories of system (5.6) with the time-varying control coefficients $k_{i 1}(t)$ and $k_{i 2}(t)$ given in (5.7) are globally exponentially stable in the sense of Lyapunov.

Proof: To carry out the stability analysis, choose the Lyapunov function candidate as

$$
V(t)=\frac{1}{2} Y^{T}(t) Y(t)+\frac{1}{2} \sum_{i=1}^{N} k_{i 1}^{2}(t)+\frac{1}{2} \sum_{i=1}^{N} k_{i 2}^{2}(t)
$$

The derivative of this Lyapunov function along with the state trajectories of system (5.6) yields

$$
\begin{aligned}
\dot{V}(t)= & \frac{1}{2}\left(\dot{Y}^{T}(t) Y(t)+Y^{T}(t) \dot{Y}(t)\right) \\
& +\sum_{i=1}^{N} \dot{k}_{i 1}(t) k_{i 1}(t)+\sum_{i=1}^{N} \dot{k}_{i 2}(t) k_{i 2}(t)
\end{aligned}
$$


Substituting (5.6) in (5.9) yields

$$
\begin{aligned}
\dot{V}(t)= & -a y_{11}^{2}(t)+k_{11}(t) U(t) y_{11}(t)+k_{12}(t) y_{11}(t) y_{12}(t) \\
& -a y_{12}^{2}(t)+\bar{x}_{11} k_{12}(t) y_{12}(t)+\cdots \\
& -a y_{N 1}^{2}+k_{N 1} U(t) y_{N 1}(t)+k_{N 2}(t) y_{N 1}(t) y_{N 2}(t) \\
& -a y_{N 2}^{2}+\bar{x}_{N 1} k_{N 2}(t) y_{n 2}(t) \\
& +\sum_{i=1}^{N} \dot{k}_{i 1}(t) k_{i 1}(t)+\sum_{i=1}^{N} \dot{k}_{i 2}(t) k_{i 2}(t)
\end{aligned}
$$

Now, using (5.7) one will arrive at the following relation

$$
\begin{aligned}
\dot{V}(t)= & -a \sum_{i=1}^{N} y_{i 1}^{2}(t)-a \sum_{i=1}^{N} y_{i 2}^{2}(t) \\
& -a \sum_{i=1}^{N} k_{i 1}^{2}(t)-a \sum_{i=1}^{N} k_{i 2}^{2}(t)
\end{aligned}
$$

It can be concluded from (5.11) that

$$
\dot{V}(t)=-2 a V(t)
$$

which guarantees the negative definiteness of the derivative function $\dot{V}(t)$ (note that $a$ is strictly positive, as mentioned earlier). This means that the origin is an equilibrium point for the system (5.6), and that this equilibrium point is globally exponentially stable.

Remark 4. The stability of system (5.1) follows directly from the stability of system (5.6), and hence the state trajectories of system (5.1) converge exponentially to $\left[\begin{array}{llll}\bar{x}_{11} & \cdots & \bar{x}_{N 1} & \bar{x}_{N 2}\end{array}\right]^{T}$.

In order to achieve exact tracking, the trajectories of $x_{i 2}(t), \forall i \in \mathbf{N}$ should be such that $y(t)=Z_{r}$ for some $t$. Therefore, $\bar{x}_{i 2}$ should be defined in such a way that $\tilde{F}\left(\bar{x}_{i 2}\right)=\frac{Z_{r}}{N}, \forall i \in \mathbf{N}$. Define the tracking error similarly to (3.6)

$$
e(t)=\left(Z_{r}-\sum_{i=1}^{N(t)} \tilde{F}\left(x_{i 2}(t)\right)\right)
$$


Since $\Lambda$ is a finite set consisting of specific values only, it is not always possible to find a value for $\bar{x}_{i 2}$ in this set, for which $\tilde{F}\left(\bar{x}_{i 2}\right)$ is exactly equal to $\frac{Z_{r}}{N(t)}$. In order to overcome this hurdle, it is desired to fit the points in the mapping (3.1) with first and second-order continuous functions with appropriate uncertainty bounds, to cover the whole mapping $\tilde{F}(\cdot)$. Moreover a probabilistic dynamic rate selection scheme method [11] is also studied.

\subsubsection{First-Order Approximation for the Mapping $\tilde{F}(\cdot)$}

For the case when the number of ATs is fixed, one can fit the closest line of the form

$$
\tilde{F}_{1}\left(x_{i 2}(t)\right)=\omega_{1} x_{i 2}(t)+\omega_{0}
$$

to the points in Fig. 3.1. The optimal coefficients (in the sense of least mean squares) are given by $\omega_{1}=0.2698$ and $\omega_{0}=-2.2958$. One can write

$$
\tilde{F}\left(x_{i 2}(t)\right)=\tilde{F}_{1}\left(x_{i 2}(t)\right)+\delta\left(x_{i 2}(t)\right)
$$

where $\delta\left(x_{i 2}(t)\right)$ is the approximation error whose maximum magnitude is denoted by $\delta_{m 1}$ (i.e., $\left.\left|\delta\left(x_{i 2}(t)\right)\right| \leq \delta_{m 1}\right)$. The first order approximation $\tilde{F}_{1}$ is depicted in Fig. 5.2 , along with the original nonuniform quantizer $\tilde{F}$, and two lines parallel to the resultant first-order approximation, which enclose $\tilde{F}$. These lines are obtained by shifting the resultant first-order approximation up and down by a value less than or equal to $\delta_{m 1}$.

Now, by substituting $\tilde{F}_{1}\left(x_{i 2}(t)\right)$ with $\frac{Z_{r}}{N}$ (when $N$ users are active), $\bar{x}_{i 2}$ can be approximated as

$$
\bar{x}_{i 2}=\frac{\frac{Z_{r}}{N}-\omega_{0}}{\omega_{1}}
$$

Using the above formula, the maximum magnitude of the output error (5.13) in steady state can be found as

$$
\max |e(t)|=\left|Z_{r}-N \tilde{F}_{1}\left(\bar{x}_{i 2}\right)\right|=N \delta_{m 1}
$$




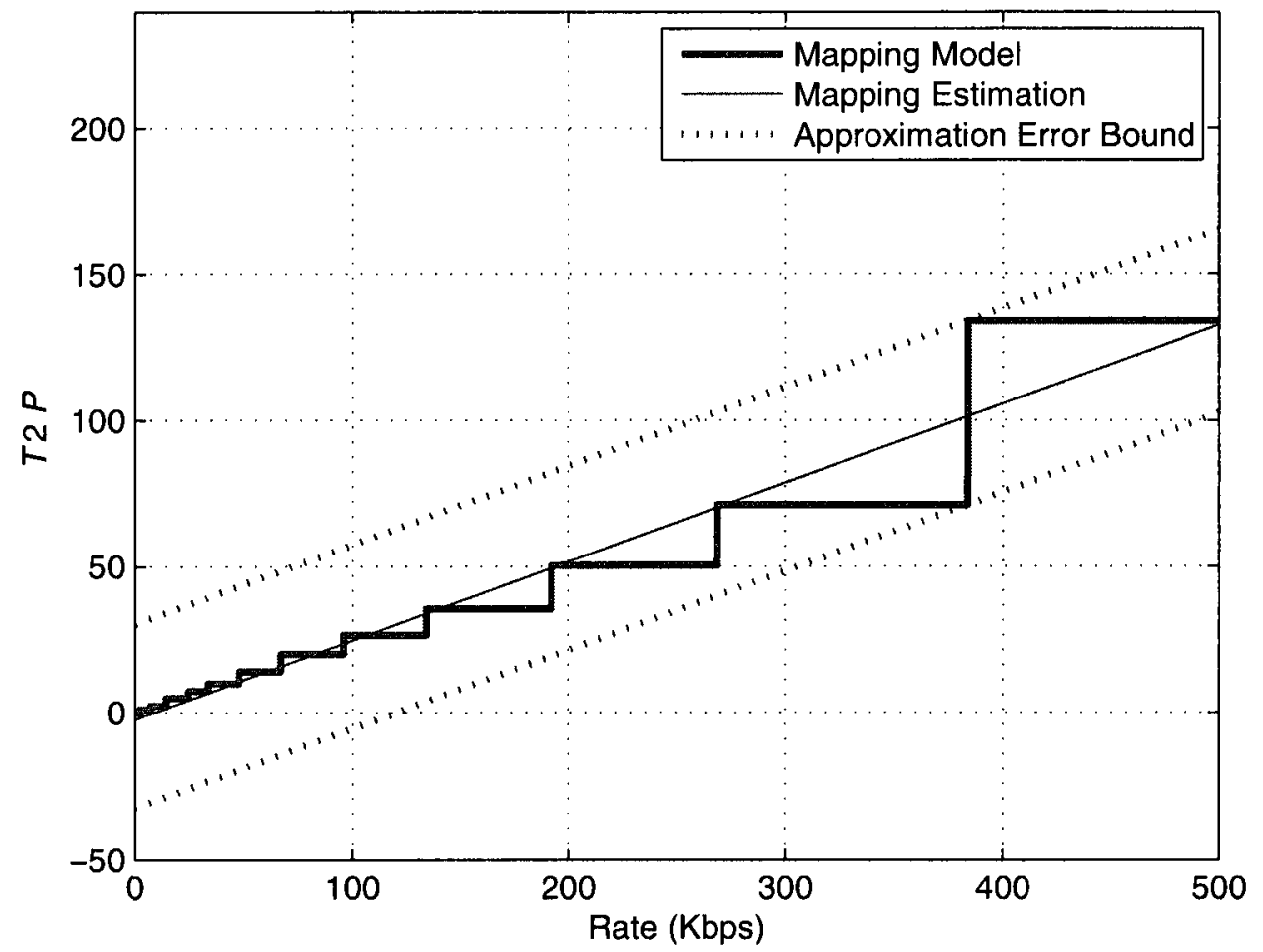

Figure 5.2: Linear approximation of the piecewise constant function $\tilde{F}(\cdot)$ as well as two boundary lines containing the function. 


\subsubsection{Second-Order Approximation for the Mapping $\tilde{F}(\cdot)$}

The points in Fig. 3.1 can alternatively be fitted by a second-order polynomial (to attain a higher precision). For the case when the number of ATs is fixed, this results in

$$
\tilde{F}_{2}\left(x_{i 2}(t)\right)=\nu_{2} x_{i 2}^{2}(t)+\nu_{1} x_{i 2}(t)+\nu_{0}+\delta_{2}\left(x_{i 2}(t)\right)
$$

Analogously to the first-order case, the optimal coefficients are obtained as $\nu_{2}=$ $0.0003, \nu_{1}=0.1516$, and $\nu_{0}=3.034$. In addition, the approximation error $\delta_{2}\left(x_{i 2}(t)\right)$ in this case (which is defined similarly to the first-order approximation) has an upperbound equal to $\delta_{m 2}$. This bound is smaller than $\delta_{m 1}$ corresponding to the first-order case, as expected. The result of this approximation along with the second-order upper and lower bounds for $\tilde{F}(\cdot)$ parallel to $\tilde{F}_{2}(\cdot)$ are shown in Fig. 5.3. Now, similar to the development presented in the first-order approximation, $\bar{x}_{i 2}$ can be approximated by

$$
\bar{x}_{i 2}=\tilde{F}_{2}^{-1}\left(\frac{Z_{r}}{N}\right)
$$

and consequently in steady state the maximum bound on the error can be found as follows

$$
\begin{aligned}
\max |e(t)| & =\left|Z_{r}-N \tilde{F}_{2}\left(x_{i 2_{0}}\right)\right| \\
& =N \delta_{m 2}
\end{aligned}
$$

Remark 5. It is to be noted that for a more accurate approximation of $\tilde{F}$ in (5.14) and (5.18), one can take into account the amount of existent traffic of the network. For example, in peak hours, the mapping should be approximated more accurately near the points in the set $\Gamma$, which are closer to the origin i.e. feasible rates close to the origin in the nonuniform quantizer represents higher traffic and should normally be approximated more precisely in peak times and should have stronger impact on the curves being sought. Furthermore, one can consider a probability density function (pdf) for the data rate in order to characterize the relative importance of 


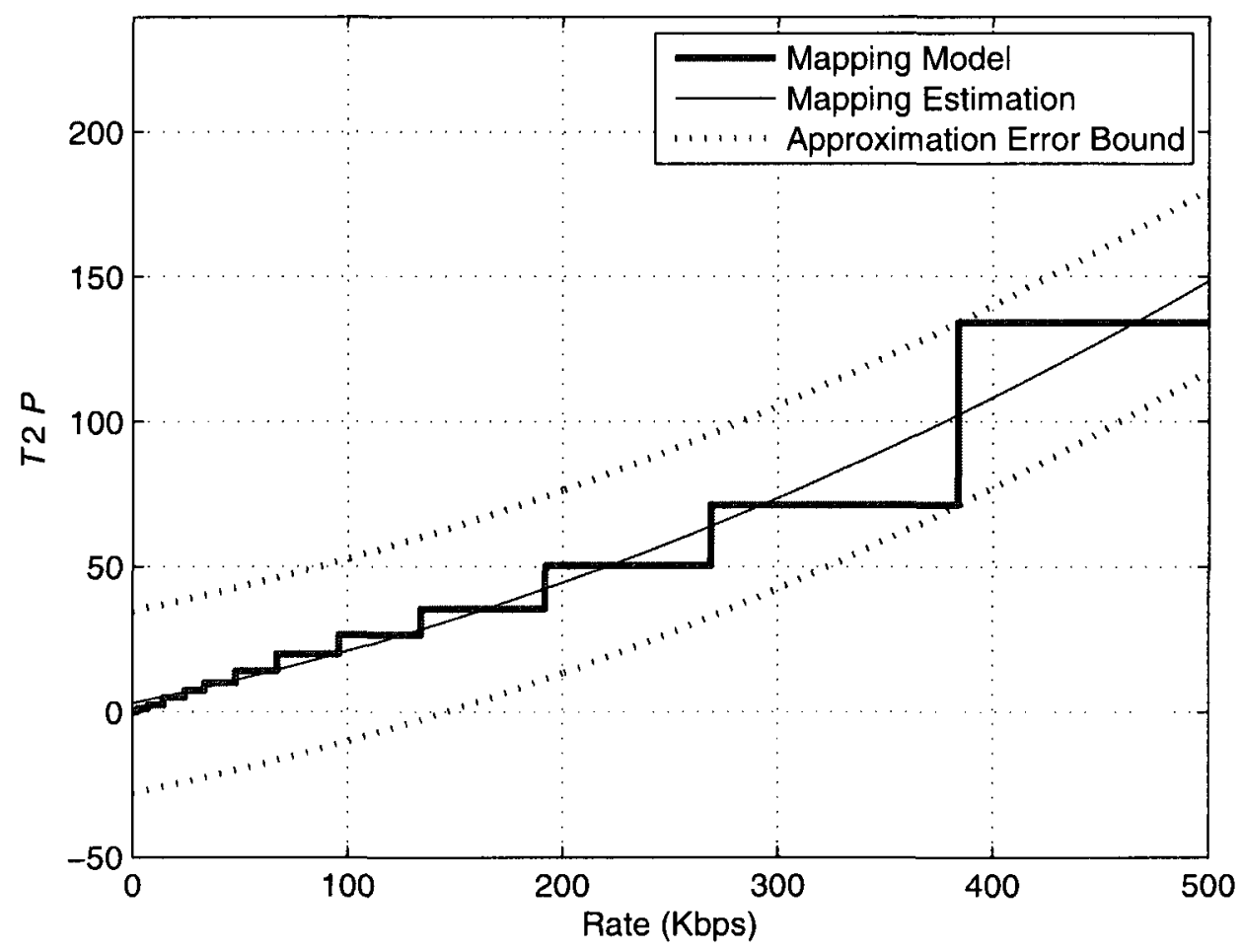

Figure 5.3: A second-order curve as the continuous approximation for the piecewise constant mapping $\tilde{F}(\cdot)$, and second-order curves for its upper and lower-bounds. 
different rates (in terms of how frequently they are being assigned in the network). This pdf can then be used as a weighting function in deriving the coefficients of the corresponding curves.

\subsubsection{Dynamic Rate Selection}

An online dynamic allocation algorithm is proposed now for ATs to dynamically adjust their equilibrium points $\left(\bar{x}_{i 2}\right)$. The steady-state error is calculated like previous methods. In this method each AT keeps calculating $\frac{Z_{r}}{N(t)}$ and the two levels of the Mapping $\tilde{F}(\cdot)$ that surround $\frac{Z_{r}}{N(t)}$, continuously. Supposing that at the time $t^{\prime}$ there are $N^{\prime}$ active users available in the network and the distances between $\frac{Z_{r}}{N^{\prime}}$ and the lower level and the upper level are $d_{N^{\prime} 1}$ and $d_{N^{\prime} 1}$ accordingly, and also suppose that the feasible rates associated with these two levels are $R_{1}$ and $R_{2}$ accordingly. If $d_{N^{\prime} 1}$ is smaller than $d_{N^{\prime} 2}$, the feasible rate $\bar{x}_{i 2}=R_{1}$ is selected as the equilibrium point for the $i^{\text {th }}$ AT, otherwise $\bar{x}_{i 2}=R_{2}$ is chosen.

$$
\left\{\begin{array}{l}
\bar{x}_{i 2}=R_{1}, \quad d_{N^{\prime} 1} \leq d_{N^{\prime} 2} \\
\bar{x}_{i 2}=R_{2}, \quad d_{N^{\prime} 1}>d_{N^{\prime} 2}
\end{array}\right.
$$

It can be shown that using this method the error (5.13) is as follows

$$
\left|e_{N^{\prime}}\right|=N^{\prime} \min \left\{d_{N^{\prime} 1}, d_{N^{\prime} 2}\right\}
$$

In Fig. 5.4, the magnitude of the steady-state error is shown for a network with up to 50 active full-buffered users and the three proposed methods for $\bar{x}_{i 2}$ provided by $(5.16),(5.19)$ and $(5.21)$. It can be observed from this figure that as the number of users increases, the second-order approximation results in a smaller error, and hence a more desirable behavior compared to first-order approximation. Moreover, the dynamic allocation's error is always equal or smaller than using first-order and second-order approximations. It is to be noted that the error depicted in Fig. 5.4 is, in fact, the overall error of all users; in other words, the average error of each user is roughly $N(t)$ times smaller than the overall error. 


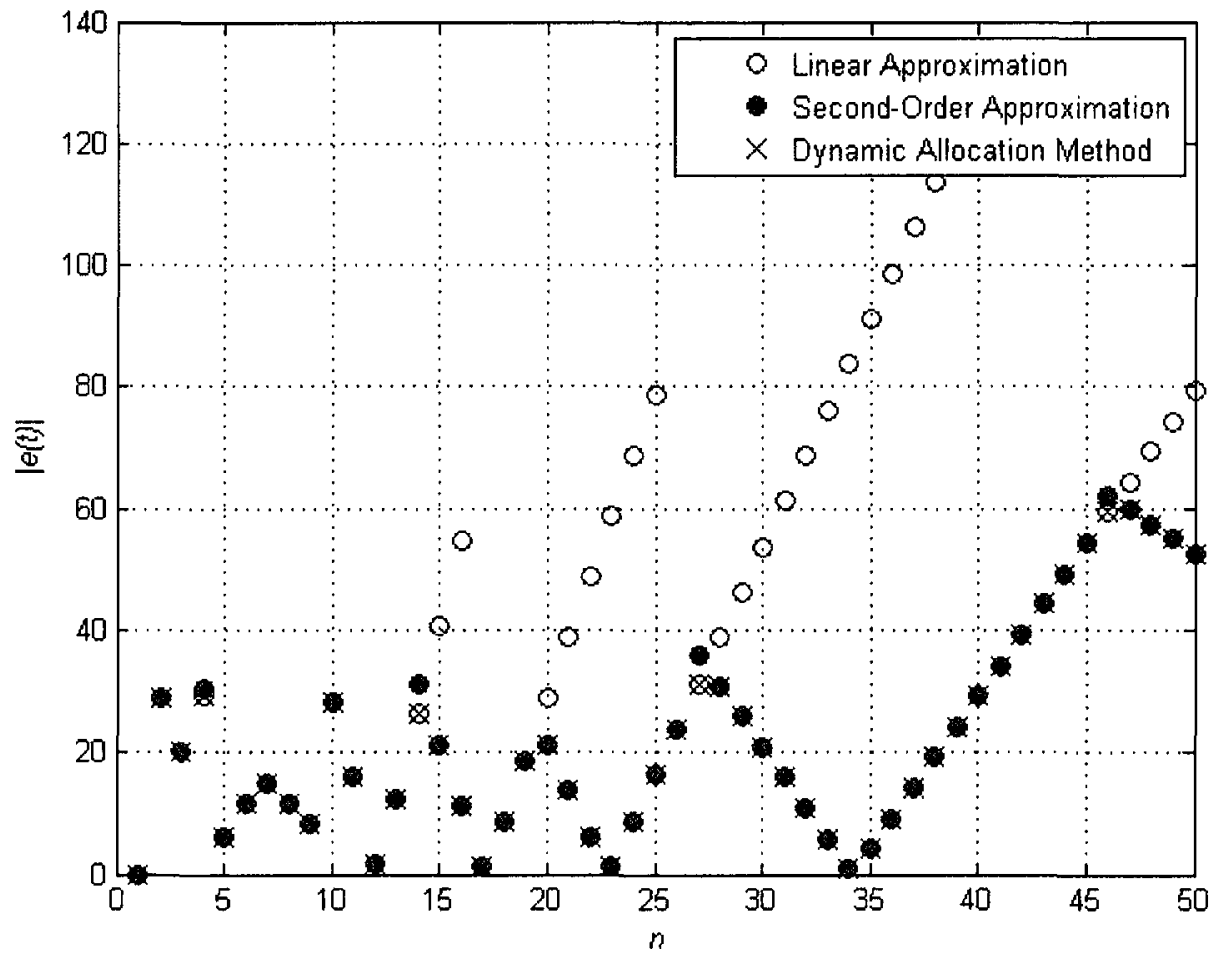

Figure 5.4: Magnitude of the error produced when the first-order approximation, second-order approximation, and dynamic allocation method are used for $T 2 P$ in a cell versus number of available active users. 


\subsubsection{Probabilistic Rate Selection}

If the output of the controller of the $i$-th AT at time $t_{0}$ is between two feasible rates $R_{1}$ and $R_{2}$, i.e.

$$
R_{1}<x_{i 2}\left(t_{0}\right)<R_{2}
$$

then this AT transmits data at the rate $R_{1}$, with the probability $p_{1}$ given by

$$
p_{1}=\frac{F_{k}\left(R_{2}\right)-\tilde{F}_{k}\left(x_{i 2}\left(t_{0}\right)\right)}{F_{k}\left(R_{2}\right)-F_{k}\left(R_{1}\right)}
$$

or at the feasible rate $R_{2}$, with the probability $p_{2}$ given below

$$
p_{2}=\frac{\tilde{F}_{k}\left(x_{i 2}\left(t_{0}\right)\right)-F_{k}\left(R_{1}\right)}{F_{k}\left(R_{2}\right)-F_{k}\left(R_{1}\right)}
$$

Note that $\tilde{F}_{k}($.$) in (5.24) and (5.25) is, in fact, a proper approximation of the map-$ ping $\tilde{F}(\cdot)$ from the feasible rates to feasible $T 2 P$, which can be the first-order approximation $\tilde{F}_{1}$, or the second-order one $\tilde{F}_{2}$.

\subsection{Controller Design for Unknown-fixed Num- ber of Users}

Let $N$ be the maximum number of ATs in a cell. Like previous section the control structure in Fig. 5.1 with two time varying coefficients $k_{i 1}(t), k_{i 2}(t)$ for the $i$-th AT, $i \in \mathbf{N}$ is considered. The state space representation for the closed-loop system is shown in (5.1). The objective is to ensure the convergence of the state vector $\left[x_{11}(t) x_{12}(t) \cdots x_{N 1}(t) x_{N 2}(t)\right]^{T}$ to a nominal state $\left[\bar{x}_{11}(t) \bar{x}_{12}(t) \cdots \bar{x}_{N 1}(t) \bar{x}_{N 2}(t)\right]^{T}$ which is, in fact, an equilibrium point for the network (which corresponds to the perfect regulation of ROT). This nominal state is chosen only based on the common single-bit signal (3.7) received from $\mathrm{AN}$ as follows

$$
\dot{\bar{x}}_{i 2}(t)=U(t)
$$


for all $i \in \mathbf{N}$. For simplicity and with no loss of generality, assume that

$$
\bar{x}_{i 1}(t)=0
$$

(notice that the above assumption does not limit the scope of the results). The dynamic evolution of the time-varying coefficients $k_{i 1}(t)$ and $k_{i 2}(t)$ is given by (5.2). Define the new state vector $Y(t)$

$$
Y(t)=\left[\begin{array}{c}
y_{11}(t) \\
y_{12}(t) \\
\vdots \\
y_{N 1}(t) \\
y_{N 2}(t)
\end{array}\right]:=\left[\begin{array}{c}
x_{11}(t)-\bar{x}_{11} \\
x_{12}(t)-\bar{x}_{12} \\
\vdots \\
x_{N 1}(t)-\bar{x}_{N 1} \\
x_{N 2}(t)-\bar{x}_{N 2}
\end{array}\right]
$$

The state-space representation (5.1) in the new coordinates can be obtained as follows

$$
\dot{Y}(t)=A(t) Y(t)+B(t)
$$

where and $B(t)$ is defined as

$$
B(t)=\left[\begin{array}{c}
k_{11}(t) U(t) \\
-U(t) \\
\vdots \\
k_{N 1}(t) U(t) \\
-U(t)
\end{array}\right]
$$

It can be easily verified that the origin is the equilibrium point in the new coordinates. Note that using (5.28), the adaptive gains $k_{i 1}(t)$ and $k_{i 2}(t)$ can be rewritten as follows

$$
\begin{gathered}
\dot{k}_{i 1}(t)=-a k_{i 1}(t)-U(t) y_{i 1}(t) \\
\dot{k}_{i 2}(t)=-a k_{i 2}(t)-y_{i 1}(t) y_{i 2}(t)
\end{gathered}
$$

for all $i \in \mathbf{N}$. 
The following theorem states that the state of the system converges, and eventually remains in a certain neighborhood of the equilibrium.

Theorem 2. The state trajectories of system (5.29) along with the time-varying coefficients (5.31) are globally ultimately stable in the sense of Lyapunov.

Proof: Choose the same Lyapunov function like (5.8) The derivative of (5.8) is given by

$$
\begin{aligned}
\dot{V}(t)= & \frac{1}{2}\left(\dot{Y}^{T}(t) Y(t)+Y^{T}(t) \dot{Y}(t)\right) \\
& +\sum_{i=1}^{N} \dot{k}_{i 1}(t) k_{i 1}(t)+\sum_{i=1}^{N} \dot{k}_{i 2}(t) k_{i 2}(t)
\end{aligned}
$$

Substituting (5.29) in (5.32) yields

$$
\begin{aligned}
\dot{V}(t)= & -a y_{11}^{2}(t)+k_{11}(t) U(t) y_{11}(t)+k_{12}(t) y_{11}(t) y_{12}(t) \\
& -a y_{12}^{2}(t)-U(t)-a y_{N 1}^{2} \cdots \\
& +k_{N 1} U(t) y_{N 1}(t)+k_{N 2}(t) y_{N 1}(t) y_{N 2}(t)-a y_{N 2}^{2} \\
& +\sum_{i=1}^{N} \dot{k}_{i 1}(t) k_{i 1}(t)+\sum_{i=1}^{N} \dot{k}_{i 2}(t) k_{i 2}(t)
\end{aligned}
$$

Now, using (5.31) one will arrive at the following relation

$$
\begin{aligned}
\dot{V}(t)= & -a \sum_{i=1}^{N} y_{i 1}^{2}(t)-a \sum_{i=1}^{N} k_{i 1}^{2}(t)-a \sum_{i=1}^{N} k_{i 2}^{2}(t) \\
& -\sum_{i=1}^{N}\left(\sqrt{a} y_{i 2}(t)-\frac{U(t)}{2 \sqrt{a}}\right)^{2}+\frac{U(t)^{2}}{4 a} N
\end{aligned}
$$

Considering (5.34), and in light of Lyapunov theorem [23], one can conclude that the overall system (the communication network and the control gains) is globally ultimately stable. This completes the proof.

Corollary 1. The maximum bound on $y_{l 2}(t)$ in steady state is given by

$$
\left|y_{l 2}(t)\right| \leq \frac{\sqrt{N}+1}{2 a}
$$


where $l \in \mathbf{N}$. Furthermore, the bound on the overall performance of the system in steady state (5.29) is

$$
\sum_{i=1}^{N}\left|y_{i 2}(t)\right| \leq \frac{N}{a}
$$

Proof: For stability of the system (5.29), the derivative of the Lyapunov function as given by (5.34) has to be negative. In other words, to find the region of attraction, the trajectories $y_{i 1}(t), y_{i 2}(t), k_{i 1}(t)$ and $k_{i 2}(t)$ must be such that the following inequality holds

$$
\dot{V} \leq 0
$$

for all $i \in \mathbf{N}$. In this region of attraction, the maximum error magnitude for the $l$-th $\mathrm{AT}\left(y_{l 2}(t)\right)$ is resulted when $y_{i 1}(t)=0, k_{i 1}(t)=0, k_{i 2}(t)=0$, for all $i \in \mathrm{N}$, and $y_{i 2}=0$, for all $i \neq l$, i.e., $\left|y_{l 2}(t)\right| \leq \frac{\sqrt{N}+1}{2 a}$.

In addition, to find the bound on the overall performance $\sum_{i=1}^{N}\left|y_{i 2}\right|$, set $y_{i 1}(t)=0, k_{i 1}(t)=0, k_{i 2}(t)=0$, for all $i \in \mathbf{N}$. Then, using (5.37) one arrives at the following inequality

$$
\sum_{i=1}^{N}\left(\sqrt{a} y_{i 2}(t)-\frac{U(t)}{2 \sqrt{a}}\right)^{2} \leq \frac{U(t)^{2}}{4 a} N
$$

This bound can be simplified as

$$
\sum_{i=1}^{N}\left|y_{i 2}(t)\right| \leq \frac{N}{a}
$$

Remark 6. According to Corollary 1, in steady state, the upper bound on the discrepancy between the nominal rate $\bar{x}_{i 2}$ and the actual $A T$ rate $x_{i 2}$ for each $A T$ is

equal to $\frac{\sqrt{N}+1}{2 a}$. This implies that the upper bound on said discrepancy is inversely proportional to the control parameter a.

\subsection{Controller Design in Presence of Time-Delay}

It is now desired to investigate the impact of transmission delay on stability, and revisit the controller design proposed in the preceding section accordingly. Let the 


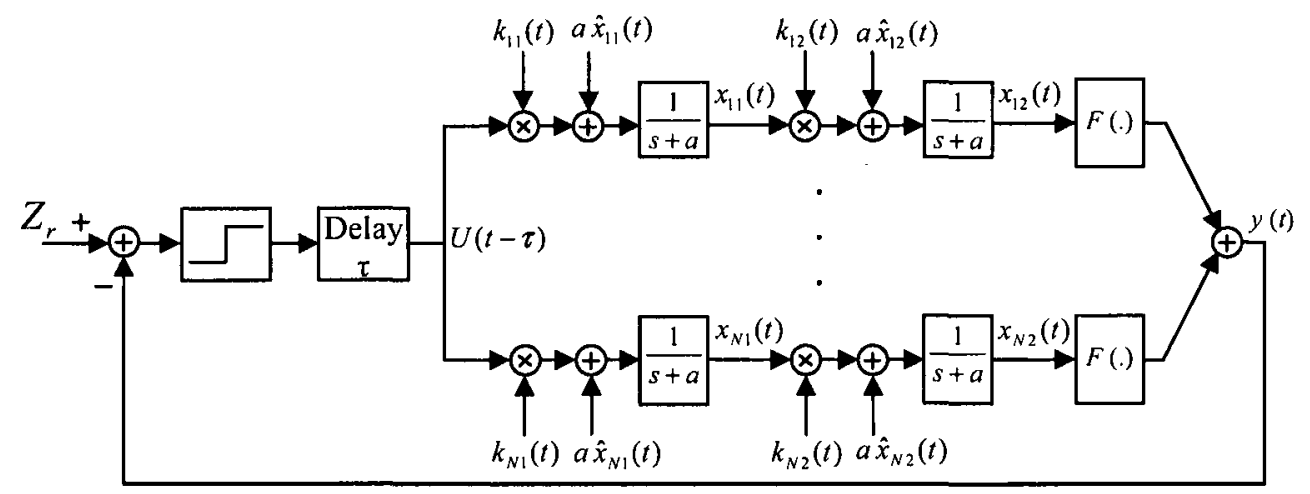

Figure 5.5: IS-856 uplink rate controller block diagram with the control low (5.40).

time-delay in the forward path be denoted by $\tau$, as shown in Fig. 5.5. In other words, AN transmits the signal $U(t)$ to ATs, and the ATs receive it after some delay. In terms of control operation, the input to the adaptive controller in this case is $U(t-\tau)$. The state space representation of the system with time delay is given by

$$
\begin{aligned}
{\left[\begin{array}{c}
\dot{x}_{11}(t) \\
\dot{x}_{12}(t) \\
\vdots \\
\dot{x}_{N 1}(t) \\
\dot{x}_{N 2}(t)
\end{array}\right]=} & {\left[\begin{array}{ccccc}
-a & 0 & \cdots & 0 & 0 \\
k_{12}(t) & -a & \cdots & 0 & 0 \\
\vdots & & \ddots & & \vdots \\
0 & 0 & \cdots & -a & 0 \\
0 & 0 & \cdots & k_{N 2}(t) & -a
\end{array}\right]\left[\begin{array}{c}
x_{11}(t) \\
x_{12}(t) \\
\vdots \\
x_{N 1}(t) \\
x_{N 2}(t)
\end{array}\right] } \\
& +\left[\begin{array}{c}
a \bar{x}_{11}(t)+k_{11}(t) U(t-\tau) \\
a \hat{x}_{12}(t) \\
\vdots \\
a \bar{x}_{N 1}(t)+k_{N 1}(t) U(t-\tau) \\
a \hat{x}_{N 2}(t)
\end{array}\right]
\end{aligned}
$$

and

$$
\dot{\hat{x}}_{i 2}(t)=U(t-\tau)
$$

Similar to the preceding section, the objective here is to ensure that $x_{i 1}(t)$ and $x_{12}(t)$ are regulated to a satisfactorily small neighborhood of the ideal nominal 
values 0 and $\bar{x}_{i 2}(t)$, respectively, where $\dot{\bar{x}}_{i 2}(t)=u(t)$. It is to be noted that the controller cannot generate $\bar{x}_{i 2}(t)$ because the signal $U(t)$ is not available to ATs. Instead, the controller will be using $U(t-\tau)$ in order to generate the signal $\hat{x}_{i 2}$, i.e. $\dot{\hat{x}}_{i 2}=U(t-\tau)$. By defining

$$
\begin{aligned}
& y_{i 1}(t)=x_{i 1}(t)-\bar{x}_{i 1}(t) \\
& y_{i 2}(t)=x_{i 2}(t)-\bar{x}_{i 2}(t)
\end{aligned}
$$

the state space representation (5.40) in the new coordination can be rewritten as follows

$$
\dot{Y}(t)=A(t) Y(t)+\hat{B}(t)
$$

where

$$
\hat{B}(t)=\left[\begin{array}{c}
k_{11}(t) U(t-\tau) \\
a\left(\hat{x}_{12}(t)-\bar{x}_{12}(t)\right)-U(t) \\
\vdots \\
k_{N 1}(t) U(t-\tau) \\
a\left(\hat{x}_{N 2}(t)-\bar{x}_{N 2}(t)\right)-U(t)
\end{array}\right]
$$

Using (5.41), the differential equations governing the adaptive gains can be written analogously to $(5.31)$ to obtain

$$
\begin{aligned}
& \dot{k}_{i 1}(t)=-a k_{i 1}(t)-U(t-\tau) y_{i 1}(t) \\
& \dot{k}_{i 2}(t)=-a k_{i 2}(t)-y_{i 1}(t)\left(y_{i 2}(t)+\bar{x}_{i 2}(t)-\hat{x}_{i 2}(t)\right)
\end{aligned}
$$

for all $i \in\{1, \ldots, N\}$.

The following theorem provides a sufficient condition for the stability of the system (5.42) in terms of the control parameter $a$.

Theorem 3. The state of the system (5.42) and the time-varying coefficients (5.44) are globally ultimately stable in the sense of Lyapunov if $a>\tau$. 
Proof: Choose the same Lyapunov function as (5.8). Differentiating (5.8) along the state trajectory of the system (5.42) yields

$$
\begin{aligned}
\dot{V}(t)= & -a y_{11}^{2}(t)+k_{11}(t) U(t-\tau) y_{11}(t)+k_{12}(t) y_{11}(t) y_{12}(t) \\
& -a y_{12}^{2}(t)+a y_{12}(t)\left(\hat{x}_{12}-\bar{x}_{12}\right)-U(t) y_{12}(t) \cdots \\
& -a y_{N 1}^{2}(t)+k_{N 1}(t) U(t-\tau) y_{N 1}(t) \\
& +k_{N 2}(t) y_{N 1}(t) y_{N 2}(t)-a y_{N 2}^{2}(t)+a y_{N 2}(t)\left(\hat{x}_{N 2}-\bar{x}_{N 2}\right) \\
& -U(t) y_{N 2}(t)+\sum_{i=1}^{N} \dot{k}_{i 1}(t) k_{i 1}(t)+\sum_{i=1}^{N} \dot{k}_{i 2}(t) k_{i 2}(t)
\end{aligned}
$$

Now, substituting (5.44) in (5.45), one will arrive at

$$
\begin{aligned}
\dot{V}(t)= & -a y_{11}^{2}(t)-a y_{12}^{2}(t)-a k_{11}^{2}(t)-a k_{12}^{2}(t) \\
& +a y_{12}(t)\left(\hat{x}_{12}-\bar{x}_{12}\right)+k_{12}(t) y_{11}(t)\left(\hat{x}_{12}-\bar{x}_{12}\right) \\
& -U(t) y_{12}(t) \cdots-a y_{N 1}^{2}(t)-a y_{N 2}^{2}(t)-a k_{N 1}^{2}(t) \\
& -a k_{N 2}^{2}(t)+a y_{N 2}(t)\left(\hat{x}_{N 2}-\bar{x}_{N 2}\right) \\
& +k_{N 2}(t) y_{N 1}(t)\left(\hat{x}_{N 2}-\bar{x}_{N 2}\right)-U(t) y_{N 2}(t)
\end{aligned}
$$

Note that

$$
\left|\hat{x}_{i 2}-\bar{x}_{i 2}\right|=\left|\int_{0}^{t_{0}} U(t-\tau)-\int_{0}^{t_{0}} U(t)\right| \leq 2 \tau
$$

for all $t_{0}>0$. Therefore, the derivative of the Lyapunov function in (5.46) can be 
rewritten as follows

$$
\begin{aligned}
\dot{V}(t) \leq & -a y_{11}^{2}(t)-a y_{12}^{2}(t)-a k_{11}^{2}(t)-a k_{12}^{2}(t)+2 a\left|y_{12}(t)\right| \tau \\
& +\left|k_{12}(t)\right|\left|y_{11}(t)\right| 2 \tau-U(t) y_{12}(t) \cdots-a y_{N 1}^{2}(t) \\
& -a y_{N 2}^{2}(t)-a k_{N 1}^{2}(t)-a k_{N 2}^{2}(t)+2 a\left|y_{N 2}(t)\right| \tau \\
& +2\left|k_{N 2}(t)\right|\left|y_{N 1}(t)\right| \tau-U(t) y_{N 2}(t) \\
= & -a k_{11}^{2}(t)-\left(\sqrt{a}\left|y_{12}(t)\right|-\frac{2 a \tau-U(t)}{2 \sqrt{a})^{2}}\right. \\
& -\left(\sqrt{a}\left|y_{11}(t)\right|-\frac{\left|k_{12}(t)\right| \tau}{\sqrt{a}}\right)^{2}+\frac{(2 a \tau-U(t))^{2}}{4 a} \\
& -k_{12}^{2}(t)\left(a-\frac{\tau^{2}}{a}\right)+\cdots \\
& -a k_{N 1}^{2}(t)-\left(\sqrt{a}\left|y_{N 2}(t)\right|-\frac{2 a \tau-U(t)}{2 \sqrt{a}}\right)^{2} \\
& -\left(\sqrt{a}\left|y_{N 1}(t)\right|-\frac{\left|k_{N 2}(t)\right| \tau}{\sqrt{a}}\right)^{2}+\frac{(2 a \tau-U(t))^{2}}{4 a} \\
& -k_{N 2}^{2}(t)\left(a-\frac{\tau^{2}}{a}\right)
\end{aligned}
$$

Since $a>\tau$, it can be concluded from Lyapunov theorem [16], [18] that the system is stable and all state variables and control parameters remain bounded. This completes the proof.

The following corollary gives an upper bound for each state variable and for the overall performance of the system.

Corollary 2. The maximum bound on each $y_{i 2}(t)$ in steady state is given by

$$
\left|y_{i 2}(t)\right| \leq \frac{(2 a \tau+1)}{2 a}(1+\sqrt{N})
$$

In addition, the bound on the sum of state variables in steady state is

$$
\sum_{i=1}^{N}\left|y_{i 2}(t)\right| \leq 2 \tau N+\frac{N}{a}
$$

Proof: The proof is similar to the one given for Corollary 1, and is ommitted due to space restrictions. 
Remark 7. Considering the stability condition $a>\tau$ in Theorem 2, in steady state, the maximum bounds on the state variables and the overall performance of the system can be obtained as follows

$$
\begin{gathered}
\max \left|y_{i 2}(t)\right|=\left(\tau+\frac{1}{2 \tau}\right)(1+\sqrt{N}) \\
\max \sum_{i=1}^{N}\left|y_{i 2}(t)\right|=2 \tau N+\frac{N}{\tau}
\end{gathered}
$$

\subsection{Stability Analysis for Varying Number of Users}

Assume now that the number of active ATs in the network is subject to change. Denote this number with $n=N(t) \leq \bar{N}$, where $\bar{N}$ is a known natural number, and let changes in $N(t)$ occur in the time sequence $\left\{t_{1}, t_{2}, t_{3}, \ldots\right\}$, where $t_{i}<t_{i+1}, \forall i \in \mathbb{N}$. This implies that the number of active ATs in $t_{k}^{-}$and $t_{k}^{+}(k \in \mathbb{N})$, are different. To maintain the balance in output $y(t)$, the $i$-th AT adjusts the parameter $\bar{x}_{i 2}$ continuously according to the number of active ATs available in the sector, denoted by $N(t)$, and the reference signal $Z_{r}$. To model changes in the number of users, (5.42) will be reformulated as an impulsive switched system [16], in such a way that any new activation or deactivation is translated to the jump from one subsystem to another. One can write

$$
\begin{cases}\dot{Y}(t)=A(t) Y(t)+B(t), & t \neq t_{k}, \forall k \in \mathbb{N} \\ Y(t)=f\left(Y\left(t^{-}\right), N(t)\right), & t=t_{k}, \forall k \in \mathbb{N}\end{cases}
$$

(note that the state vector $Y(t)$ is right-continuous). Here $f(\cdot)$ represents the jump in $Y(t)$ as a function of $Y\left(t^{-}\right)$and $N(t)$. For example, when a new activation or 
deactivation occurs in the $i$-th AT, the function $f(\cdot)$ is expressed as

$$
f\left(Y\left(t^{-}\right), N(t)\right)=Y\left(t^{-}\right)+\left[\begin{array}{c}
0 \\
\vdots \\
0 \\
\bar{x}_{i 2_{\text {old }}}-\bar{x}_{i 2_{\text {new }}} \\
0
\end{array}\right]
$$

In the above representation, for the case of activation $\bar{x}_{i 2_{\text {old }}}=0$ and $\bar{x}_{i 2_{\text {new }}}$ is set by the AN. For the case of deactivation, on the other hand, $\bar{x}_{i 2_{\text {new }}}$ is equal to zero and $\bar{x}_{i 2_{\text {new }}}$ is the last value calculated from (5.26) immediately before deactivation.

The objective now is to find a time interval for ATs, within which the network output is guaranteed to settle in a reasonably close neighborhood of the equilibrium point, when certain number of switches (instants of activation and deactivation of users) occur. To measure the performance, a strictly positive scalar $V_{d}$ is chosen, and it is desired that the Lyapunov function becomes smaller than $V_{d}$ after $S$ switches ( $S$ is an arbitrary natural number), and remains less than $V_{d}$ as time increases. It is to be noted that the maximum error in the system output is directly dependent on the value of $V_{d}$.

To proceed further, the following theorem is introduced.

Theorem 4. Denote the maximum value of $\bar{x}_{i 2}$ with $\Delta$, and let a strictly positive value $V_{d}$ and a natural number $S$ be given. Then, the maximum time it takes for the Lyapunov function to reduce to $V_{d}$ after the $S$-th change in the number of activations and deactivations, does not exceed a finite value $\bar{T}_{S}$ given below

$$
\bar{T}_{S}=\frac{1}{2\left(a-\tau-\frac{1}{2}\right)} \ln \left(\frac{2^{S} \max \left\{V\left(t_{0}\right), V_{d}\right\}+\sum_{i=0}^{S-1} 2^{S-i} \Delta^{2}}{V_{d}}\right)
$$

Proof: If the initial value of the Lyapunov function $V\left(t_{0}\right)$ is greater than the desired upper-bound $V_{d}$, then the maximum jump in the Lyapunov function (5.8) at 
the first switching instant $t_{1}$ can be calculated as

$$
V\left(t_{1}^{+}\right) \leq 2 V\left(t_{1}^{-}\right)+2 \Delta^{2}
$$

which after $S$ switches leads to the following inequality

$$
V\left(t_{S}^{+}\right) \leq 2^{S} V\left(t_{1}^{-}\right)+\sum_{i=0}^{S-1} 2^{S-i} \Delta^{2}
$$

The derivative of the Lyapunov function in (5.47) can be shown to satisfy the following inequality

$$
\begin{aligned}
\dot{V}(t) \leq & -\left(a-\tau-\frac{1}{2}\right) \sum_{i=1}^{N} y_{i 1}^{2}(t)-\left(a-\tau-\frac{1}{2}\right) \sum_{i=1}^{N} y_{i 2}^{2}(t) \\
& -\left(a-\tau-\frac{1}{2}\right) \sum_{i=1}^{N} k_{i 1}^{2}(t)-\left(a-\tau-\frac{1}{2}\right) \sum_{i=1}^{N} k_{i 2}^{2}(t) \\
& +N \frac{(1+2 a \tau)^{2}}{2}
\end{aligned}
$$

Let the time it takes for the Lyapunov function to decay down to $V_{d}$ following the $S$-th switch be denoted by $T_{S}$. Using (5.57), one can conclude that the inequality given below holds

$$
\mathrm{e}^{-2\left(a-\tau-\frac{1}{2}\right) T_{S}} V\left(t_{S}^{+}\right) \leq V_{d}
$$

and subsequently, the time $\bar{T}_{S}$ defined in the theorem (5.54) is given by

$$
\bar{T}_{S}=\frac{1}{2\left(a-\tau-\frac{1}{2}\right)}\left[\ln \left(2^{S} V\left(t_{1}^{-}\right)+\sum_{i=0}^{S-1} 2^{S-i} \Delta^{2}\right)-\ln \left(V_{d}\right)\right]
$$

which can be simplified as

$$
\bar{T}_{S}=\frac{1}{2\left(a-\tau-\frac{1}{2}\right)} \ln \left(\frac{2^{S} V\left(t_{0}\right)+\sum_{i=0}^{S-1} 2^{S-i} \Delta^{2}}{V_{d}}\right)
$$

On the other hand, a conservative estimate for the Lyapunov function for the case when $V_{d}>V\left(t_{0}\right)$ would be $V_{d}$, after $S$ switches. Therefore, one arrives at the following equality instead of (5.60) at in this case

$$
\bar{T}_{S}=\frac{1}{2\left(a-\tau-\frac{1}{2}\right)} \ln \left(\frac{2^{S} V_{d}+\sum_{i=0}^{S-1} 2^{S-i} \Delta^{2}}{V_{d}}\right)
$$


This completes the proof.

Remark 8. It follows immediately from Theorem 3 (by substituting $S=1$ in (5.54)) that if after any changes in the number of active users, no new activation or deactivation occurs in the network for a time interval given by

$$
\bar{T}_{1}=\frac{1}{2\left(a-\tau-\frac{1}{2}\right)} \ln \left(\frac{2 V_{d}+2 \Delta^{2}}{V_{d}}\right)
$$

then the Lyapunov function decays down to the prescribed value $V_{d}$, and stays smaller than that as time increases (it is to be noted that this is only a sufficient condition for the desirable decrease in the size of the Lyapunov function, which is closely related to the output performance).

\subsection{Equilibrium Point Adjustment: Long-Term Fairness Study}

Consider a long-term fairness strategy, under which subscription fees vary by the assigned rates. In other words, the customers can receive a higher data rate within the allowable range if they choose to pay a higher access fee, accordingly. It is to be noted, however, that just like the uniform rate-assignment strategy, in the fairness strategy also the overall interference of the network is to be regulated around $Z_{r}$.

To formulate fairness in the rate assignment, one can use an appropriately weighted function of RAB (instead of a simple integral implied by (5.26)) in the regulation problem. For example, by employing different weighting and bias coefficients for different users, one can introduce the following function:

$$
\bar{x}_{i 2}(t)=c_{i} \int_{0}^{t} U\left(t^{\prime}\right) d t^{\prime}+b_{i}
$$

where $c_{i}$ and $b_{i}$ are positive constants with known upper bounds $c_{i}<\psi$ and $b_{i}<\beta$, for all $i \in \mathbf{N}$ (the constants $\psi$ and $\beta$ depend on the number of users and the allowable 
deviation between different users' assigned rates). The minimum guaranteed rates for ATs can be set through the parameter $b_{i}$ for each AT, and the parameter $c_{i}$ can then be adjusted in accordance with the subscription contracts of different users.

It can be shown that

$$
\left|\hat{x}_{i 2}-\bar{x}_{i 2}\right|=\left|\psi \int_{0}^{t_{0}} U\left(t^{\prime}-\tau\right) d t^{\prime}-\psi \int_{0}^{t_{0}} U\left(t^{\prime}\right) d t^{\prime}\right| \leq 2 \tau \psi
$$

Now, for the case of fixed number of users, using an approach similar to the one in Theorem 3 and employing the same Lyapunov function as in (5.8), it can be concluded that the system is globally ultimately stable in the sence of Lyapunov if $a>\tau \psi$

$$
\begin{aligned}
\dot{V}(t) \leq & -\sum_{i=1}^{N} K_{i 1}^{2}(t)-\sum_{i=1}^{N}\left(\sqrt{a}\left|y_{i 2}(t)\right|-\frac{2 a \psi \tau-\psi U(t)}{2 \sqrt{a}}\right)^{2} \\
& -\sum_{i=1}^{N}\left(\sqrt{a}\left|y_{i 1}(t)\right|-\frac{\left|K_{i 2}(t)\right| \tau \psi}{\sqrt{a}}\right)^{2} \\
& -\sum_{i=1}^{N} K_{i 2}^{2}(t)\left(a-\frac{\tau^{2} \psi^{2}}{a}\right)+\sum_{i=1}^{N} \frac{(2 a \tau \psi-\psi U(t))^{2}}{4 a}
\end{aligned}
$$

Furthermore, the maximum bound on each $y_{i 2}(t)$ in steady state is given

$$
\left|y_{i 2}(t)\right| \leq \frac{\psi}{2 a}(1+\sqrt{N})(2 a \tau+1)
$$

The result in (5.65) is derived for the case of fixed number of users. In order to show the stability of the network for the case when the number of users is subject to change and to find the sufficient condition for stability (in terms of the time interval between the consecutive changes in the number of users), one can take an approach similar to the one in Theorem 4. To proceed further, a new bound can be obtained on the derivative of Lyapunov function as

$$
\begin{aligned}
\dot{V}(t) \leq & -\left(a-\psi \tau-\frac{1}{2}\right) \sum_{i=1}^{N} y_{i 1}^{2}(t)-\left(a-\psi \tau-\frac{1}{2}\right) \sum_{i=1}^{N} y_{i 2}^{2}(t) \\
& -\left(a-\psi \tau-\frac{1}{2}\right) \sum_{i=1}^{N} k_{i 1}^{2}(t)-\left(a-\psi \tau-\frac{1}{2}\right) \sum_{i=1}^{N} k_{i 2}^{2}(t) \\
& +N \frac{(\psi+2 a \psi \tau)^{2}}{2}
\end{aligned}
$$


Therefore, the maximum time it takes for the Lyapunov function to reduce to $V_{d}$ after the $S$-th change in the number of activations and deactivations, does not exceed a finite value $\bar{T}_{S}$ given below

$$
\bar{T}_{S}=\frac{1}{2\left(a-\psi \tau-\frac{1}{2}\right)} \ln \left(\frac{2^{S} \max \left\{V\left(t_{0}\right), V_{d}\right\}+\sum_{i=0}^{S-1} 2^{S-i} \Delta^{2}}{V_{d}}\right)
$$

Remark 9. The results obtained show that choosing a large value for the control parameter a would be advantageous in terms of the maximum bound on the output (as can be seen from (5.35), (5.36), (5.48) and (5.49)), in terms of the minimum required time for consecutive activation and deactivations (as can be seen from (5.62)), and also in terms of convergence time (as can be seen from (5.12)). However, a large a can have implications in terms of the implementation of the system in practice. A large a would increase the control bandwidth, which in turn increases its sensitivity to noise. Furthermore, a large a can cause saturation due to the increase in the magnitude of some of the signals in the loop. This introduces a trade-off in the choice of the coefficient $a$ in the control law.

\subsection{Simulation Results}

In this section, a simulation scenario is considered where multiple ATs are available in a single cell. The reference $Z_{r}$ is set to 171.23 , which corresponds to the system capacity of $600 \mathrm{Kbps}$ [1]. The simulation is repeated for two different values of the parameter $a$ to demonstrate the effect of this parameter on the system response. Let the initial number of the users in the cell be $N(0)=20$, and note that this number changes at different points in time due to the new activations and deactivations. As a result, active ATs adjust their control parameters continuously (using the proposed adaptive control scheme). In these simulations, $V_{d}$ is set to 1 , which results in

$\bar{T}_{1}=500 \mathrm{msec}$ for $a=10$ and $\bar{T}_{1}=50 \mathrm{msec}$ for $a=100$, that are realistic values in practice. Furthermore, the delay in the network structure is assumed to be constant 
Table 5.1: Performance comparison for different methods and different values of the control parameter $a$.

\begin{tabular}{|c|c|}
\hline & Performance Index $\times 10^{4}$ \\
\hline First-order Approximation; $\mathrm{a}=10$ & 13.298 \\
\hline First-order Approximation; $\mathrm{a}=100$ & 13.274 \\
\hline Second-order Approximation; $\mathrm{a}=10$ & 4.373 \\
\hline Second-order Approximation; $\mathrm{a}=100$ & 4.196 \\
\hline Dynamic probabilistic Allocation; $\mathrm{a}=10$ & 1.085 \\
\hline Dynamic probabilistic Allocation; $\mathrm{a}=100$ & 0.912 \\
\hline
\end{tabular}

and equal to $13.36 \mathrm{msec}$, which is equivalent to 8 time slots. In addition, let the instants (in seconds) at which the number of active users changes be 10, 15, 20, 25, $40,50,65,80,85,90,95,100$. It is to be noted that in all simulations the exact mapping $F(\cdot)$ is used to find the network output.

In Fig. 5.6(a), the network output $y(t)$ with first-order approximation (5.15) for the mapping function is depicted for $a=10$ along with its moving average. In Fig. 5.6(b), analogous results are shown for $a=100$. The number of active users in the network (which is considered in all simulations) is drawn versus time in Fig. 5.6(c). Note that the smallest time gap between two consecutive changes in the number of users in this example is $5 \mathrm{sec}$, which is greater than $\bar{T}_{1}=500 \mathrm{msec}$ introduced in Remark 7. Thus, it is guaranteed that the desired performance (in terms of the final magnitude of the Lyapunov function) will be achieved. However, since the time limit obtained is only a sufficient condition, the network may perform desirably for a more frequent change of active users as well. The results obtained by using second-order approximation for the mapping function are shown analogously in Fig. 5.7. In Fig. 5.8, the same scenario is considered for the case when the number of users is unknown to the ATs, and the probabilistic rate selection method is adopted. For each one of the scenarios discussed above, the performance index $\int_{0}^{T} e^{2}(t) d t$ is computed and the results are provided in Table 1. 
(a)

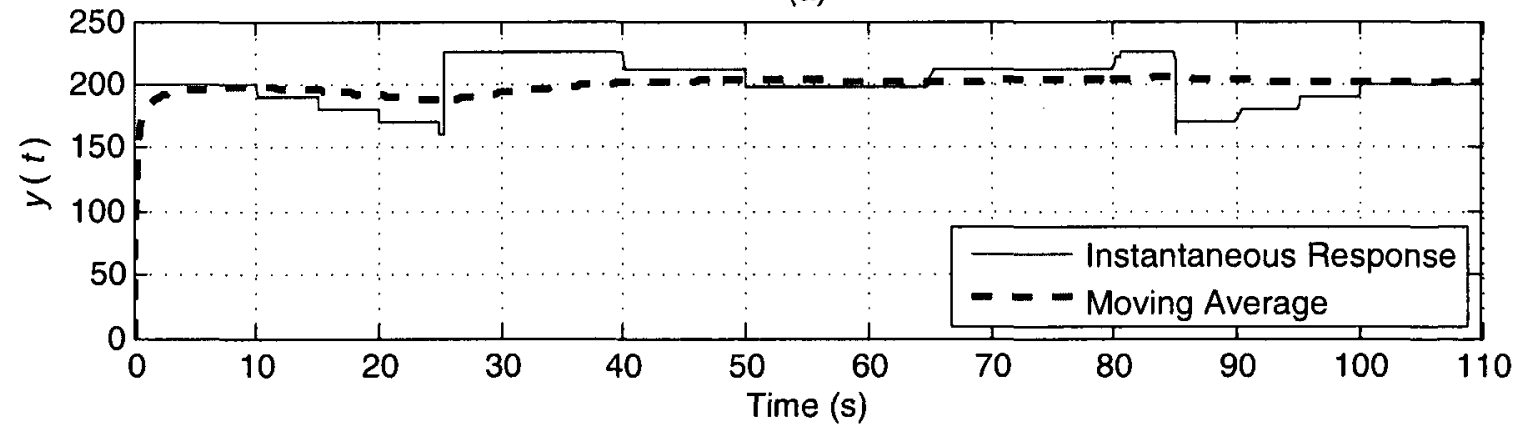

(b)

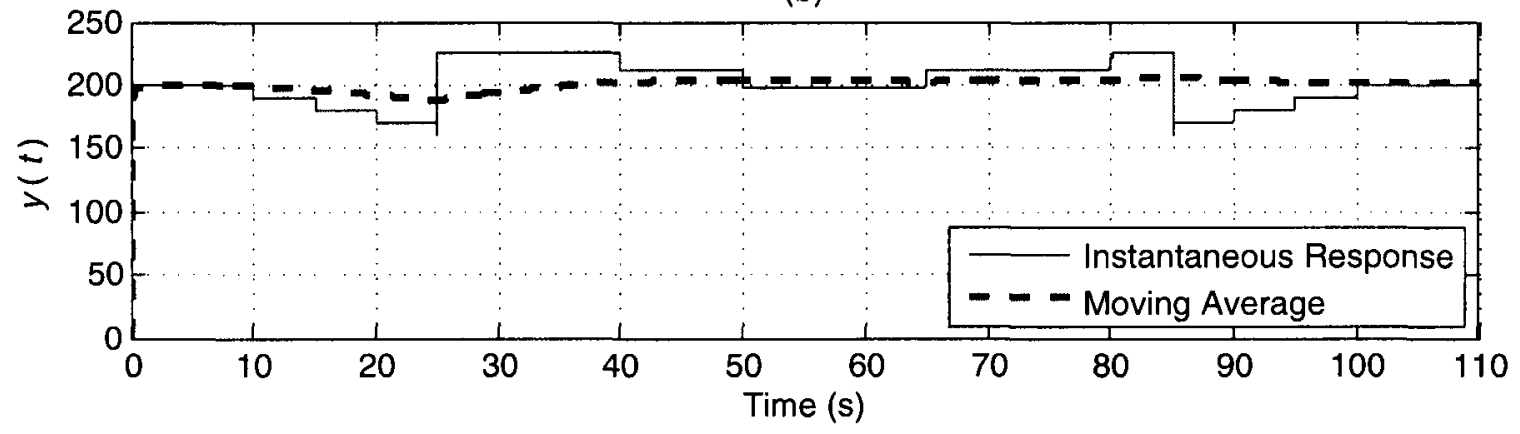

(c)

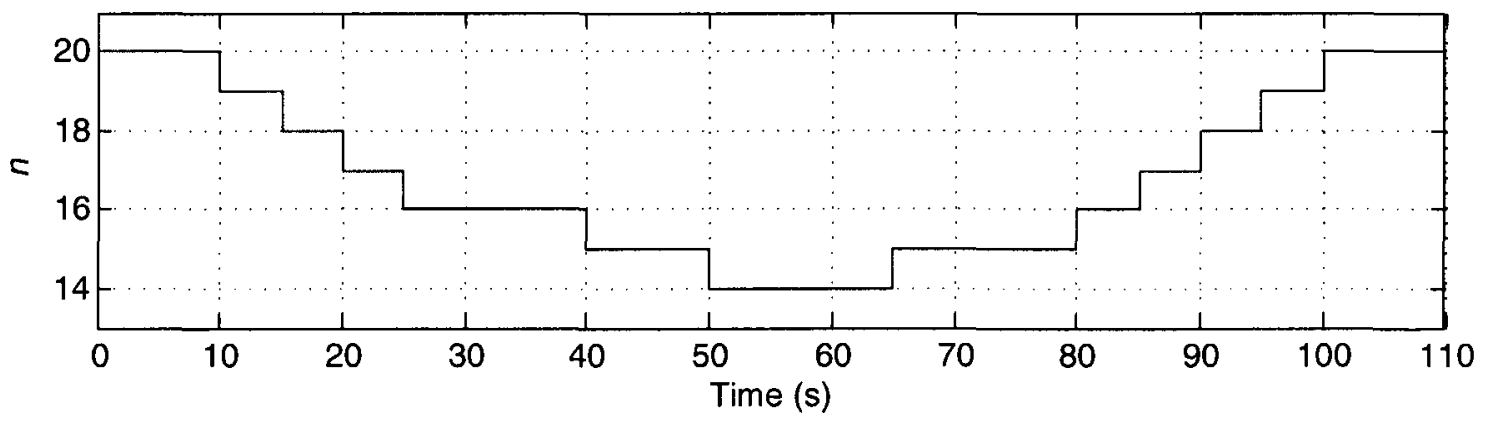

Figure 5.6: The network output $y(t)$ resulted from using first-order approximation (a) $a=10$; (b) $a=100$. (c) The number of available ATs considered in the simulations at different times. 
(a)

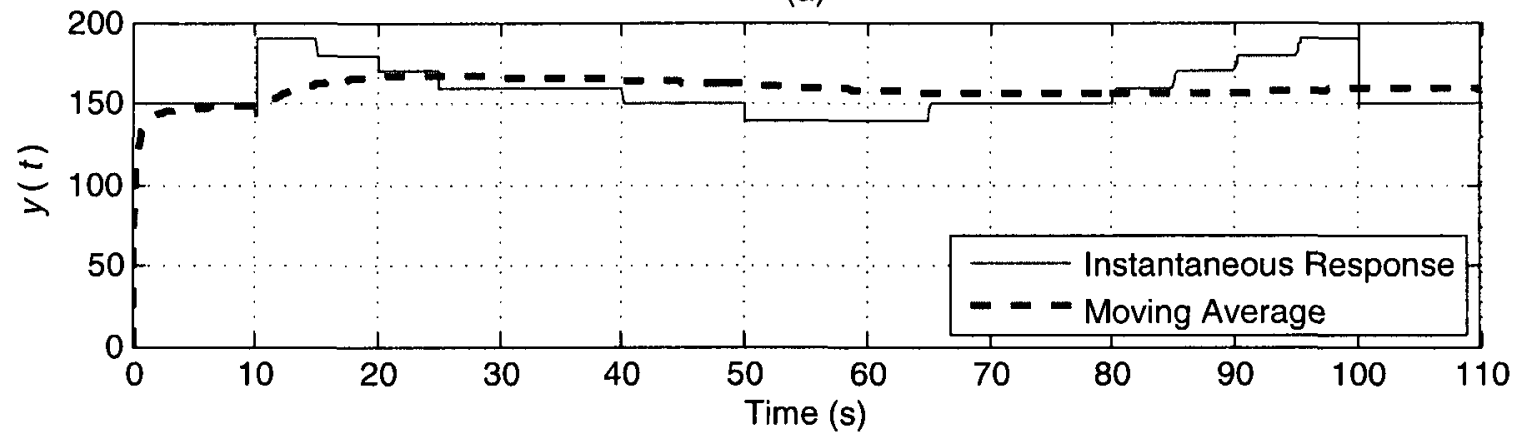

(b)

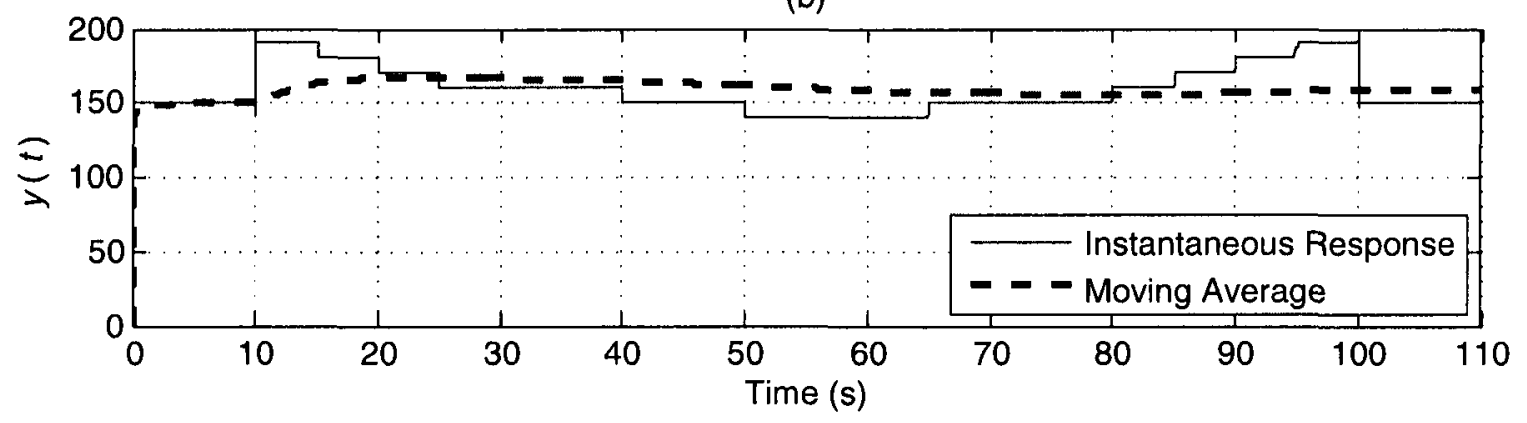

(c)

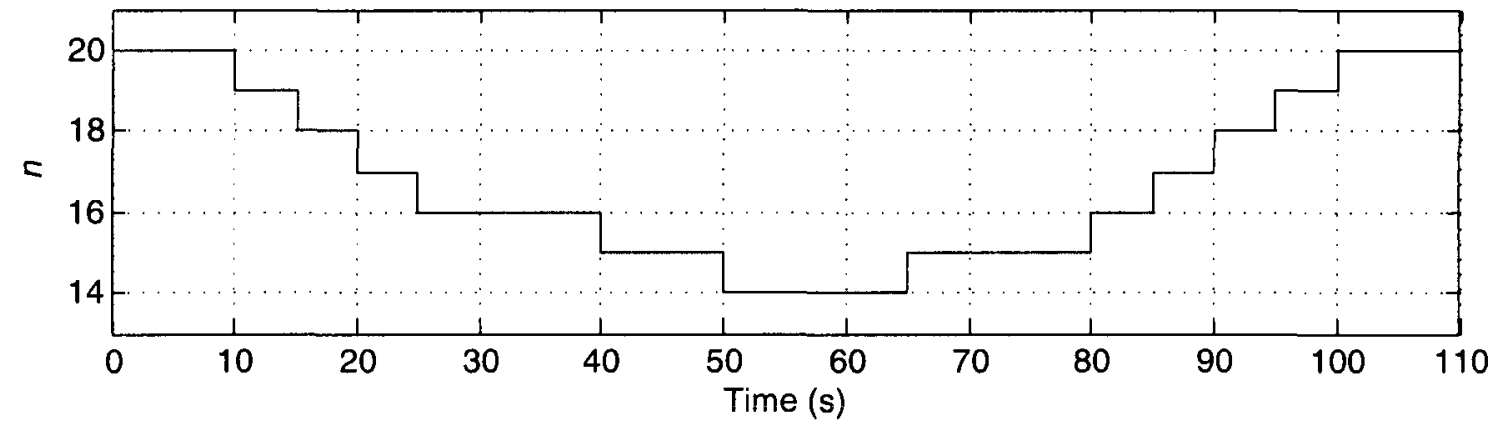

Figure 5.7: The network output $y(t)$ resulted from using second-order approximation (a) $a=10$; (b) $a=100$. (c) The number of available ATs considered in the simulations at different times. 
(a)

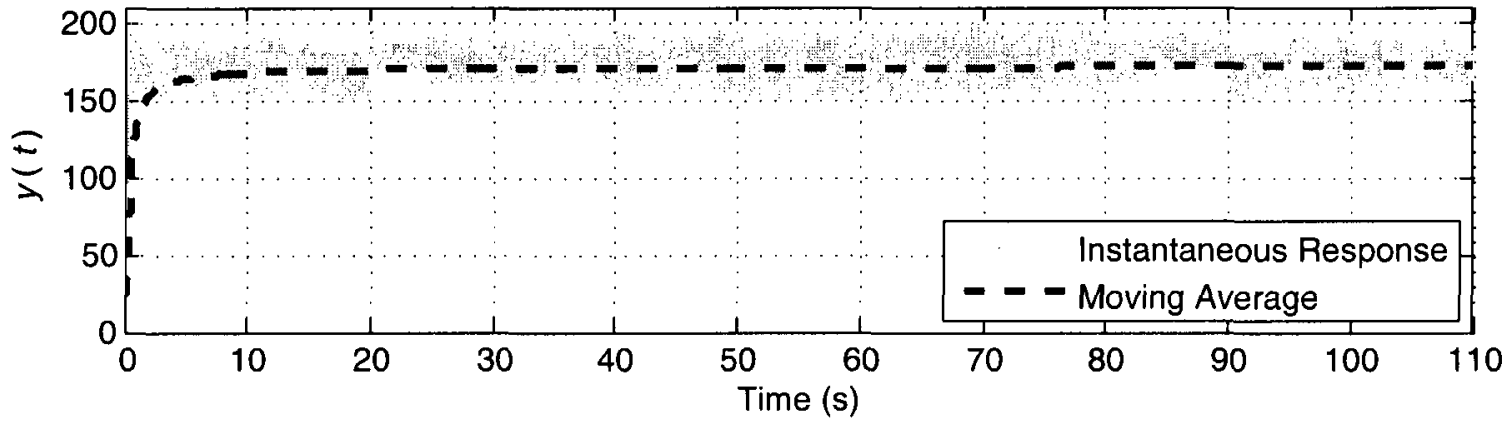

(b)

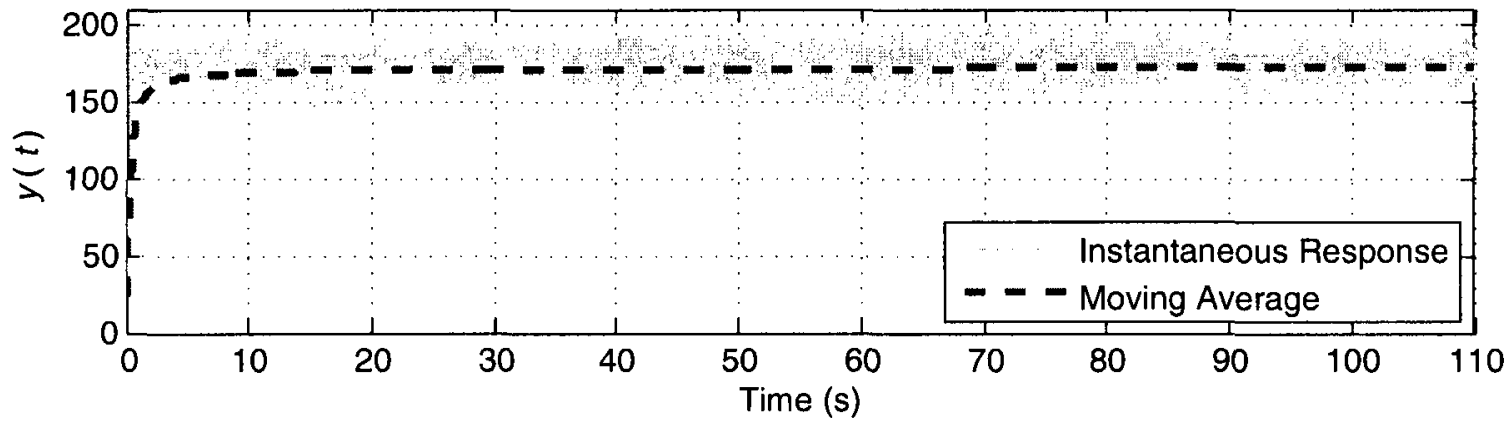

(c)

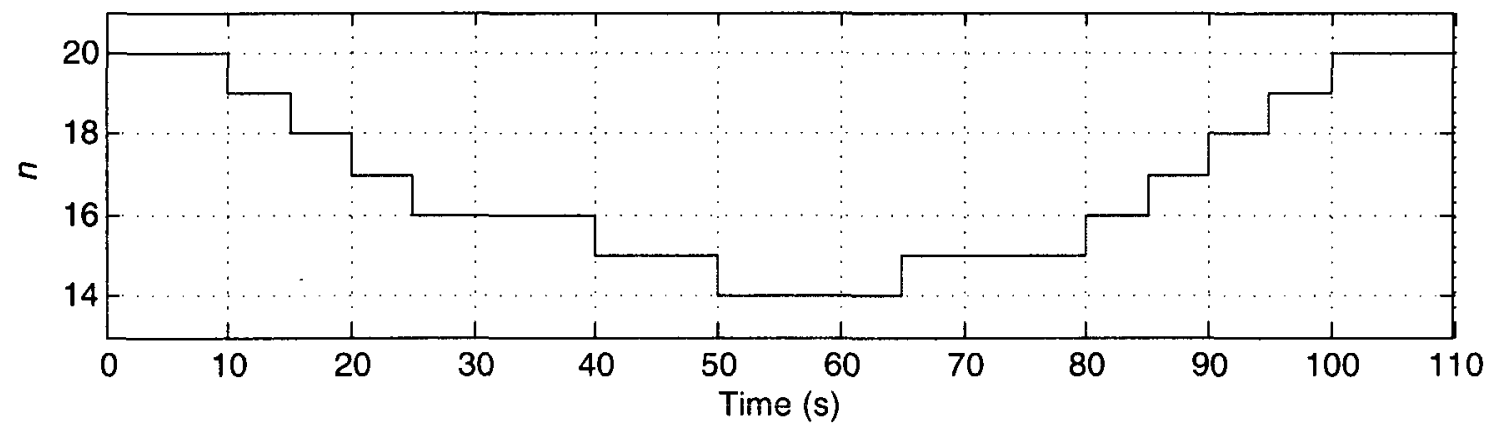

Figure 5.8: The network output $y(t)$ resulted from using dynamic probability rate allocation method (a) $a=10$; (b) $a=100$. (c) The number of available ATs considered in the simulations at different times. 
In all Figs. 5.6-5.8, the network output $y(t)$ is regulated around the reference signal $Z_{r}$. By Comparing the instantaneous and average outputs of Fig. 5.6 with those of Figs. 5.7, 5.8, and from the performance indices provided in Table 5.1, it can be deduced that the network output is better regulated when the dynamic probability rate allocation method is utilized, and a larger value for the control parameter $a$ is chosen.

A simulation scenario is also considered, in which the parameters of two active ATs are set to

$$
\begin{aligned}
& b_{1}=b_{2}=30 \\
& c_{1}=c_{2}=5
\end{aligned}
$$

while the control parameters of all other ATs are given by

$$
\begin{aligned}
b_{i} & =0 \\
c_{i} & =100
\end{aligned}
$$

for $i=3,4, \ldots, N$. In this case, the network output for an arbitrary always-active AT is depicted in Fig. 5.9 (a) while the network output for the first and second ATs is depicted in Fig. 5.9 (b). The number of active users in the network (which is simliar to the previous simulations) is drawn versus time in Fig. 5.9 (c). From this figure, one can compare the effect of network load as well as the activation and deactivation of users on the output for these two particular ATs. The first and second ATs which have higher subscription fees (which is formulated by a large $b_{i}$ and small $c_{i}$ ) have higher transmission rates compared to other users (with a small $b_{i}$ and large $c_{i}$ ). Moreover, the sensitivity of these two users to the network load is less than that of the other users, as it can be seen at the instants of change in the number of active users, while the outputs of other ATs highly depend on these time instants. 
(a)

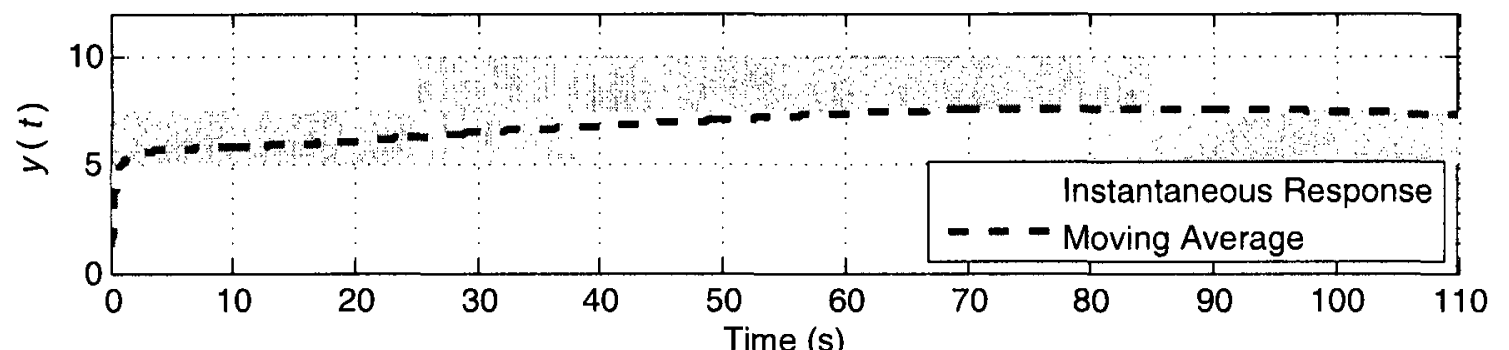

(b)

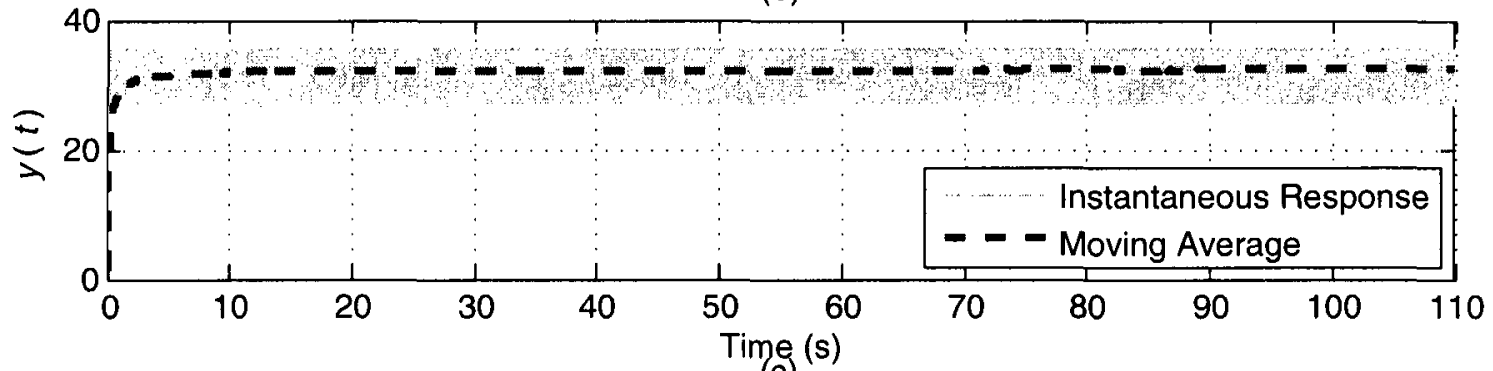

(c)

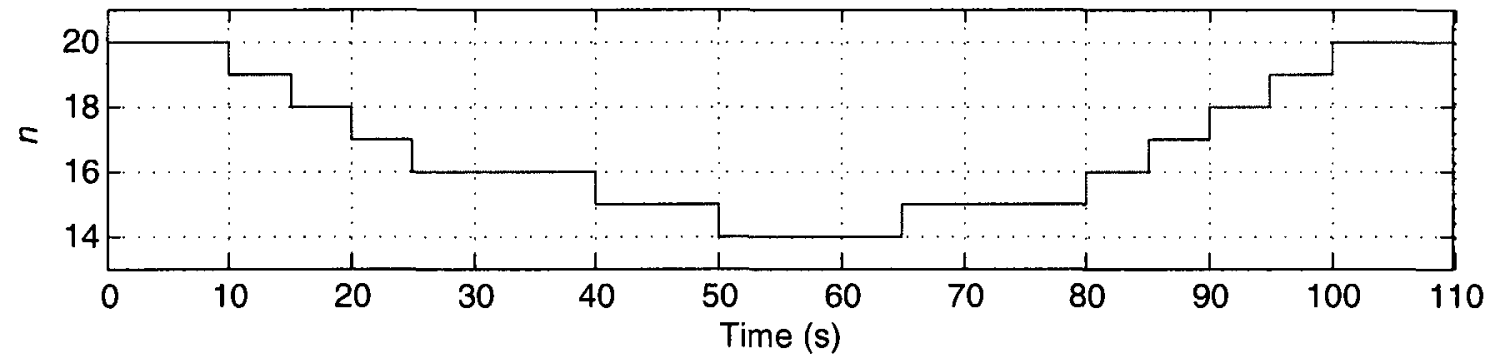

Figure 5.9: The output $y_{i}(t)$ resulted from using dynamic probability rate allocation method; (a) the ATs with the control parameters given by (5.70); (b) the ATs with the control parameters given by (5.69). (c) The number of available ATs considered in the simulations at different times. 


\section{Chapter 6}

\section{Conclusions and Future Work}

In this thesis, the problem of rate assignment in wireless communication networks is investigated in the control theoretic framework. The IS-856 uplink is modeled for one user and also for the entire network. Effective control methods such as Lyapunov analysis and describing function are then employed to tackle important problems concerning rate assignment in the network.

The control signal transmitted from the base station to the users is binary, which is formulated as an on-off nonlinear element in the context of control. A simple delay is considered as the model of each user, and a first order approximation is made for the mapping of the feasible rate set to the feasible $T 2 P$ set; the describing function technique is used subsequently to analyze any possible oscillation in the output of the system in the framework of limit cycles. It is shown that using a two-bit control signal (instead of one-bit) to quantize the error can significantly improve the performance of the system output. This, however, introduces a trade-off between allocating as many bits as possible to the transmitted data, and improving the system performance.

As a first step in control design, a model is developed for IS-856 uplink. Different approximations are introduced for the mapping of the feasible rate set in this 
model. An adaptive control scheme is then designed, which uses the current state of the network to adjust the control parameters accordingly. It is shown that using a dynamic rate selection in the mapping of feasible rate set can enhance the overall control performance in the network.

There are certain issues in a practical environment which need to be incorporated in the network model for the control design. For example, the number of active users in the network changes by time. Furthermore, the control signal in the forward path is subject to delay. However, in the design procedure, it is first assumed that the number of users in the network is fixed and that there is no delay in the control loop. The stability of the resulting closed-loop network is analyzed thoroughly using an effective control tool, namely Lyapunov technique. The results obtained are then extended to the case of nonzero delay, and a sufficient condition for stability is obtained in terms of the magnitude of delay. In addition, the varying number of active users in the network is formulated as a switched system. A sufficient condition is obtained subsequently for the stability of the network in terms of the speed of activation/deactivation of the users. More precisely, a lower-bound on the time-interval between consecutive changes in the number of active users is obtained to guarantee the stability of the network. Simulations show that the resultant lower-bound is not too conservative; in other words, the sufficient condition obtained is close to necessity in a practical setting. A novel technique is also introduced to attain an upper-bound on the magnitude of the regulation error in the network.

The proposed adaptive controller provides an efficient rate assignment mechanism for the users in the wireless network. However, it is often desirable to develop a fairness strategy with a prescribed non-uniform rate assignment feature. In order to take fairness in rate assignment into account, a weighted function of RAB is considered in the regulation problem to prioritize users according to their subscription 
fees. Using this formulation, an approach similar to the one used in the previous case (uniform rate assignment) can be taken to solve the fairness problem.

While the techniques provided in this thesis prove effective in analysis of rate assignment and control design for wireless networks, certain issues can be further investigated as the continuation of the present work. First of all, a more precise model can be used for the network, by considering channel fading and mobility, noise, variation of network parameters, etc. into account. One can also consider the undesirable effect of imperfect power control in the network. The interferences caused by those users that are not in soft hand-off with the base station can be incorporated in the model for a better accuracy, as another direction for future research. Finally, the adaptive controller designed in this thesis consisted of a fixed first-order transfer function (in addition to the state-dependent parameters). It would be interesting to consider more complex control laws instead of this simple first-order unit to achieve a higher performance. 


\section{Bibliography}

[1] 1xEV: 1xEVolution IS-856 TIA/EIA Standard, airlink overview, available at http://www.cdg.org November 2001, Revision 7.2.

[2] 3G - CDMA2000 1xEV-DO Technologies, available at http://www.cdg.org (2009).

[3] International Telecommunication Homepage, available at http://www.itu.net (2009).

[4] P. Bender, P. Black, M. Grob, R. Padovani, N. Sindhushayana, and A.Viterbi, "CDMA/HDR: A bandwidth efficient high-speed wireless data service for nomadic users," IEEE Communications Magazine, pp. 70-77, July 2000.

[5] Q. Wu and E. Esteves, "The cdma2000 high rate packet data system," Qualcomm white paper, 2002.

[6] J.-J. E. Slotine and W. Li, Applied Nonlinear Control, Prentice-Hall International Inc., 1991.

[7] CDMA2000 High Rate Packet Data Air Interface Specification, available at http://www.3gpp2.org (2005).

[8] A. J. Viterbi, CDMA: Principles of Spread Spectrum Communication, Addison-Wesley Wireless Communication, 1995. 
[9] K. Il Kim, Handbook of CDMA System Design, Engineering and Optimization, Prentice-Hall, 2000.

[10] P. Tinnakornsrisuphap and C. Lott, "On the fairness and stability of the reverse-link MAC layer in CDMA2000 1xEV-DO," in Proceedings of IEEE International Conference on Communications, vol. 1, pp. 144-148, Paris, France, June 2004.

[11] R. Yim, O.-S. Shin, and V. Tarokh, "Reverse-link rate control algorithms with fairness guarantees for CDMA systems," IEEE Transactions on Wireless Communications, vol. 6, no. 4, pp. 1386-1397, April 2007.

[12] E. Esteves, "On the reverse link capacity of cdma2000 high rate packet data systems," in Proceedings of IEEE International Conference on Communications, vol. 3, pp. 1823-1828, New York, USA, May 2002.

[13] J. Price and T. Javidi, "Decentralized rate assignments in a multi-sector CDMA network," IEEE Transactions on Wireless Communications, vol. 5, no. 12, pp. 3537-3547, December 2006.

[14] H. Alasti and A. G. Aghdam, "Rate control in CDMA2000 IS-856 up-link: a control theoretic view," in Proceedings of American Control Conference, pp. 4888-4893, Minneapolis, USA, June 2006.

[15] D. Liberzon, Switching in Systems and Control, Birkhäuser Press, Boston, 2003.

[16] J. P. Hespanha, D. Liberzon, and A. R. Teel, "On input-to-state stability of impulsive systems," in Proceedings of $44^{\text {th }}$ IEEE Conference on Decision and Control, pp. 3992-3997, December, 2005.

[17] F. Kelly, "Charging and rate control for elastic traffic," European Transactions on Telecommunications, vol. 8, issue 1, pp. 33-37, January-February 1997. 
[18] F. Kelly, A. Maulloo, and D. Tan, "Rate control in communication networks: shadow prices, proportional fairness and stability," Journal of the Operational Research Society, pp. 237-252, March 1998.

[19] S. Chakravarty, R. Pankaj, and E. Esteves, "An algorithm for reverse traffic channel rate control for cdma2000 high rate packet data systems," in Proceedings of IEEE Global Telecommunications Conference, vol. 6, pp. 3733-3737, San Antonio, USA, November 2001.

[20] R. Padovani, "The application of spread spectrum to PCS has become a reality: reverse link performance of IS-95 based cellular systems," IEEE Personal Communications, issue 3, pp. 28-34, third quarter 1994.

[21] R. Attar and E. Esteves, "A reverse link outer-loop power control algorithm for cdma2000 1xEV systems," in Proceedings of IEEE International Conference on Communications, vol. 1, pp. 573-578, New York, USA, May 2002.

[22] K. Jalaleddini, K. Moezzi, A. G. Aghdam, M. Alasti, and V. Tarokh, "Controller design for rate assignment in wireless networks," in Proceedings of IEEE International Conference on Communications, Dresden, Germany, June 2009.

[23] H. K. Khalil, Nonlinear Systems, Prentice Hall, New Jersey, 2002.

[24] D. D. Bainov and P. S. Simeonov, Systems With Impulse Effect: Stability, Theory and Applications, Ellis Horwood, UK, 1989.

[25] E. K. P. Chong and S. H. Żak, An Introduction to Optimization, Wiley, USA, 2001.

[26] K. Moezzi, K. Jalaleddini, A. G. Aghdam, M. Alasti, and V. Tarokh, "An adaptive rate assignment strategy for CDMA2000 IS-856 subject to RAB delay," in Proceedings of IEEE Global Communications Conference, Honolulu, USA, December 2009 (to appear). 
[27] K. Jalaleddini, K. Moezzi, A. G. Aghdam, M. Alasti, and V. Tarokh, "Rate assignment in wireless networks: stability analysis and controller design," to be submitted to IEEE Transactions on Wireless Communications.

[28] A. Jalali, R. Padovani, and R. Panjkaj, "Data throughput of CDMA-HDR a high efficiency-high data rate personal communication wireless system," in Proceedings of IEEE 51 ${ }^{\text {st }}$ Vehicular Technology Conference, vol. 3, pp. 18541858, Tokyo, Japan, May 2000.

[29] H. Alasti, A. G. Aghdam, and M. Alasti, "An optimal rate assignment mechanism for multimedia transmission over the down-link of IS856," in Proceedings of $45^{\text {th }}$ IEEE Conference on Decision and Control, pp. 627-632, San Diego, USA, December 2006.

[30] A. Demers, S. Keshav, and S. Shenkar, "Analysis and simulation of a fair queueing algorithm," in Proceedings of SIGCOMM, vol. 19, pp. 1-12, Austin, USA, September 1989.

[31] A. K. Parekh and R. G. Gallager, "A generalized processor sharing approach to flow control in integrated services networks-the single node case," in Proceedings of IEEE INFOCOM, pp. 915-924, Florence, Italy, May 1992.

[32] C. E. Koksal, H. Kassab, and H. Balakrishnan, "An analysis of short-term fairness in wireless media access protocols," in Proceedings of ACM SIGMETRICS, pp. 118-119, Santa Clara, USA, June 2000.

[33] R. T. Derryberry, "1xEV-DV evaluation methodology" 3GPP2/TSGC.R1002, 3GPP2 technical specification group $C$.

[34] T. Alpcan, T. Basar, R. Srikant, and E. Altman, "CDMA uplink power control as a noncooperative game," in Proceedings of $40^{\text {th }}$ IEEE Conference on Decision and Control, pp. 197-202, Orlando, USA, December 2001. 
[35] R. D. Yates, "A framework for uplink power control in cellular radio systems," IEEE Journal on Selected Areas in Communications, vol. 13, pp. 1341-1347, September 1995.

[36] M. Fan, D. Ghosh, N. Bhushan, R. Attar, C. Lott, and J. Au, "On the reverse link performance of cdma2000 1xEV-DO revision A system," in Proceedings of IEEE International Conference on Communications, pp. 2244-2250, Seoul, South Korea, May 2005.

[37] M. G. Jansen and R. Prasad, "Capacity, throughput, and delay analysis of a cellular DS CDMA system with imperfect power control and imperfect sectorization," IEEE Transactions on Vehicular Technology, vol. 44, pp. 67-75, February 1995.

[38] J. Price and T. Javidi, "Cross-Layer (MAC and transport) optimal rate assignment in CDMA-based wireless broadband networks," in Conference Record of the Thirty-Eighth Asilomar Conference on Signals, Systems and Computers, pp. 1044-1048, Pacific Grove, USA, November 2004.

[39] J. Price and T. Javidi, "Decentralized rate assignments in a multi-sector CDMA network," IEEE Transactions on Wireless Communications, vol. 5, no. 12, pp. 3537-3547, December 2006.

[40] R. Yim, O.-S. Shin, and V. Tarokh, "Distributed delay-aware rate control algorithm for the reverse-link of CDMA systems," in Proceedings of IEEE International Conference on Communications, pp. 4363-4368, Istanbul, Turkey, June 2006 .

[41] B. A. Francis, and W. M. Wonham, "The internal model principle for linear multivariable regulators," Applied Mathematics and Optimization, vol. 2, pp. 170-194, June 1975. 
[42] R. C. Dorf, and R. H. Bishop, Modern Control Systems, Prentice-Hall, 2007. 